\title{
MODIFIED SCATTERING FOR THE CUBIC SCHRÖDINGER EQUATION ON PRODUCT SPACES AND APPLICATIONS
}

\author{
ZAHER HANI $^{1}$, BENOIT PAUSADER ${ }^{2}$, NIKOLAY TZVETKOV ${ }^{3}$ and \\ NICOLA VISCIGLIA ${ }^{4}$ \\ ${ }^{1}$ School of Mathematics, Georgia Institute of Technology, Atlanta, GA 30332, USA; \\ email: hani@math.gatech.edu \\ ${ }^{2}$ Université Paris-Nord, France; \\ email: pausader@math.univ-paris13.fr \\ ${ }^{3}$ Université Cergy-Pontoise, France; \\ email: nikolay.tzvetkov@u-cergy.fr \\ ${ }^{4}$ Universita di Pisa, Italy; \\ email: viscigli@dm.unipi.it
}

Received 8 October 2014; accepted 7 July 2015

\begin{abstract}
We consider the cubic nonlinear Schrödinger equation posed on the spatial domain $\mathbb{R} \times \mathbb{T}^{d}$. We prove modified scattering and construct modified wave operators for small initial and final data respectively $(1 \leqslant d \leqslant 4)$. The key novelty comes from the fact that the modified asymptotic dynamics are dictated by the resonant system of this equation, which sustains interesting dynamics when $d \geqslant 2$. As a consequence, we obtain global strong solutions (for $d \geqslant 2$ ) with infinitely growing high Sobolev norms $H^{s}$.
\end{abstract}

2010 Mathematics Subject Classification: 35Q55

\section{Introduction}

The purpose of this work is to study the asymptotic behavior of the cubic defocusing nonlinear Schrödinger (NLS) equation posed on the waveguide manifolds $\mathbb{R} \times \mathbb{T}^{d}$ :

$$
\left(i \partial_{t}+\Delta\right) U=|U|^{2} U
$$

(C) The Author(s) 2015. This is an Open Access article, distributed under the terms of the Creative Commons Attribution licence (http://creativecommons.org/licenses/by/4.0/), which permits unrestricted re-use, distribution, and reproduction in any medium, provided the original work is properly cited. 
where $U$ is a complex-valued function on the spatial domain $(x, y) \in \mathbb{R} \times \mathbb{T}^{d}$. In particular, we want to understand how this asymptotic behavior is related to a resonant dynamic, in a case when scattering does not occur. Our results can be directly extended to the case of a focusing nonlinearity $\left(-|U|^{2} U\right.$ in the lefthand side of (1.1)), but we will however be concerned with small data, so this distinction on the nonlinearity will not be relevant. On the other hand, the result of Corollary 1.4 providing solutions blowing up at infinite time is more striking in the defocusing case, because in the focusing case one may have blow-up in finite time (via the quite different mechanism of self-focusing).

1.1. Motivation and background. The question of the influence of the geometry on the global behavior of solutions to the nonlinear Schrödinger equation

$$
\left(i \partial_{t}+\Delta\right) u=\lambda|u|^{p-1} u, \quad p>1
$$

dates back at least to [20]. The first natural question is the issue of global existence of solutions, and many works have investigated this problem in different geometric settings [3-5, 13, 18, 22-24, 29, 36, 49, 51, 56-61, 67, 69, $70,75,82,87]$. The conclusion that could be derived from these works is that the geometry of the spatial domain turned out to be of importance in the context of the best possible Strichartz inequalities or the sharp local in time well-posedness results (see for example [5, 21, 22]). However, the analysis in [57-61, 75] seems to indicate that, at least in the defocusing case (in the focusing case, and for large data, it is likely that existence or nonexistence questions to elliptic problems also plays an important role), the only relevant geometric information for the global existence in the energy space is the 'local dimension', that is, the dimension of the tangent plane.

The next natural question concerns the asymptotic behavior. There, the geometry must play a more important role. This is the question in which we are interested in this paper, focusing on the simpler case of noncompact quotients of $\mathbb{R}^{d}$.

When the domain is the Euclidean space, $\mathbb{R}^{d}$, this question is reasonably well understood, at least when the nonlinearity is defocusing and analytic ( $p$ odd integer). In this case, global smooth solutions disperse and in many cases even scatter to a linear state (possibly after modulation by a real phase when $d=1$, $p=3)[27,29,32,34,35,54,67,68,74,79,87,89]$.

In contrast, much less is known for compact domains. The most studied example is that of the torus $\mathbb{T}^{d}$. In this case, many different long-time behaviors can be sustained even on arbitrarily small open sets around zero, ranging from KAM tori $[11,37,72,78]$ to heteroclinic orbits $[30,45]$ and coherent out-ofequilibrium frequency dynamics (interestingly, all these long-time results derive 
from an analysis of resonant interactions that will play a central role in this work as well) [38]. One may also mention $[6,9,19,84]$, where invariant measures for (1.1) are constructed, when the problem is posed on $\mathbb{T}^{d}, d=1,2$, or the $d$-dimensional ball for $d=2,3$ (with radial data). These works establish the existence of a large set of (not necessarily small) recurrent dynamics of (1.1).

In light of the above sharp contrast in behavior between $\mathbb{R}^{d}$ and its compact quotient $\mathbb{T}^{d}$, considerable interest has emerged in the past few years to study questions of long-time behavior on 'in-between' manifolds, like the ones presented by the noncompact quotients of Euclidean space $[\mathbf{5 1}, \mathbf{5 8}, \mathbf{5 9}, \mathbf{8 2}, \mathbf{8 3}$, 85].

In the generality of noncompact Riemannian $d$-manifolds $M$, it seems plausible that a key role is played by the parameter $\alpha$ for which solutions to the linear NLS equation ((1.2) with $\lambda=0)$ with smooth compactly supported initial data decay like $t^{-\alpha / 2}$. In light of the Euclidean theory on $\mathbb{R}^{\alpha}$, one can draw the following hypothetical heuristics: (H1) when $p>1+4 / \alpha$, global solutions (sufficiently small in the focusing case) scatter, and no further information is needed about the geometry 'at infinity'; (H2) if $p=1+4 / \alpha$, global solutions scatter, but the geometry 'at infinity' plays an important role in the analysis of certain sets of solutions (for example in the profile decomposition); (H3) if $p \leqslant 1+2 / \alpha$, no nontrivial solution can scatter; and (H4) if $p=1+2 / \alpha$, global solutions exhibit some 'modified scattering' characterized by a correction to scattering on a larger time scale. We are interested in this latter regime to which (1.1) belongs.

In support of the heuristic (H1), we cite the results in $[3,61,65,85,86]$. The second heuristic $(\mathrm{H} 2)$ was put to test in [51], where the authors study the quintic NLS equation on $\mathbb{R} \times \mathbb{T}^{d}$. There, a strong relation is drawn between the large-data scattering theory for the quintic NLS equation and the system obtained from its resonant periodic frequency interactions. The relevance of the result in [51] to our work here lies in the following two important messages. The first is that the asymptotic behavior of (1.2) on $\mathbb{R}^{n} \times \mathbb{T}^{d}$ can be understood through (i) the asymptotic dynamics of the same equation on Euclidean spaces, and (ii) the asymptotic dynamics of a related resonant system corresponding to the resonant interactions between its periodic frequency modes. The second message from [51] is the insight that the resonant interactions in (1.1) will play a vivid and decisive role in dictating the anticipated nonscattering asymptotic dynamics of (1.1). Indeed, as [51, 85] show that quintic interactions lead to scattering behavior for small data, and since nonresonant interactions in (1.1) can be transformed, at least formally, into quintic interactions via a normal form transformation, it is up to the resonant interactions alone to drive the system away from scattering. This is the content of our main result. 
The fact that resonant interactions describe the long-time dynamics of the system has very important consequences. Most notably, it allows one to construct solutions of (1.1) that exhibit infinite cascades of energy from low frequencies toward high frequencies based on earlier constructions for the resonant system (see Corollary 1.4). Such cascades are expected on $\mathbb{T}^{d}$ (that is, without the noncompact component), but a similar strategy fails there, precisely because no such description of the asymptotic behavior in terms of resonant interactions can be made.

The other interesting feature of the asymptotic dynamics of (1.1) as opposed to previous modified scattering results is that the modification dictated by its resonant system is not simply a phase correction term when $d \geqslant 2$, but rather a much more vigorous departure from linear dynamics. As we argue below, this will pose a new set of difficulties in comparison to previous modified scattering results in the literature, but, on the plus side, will lead us to several interesting and new types of asymptotic dynamics.

1.2. Statement of the results. Consistent with the heuristics above, we show that the asymptotic dynamic of small solutions to (1.1) is related to that of solutions of the resonant system

$$
\begin{aligned}
i \partial_{\tau} G(\tau) & =\mathcal{R}[G(\tau), G(\tau), G(\tau)], \\
\mathcal{F}_{\mathbb{R} \times \mathbb{T}^{d}} \mathcal{R}[G, G, G](\xi, p) & =\sum_{\substack{p_{1}+p_{3}=p+p_{2} \\
\left|p_{1}\right|^{2}+\left|p_{3}\right|^{2}=|p|^{2}+\left|p_{2}\right|^{2}}} \widehat{G}\left(\xi, p_{1}\right) \overline{\widehat{G}\left(\xi, p_{2}\right)} \widehat{G}\left(\xi, p_{3}\right) .
\end{aligned}
$$

Here, $\widehat{G}(\xi, p)=\mathcal{F}_{\mathbb{R} \times \mathbb{T}^{d}} G(\xi, p)$ is the Fourier transform of $G$ at $(\xi, p) \in \mathbb{R} \times \mathbb{Z}^{d}$. Noting that the dependence on $\xi$ is merely parametric, the above system is none other than the resonant system for the cubic NLS equation on $\mathbb{T}^{d}$. Equation (1.3) is globally well posed thanks to Lemma 4.1 below.

More precisely, our main results are as follows. Below, $N \geqslant 30$ is an arbitrary integer, and $S$ and $S^{+}$denote Banach spaces whose norms are defined in (2.8) later. They contain all the Schwartz functions.

TheOrem 1.1. Let $1 \leqslant d \leqslant 4$. There exists $\varepsilon=\varepsilon(N, d)>0$ such that, if $U_{0} \in S^{+}$satisfies

$$
\left\|U_{0}\right\|_{S^{+}} \leqslant \varepsilon
$$

and if $U(t)$ solves $(1.1)$ with initial data $U_{0}$, then $U \in C\left((0,+\infty): H^{N}\right)$ exists globally and exhibits modified scattering to its resonant dynamics (1.3) in the following sense: there exists $G_{0} \in S$ such that, if $G(t)$ is the solution of (1.3) with initial data $G(0)=G_{0}$, then

$$
\left\|U(t)-e^{i t \Delta_{\mathbb{R} \times \mathbb{T}^{d}}} G(\pi \ln t)\right\|_{H^{N}\left(\mathbb{R} \times \mathbb{T}^{d}\right)} \rightarrow 0 \quad \text { as } t \rightarrow+\infty .
$$


Moreover,

$$
\|U(t)\|_{L_{x}^{\infty} H_{y}^{1}} \lesssim(1+|t|)^{-1 / 2} .
$$

A similar statement holds as $t \rightarrow-\infty$, and a more precise one is contained in Theorem 6.1. It is worth pointing out that, for $d=4$, even the global existence claim in the above theorem is new, due to the energy-supercritical nature of (1.1) in this dimension. However, the main novelty is the modified scattering statement to a nonintegrable asymptotic dynamics, given by (1.3).

In addition, we construct modified wave operators in the following sense.

THEOREM 1.2. Let $1 \leqslant d \leqslant 4$. There exists $\varepsilon=\varepsilon(N, d)>0$ such that, if $G_{0} \in S^{+}$satisfies

$$
\left\|G_{0}\right\|_{S^{+}} \leqslant \varepsilon
$$

and $G(t)$ solves (1.3) with initial data $G_{0}$, then there exists $U \in C\left((0, \infty): H^{N}\right)$, a solution of (1.1) such that

$$
\left\|U(t)-e^{i t \Delta_{\mathbb{R} \times \mathbb{T}^{d}}} G(\pi \ln t)\right\|_{H^{N}\left(\mathbb{R} \times \mathbb{T}^{d}\right)} \rightarrow 0 \quad \text { as } t \rightarrow+\infty .
$$

REMARK 1.3. It is worth mentioning that a slight modification of the proof of Theorems 1.1 and 1.2 shows that similar statements hold if $\mathbb{T}^{d}$ is replaced by the sphere $S^{d}, d=2,3$ (with a suitably modified resonant system). Indeed, the largest part of the analysis is exploiting the one-dimensional dispersion. In the case of $S^{d}, d=2,3$, the spectrum of the Laplace-Beltrami operator satisfies the nonresonant condition needed for the normal form analysis, and the $H^{1}$ wellposedness analysis on the sphere of $[22,24]$ provides the needed substitute of Lemma 7.1. We note however that a good understanding of the corresponding resonant system is still lacking. Modifications of the proof also hold in the case of a partial harmonic confinement, and there the resonant system is better understood (see [52]). On the other hand, the extension of our analysis to an irrational torus is less clear, because of the appearance of small denominators in the normal form analysis.

As a consequence of Theorem 1.2, as detailed in Remark 4.4, all the behaviors that can be isolated for solutions of the resonant system (1.3) have counterparts in the asymptotic behavior of solutions of (1.1). Most notably, given the existence of unbounded Sobolev orbits for (1.3) as proved in [50] for $d \geqslant 2$ (see Theorem 4.8 for an explicit construction with quantitative lower bounds on the growth), we have the following.

COROLlaRy 1.4 (Existence of infinite cascade solutions). Let $d \geqslant 2$ and $s \in \mathbb{N}$, $s \geqslant 30$. Then for every $\varepsilon>0$ there exists a global solution $U(t)$ of (1.1) such 
that

$$
\|U(0)\|_{H^{s}\left(\mathbb{R} \times \mathbb{T}^{d}\right)} \leqslant \varepsilon, \quad \limsup _{t \rightarrow+\infty}\|U(t)\|_{H^{s}\left(\mathbb{R} \times \mathbb{T}^{d}\right)}=+\infty
$$

More precisely, there exist a sequence $t_{k} \rightarrow+\infty$ and a constant $C$ such that

$$
\left\|U\left(t_{k}\right)\right\|_{H^{s}\left(\mathbb{R} \times \mathbb{T}^{d}\right)} \gtrsim\left(\log \log t_{k}\right)^{C}
$$

REMARK 1.5. These infinite cascades do not occur when $d=1$ on $\mathbb{R} \times \mathbb{T}$ (nor when $d=0$ on $\mathbb{R}$ ), at least not for small smooth localized solutions. In fact (see (4.8)), the asymptotic dynamic of small solutions to (1.1) is fairly similar on $\mathbb{R}$ and on $\mathbb{R} \times \mathbb{T}$, in sharp contrast with the case when $d \geqslant 2$.

REMARK 1.6. The growth in the above corollary only happens over a sequence of times $t_{k} \rightarrow \infty$. It would be interesting to investigate whether this growth can be made uniform (if this were possible at all).

Corollary 1.4 gives a partial solution to a problem posed by Bourgain [16, page 43-44] concerning the possible long-time growth of the $H^{s}, s>1$ norms for the solutions of the cubic nonlinear Schrödinger equation. This growth of high Sobolev norms is regarded as a proof of the (direct) energy cascade phenomenon in which the energy of the system (here, the kinetic energy) moves from low frequencies (large scales) toward arbitrarily high frequencies (small scales). Heuristically, the solution in Corollary 1.4 can be viewed as initially oscillating at scales that are $O(1)$, but at later times it exhibits oscillations at arbitrarily smaller length scales. This energy cascade is a main aspect of the outof-equilibrium dynamics predicted for (1.1) by the vast literature of physics and numerics falling under the theory of weak (wave) turbulence (see [73, 90]).

The corresponding result on $\mathbb{T}^{d}$ does not directly follow from Corollary 1.4 (nor does it imply it). This is somehow surprising, because one would naturally expect that adding a dispersive direction to $\mathbb{T}^{d}$ would drive the system closer to nonlinear asymptotic stability, and further from out-of-equilibrium dynamics (this is indeed the case if we study the equation on $\mathbb{R}^{n} \times \mathbb{T}^{d}$ for $n \geqslant 2$, as was shown by the scattering result in [85]). Our construction draws heavily on $[30,45,50]$, where unbounded Sobolev orbits are constructed for the resonant system and applied to get finite-time amplifications of the Sobolev norms on $\mathbb{T}^{2}$. However, in the case of the torus, nonresonant interactions do not disappear and feed back into the dynamics after a long but finite time. This is precisely where the more dispersive setting of $\mathbb{R} \times \mathbb{T}^{d}$ makes a difference: in this case, nonresonant terms are transformed into quintic terms which scatter, and hence, at least heuristically, do not modify the long-term dynamics. 
Previous results in the spirit of Corollary 1.4 may be found in $[14,15]$ for linear Schrödinger equations with potential, [30, 45, 71] for finite-time amplifications of the initial $H^{s}$ norm, $[7,10,50]$ for NLS with suitably chosen nonlocal nonlinearities, and [39-41, 76, 77, 88] for the zero-dispersion Szegö and half-wave equations. Concerning the opposite question of obtaining upper bounds on the rate of possible growth of the Sobolev norms of solutions of NLS equations, we refer to $[8,17,31,80,81]$.

One can also use Theorem 1.2 to construct other interesting nonscattering dynamics for Equation (1.1), as is illustrated in the following result.

COROLlaRY 1.7 (Forward compact solutions). Let $d \geqslant 2$. For functions $U(t)$ on $\mathbb{R} \times \mathbb{T}^{d}$ defined for all $t \geqslant 0$, we define the 'limit profile set' as

$$
\omega(U)=\limsup _{t \rightarrow+\infty}\left\{e^{-i t \Delta_{\mathbb{R} \times \mathbb{T}^{d}}} U(t)\right\}=\bigcap_{\tau \in(0, \infty)} \overline{\left\{e^{\left.-i t \Delta_{\mathbb{R} \times \mathbb{T}^{d}} U(t): t \geqslant \tau\right\}} .\right.}
$$

Then we have the following.

(1) (No nontrivial scattering) Assume that $U$ solves (1.1) and that $\omega(U)$ is a point. If $U(0)$ is sufficiently small, then $U \equiv 0$.

(2) (Scattering up to phase correction) There exist a nontrivial solution $U$ of (1.1) and a real function $b: \mathbb{R} \rightarrow \mathbb{R}$ such that $\omega\left(U(t) e^{i b(t)}\right)$ is a point.

(3) ((Quasi)periodic frequency dynamics) There exists a global solution $U(t)$ such that $\omega(U)$ is compact but $\operatorname{dim} \operatorname{Span}(\omega(U)) \geqslant 2$.

The proof of part (3) in the above corollary is interesting in its own right. In fact, we construct global solutions to (1.1) that asymptotically bounce their energy (and mass) between two disjoint sites in frequency space periodically in time. These correspond to periodic-in-time solutions of (1.3) that exhibit the following 'beating effect' (in the nomenclature of [44]): there exist two disjoint subsets $\mathbb{R} \times \Lambda_{1}$ and $\mathbb{R} \times \Lambda_{2}$ in $\mathbb{R} \times \mathbb{Z}^{2}$, so that for any $\varepsilon \in(0,1)$ there exists a solution $G(t)$ of (1.3) that is supported in frequency space on $\mathbb{R} \times \Lambda_{1} \cup \mathbb{R} \times$ $\Lambda_{2}$ in such a way that the fraction of the mass carried by each of the two sets alternates between $\varepsilon$ and $1-\varepsilon$ periodically in time. We refer to Section 4.2 for more constructions including asymptotically quasiperiodic dynamics.

1.2.1. Comments. It would be interesting to understand what is the optimal topology to obtain our results. It is probably a lot larger than the one we use. Progress in this direction would have the following impact. 
- The results are restricted to small data. In the absence of a 'correct' topology, the exact meaning of "large data" is not well established.

- We cannot let any $H^{s}$ norm, $s>1$, grow in Corollary 1.4, partly because we want to cover all the cases $0 \leqslant d \leqslant 4$ in a uniform manner, using simple exponents. More careful analysis might address this point (for instance, either lowering the regularity requirement in Theorem 1.2 or a more quantified version of the construction in [30] would resolve this). We decided not to pursue this point here because Corollary 1.4 already captures the energy cascade phenomenon.

- It is possible that a more adapted topology allows one to define the scattering operator in a good Banach space.

Finally, we also mention the situation in [2], where the partial periodicity is replaced by adding a (partially) confining potential.

\subsection{Overview of proof.}

1.3.1. Modified scattering. In order to describe the asymptotic behavior of a nonlinear dispersive equation like (1.1), it may be relevant to study the limiting behavior of the profile $F(t)=e^{-i t \Delta} U(t)$ obtained by conjugating out the linear flow. If $F(t)$ converges to a fixed function $G_{\infty}$, then the solution scatters. If not, the next best thing is to find the simplest possible dynamical system that describes the asymptotic dynamics of $F(t)$. To find this system, one has to work on proving global a priori energy and decay estimates that allow one to decompose the nonlinearity in the $F$ equation in the following way:

$$
i \partial_{t} F=\mathcal{N}(F)=\mathcal{N}_{e f f}(F)+\mathcal{E}(F),
$$

where $\mathcal{E}(F)$ is integrable in time. When this is possible, one can hope to prove that the asymptotic dynamics converge to that of the effective system

$$
i \partial_{t} G=\mathcal{N}_{\text {eff }}(G) .
$$

Proving the global a priori energy and decay estimates can be a daunting task, depending on the problem at hand. On the other hand, the process of proving the convergence to the dynamics of (1.6) depends very much on how simple or complicated $\mathcal{N}_{\text {eff }}(G)$ is.

Previous modified scattering results that we are aware of only concerned equations (or systems) posed on $\mathbb{R}^{d}$, quasilinear or semilinear $[1,25,32,33,53$ 55, 63, 64, 66, 74, 89], and had an integrable asymptotic system for (1.6). 
This often allowed for a simple phase conjugation (in physical or Fourier space) to give the modification. In contrast, our limiting system is given by (1.3), which is not only a nonintegrable system, but also allows for the growth of norms of its solutions, as we saw in Corollary 1.4. This requires a robust approach to modified scattering that tolerates the growth of the limiting system (1.6) as long as the decay of $\mathcal{E}(F)$ in (1.5) is sufficiently fast to trump the divergence effects of the effective part $\mathcal{N}_{\text {eff }}(F)$.

1.3.2. Isolating the resonant system: heuristics. To isolate the effective interactions $\left(\mathcal{N}_{\text {eff }}\right.$ above), we can argue formally by looking at (1.1) in Fourier space:

$i \partial_{t} \widehat{F}_{p}(\xi, t)=\sum_{q-r+s=p} e^{i \omega t} \int_{\mathbb{R}^{2}} e^{2 i \eta \kappa t} \widehat{F}_{q}(\xi-\eta, t) \widehat{\widehat{F}}_{r}(\xi-\eta-\kappa, t) \widehat{F}_{s}(\xi-\kappa, t) d \kappa d \eta$,

where $\omega=|p|^{2}-|q|^{2}+|r|^{2}-|s|^{2}$, and where $\widehat{F}_{p}(\xi)$ denotes the Fourier transform of $F$ at $(\xi, p) \in \mathbb{R} \times \mathbb{Z}^{d}$. Roughly speaking, a stationary phase argument in the $(\eta, \kappa)$ integral implies (see [66] for a previous use of this remark) that for very large times the equation for $\widehat{F}_{p}(\xi, t)$ can be written as

$$
i \partial_{t} \widehat{F}_{p}(\xi, t)=\frac{\pi}{t} \sum_{q-r+s=p} e^{i \omega t} \widehat{F}_{q}(\xi, t) \widehat{F}_{r}(\xi, t) \widehat{F}_{s}(\xi, t)+\text { l.o.t. }
$$

This is essentially an ordinary differential equation (ODE) system for each $\xi \in \mathbb{R}$. As is well known, resonant interactions corresponding to $(p, q, r, s)$ for which $\omega=0$ play a particularly important role in the dynamics of such an ODE, especially given the decay of $\partial_{t} \widehat{F}_{p}$. This suggests that the expression above can be simplified to

$$
i \partial_{t} \widehat{F}_{p}(\xi, t)=\frac{\pi}{t} \sum_{\substack{q-r+s=p \\|q|^{2}-|r|^{2}+|s|^{2}=|p|^{2}}} \widehat{F}_{q}(\xi, t) \widehat{\widehat{F}}_{r}(\xi, t) \widehat{F}_{s}(\xi, t)+\text { l.o.t. }
$$

As a result, one should expect the asymptotic dynamics of $F$ to be dictated by the ODE system given by the first term on the right-hand side above. The latter system can be seen to be autonomous when written in terms of the slow time scale $\tau=\pi \ln t$ in which it has the form (1.3). Note that this system was previously studied and shown to have interesting dynamics [26, 30, 38, 50].

The upshot of the above formal calculation is that one should expect a solution $F(t)$ to $(1.7)$ to asymptote to some $G(\pi \ln t)$ where $G(\tau)$ solves (1.3). This is the content of Theorem 1.1. 
1.3.3. Norms and the control of the solution. As mentioned above, establishing a priori energy and decay estimates is a precursor to isolating the leading-order dynamics. In the scalar case $d=0[54,66]$, the needed energy estimates follow easily once we guarantee the $t^{-1 / 2}$ decay for the $L^{\infty}$ norm. Indeed, schematically speaking, if $E(t)$ is an appropriate energy of the system that controls its strong norms, then one has the relation

$$
\partial_{t} E(t) \lesssim\|u(t)\|_{L^{\infty}}^{2} E(t) \lesssim t^{-1} E(t)
$$

which barely allows one to close any polynomial-growth bootstrap for $E(t)$. The $L^{\infty}$ decay can be bootstrapped by relying on the boundedness of the Fourier transform, which follows from the equation satisfied by $\widehat{F}(\xi)$. An almost identical energy method argument works in the case when $d=1$, but it reaches its limit there. Indeed, for $d \geqslant 2$, we do not have access to the sharp linear decay $t^{-1 / 2}$ which was crucial to closing the energy bootstrap above. To overcome this difficulty, we need additional estimates coming from the low-regularity theory. We use a hierarchy of three norms.

- The Z-norm is bounded and essentially corresponds to the strongest information that remains a priori bounded uniformly in time.

- The $S$-norm controls the number of periodic derivatives we want to consider. It grows slowly with time, but the difference between the solutions and the asymptotic dynamics decays in this norm.

- The $S^{+}$-norm is slightly stronger than the $S$-norm. It is allowed to grow slowly, but still yields better control on objects in the $S$-norm. In particular, it controls the same number of derivatives in the periodic directions as the $S$-norm.

While the choice of the $Z$-norm is dictated by the resonant system, there is considerable flexibility in the choices of the two other norms. Another possible choice might be a variation (but for the moment, it seems difficult in the proof of the modified wave operator to work with an intermediate norm controlling no weight in $x$ ) of $Z, Z \cap H^{N}, S$. One of the main problems complicating the situation here is the need for a bounded linearized operator around a solution $G$ of the asymptotic system, which is not trivial in view of the missing $t^{-1 / 2}$ decay of $\|U(t)\|_{L^{\infty}}$.

The significance of the $Z$-norm stems from the following two key facts: (1) it is conserved for the resonant system (ultimately, this leads to the key nonperturbative ingredient; see (4.4) and (6.5)), and (2) it is a controlling norm for the existence and growth of its solution (in particular, this forces the restriction $d \leqslant 4$ in Theorems 1.1 and 1.2) in view of Lemma 4.3. This, combined 
with Lemma 7.3, provides the extent to which we can get decay for solutions of (1.1). Interestingly, all this global analysis of the resonant system (1.3) relies heavily on using local-in-time Strichartz estimates on the torus in order to get global-in-time bounds for the $Z$-norm of the nonlinearity (see Lemma 7.1). At this place our viewpoint is quite different from a naïve one-dimensional vectorvalued analysis (as is the case in [85]).

We also note that, although our approach is close in spirit to recent developments in global existence for quasilinear equations $[42,43,48,62-$ 64], some of the key estimates really pertain to the low-regularity theory (see Lemma 7.1 and Lemma 7.2 (this is somewhat parallel to the energy method in the quasilinear results)).

Organization of the paper. Section 2 introduces the notation used in this paper. Section 3 provides a decomposition of the nonlinearity as in (1.5). Section 4 introduces the resonant system (1.3) and gives some properties of its solutions. Section 5 shows existence of the modified wave operators and proves Theorem 1.2. Section 6 shows the modified scattering statement and proves Theorem 1.1. Finally, Section 7 collects various additional estimates needed in the proofs.

\section{Notation}

2.1. Standard notation. In this paper, $\mathbb{T}:=\mathbb{R} /(2 \pi \mathbb{Z})$. We will often consider functions $f: \mathbb{R} \rightarrow \mathbb{C}$ and functions $F: \mathbb{R} \times \mathbb{T}^{d} \rightarrow \mathbb{C}$. To distinguish between them, we use the convention that lower-case letters denote functions defined on $\mathbb{R}$, upper-case letters denote functions defined on $\mathbb{R} \times \mathbb{T}^{d}$, and calligraphic letters denote operators, except for the Littlewood-Paley operators and dyadic numbers, which are capitalized most of the time.

We define the Fourier transform on $\mathbb{R}$ by

$$
\widehat{g}(\xi):=\frac{1}{2 \pi} \int_{\mathbb{R}} e^{-i x \xi} g(x) d x .
$$

Similarly, if $F(x, y)$ depends on $(x, y) \in \mathbb{R} \times \mathbb{T}^{d}, \widehat{F}(\xi, y)$ denotes the partial Fourier transform in $x$. We also consider the Fourier transform of $h: \mathbb{T}^{d} \rightarrow \mathbb{C}$,

$$
h_{p}:=\frac{1}{(2 \pi)^{d}} \int_{\mathbb{T}^{d}} h(y) e^{-i\langle p, y\rangle} d y, \quad p \in \mathbb{Z}^{d},
$$

and this extends to $F(x, y)$. Finally, we also have the full (spatial) Fourier transform

$$
(\mathcal{F} F)(\xi, p)=\frac{1}{(2 \pi)^{d}} \int_{\mathbb{T}^{d}} \widehat{F}(\xi, y) e^{-i\langle p, y\rangle} d y=\widehat{F}_{p}(\xi) .
$$


We will often use Littlewood-Paley projections. For the full frequency space, these are defined as follows:

$$
\left(\mathcal{F} P_{\leqslant N} F\right)(\xi, p)=\varphi\left(\frac{\xi}{N}\right) \varphi\left(\frac{p_{1}}{N}\right) \ldots \varphi\left(\frac{p_{d}}{N}\right)(\mathcal{F} F)(\xi, p),
$$

where $\varphi \in C_{c}^{\infty}(\mathbb{R}), \varphi(x)=1$ when $|x| \leqslant 1$ and $\varphi(x)=0$ when $|x| \geqslant 2$. Next, we define

$$
P_{N}=P_{\leqslant N}-P_{\leqslant N / 2}, \quad P_{\geqslant N}=1-P_{\leqslant N / 2} .
$$

Many times we will concentrate on the frequency in $x$ only, and we therefore define

$$
\left(\mathcal{F} Q_{\leqslant N} F\right)(\xi, p)=\varphi\left(\frac{\xi}{N}\right)(\mathcal{F} F)(\xi, p),
$$

and define $Q_{N}$ similarly to $P_{N}$. By a slight abuse of notation, we will consider $Q_{N}$ indifferently as an operator on functions defined on $\mathbb{R} \times \mathbb{T}^{d}$ and on $\mathbb{R}$. We shall use the following commutator estimate:

$$
\left\|\left[Q_{N}, x\right]\right\|_{L_{x}^{2} \rightarrow L_{x}^{2}} \lesssim N^{-1} .
$$

Below, we will need a few parameters. For $T \gtrsim 1$ a positive number, we let $q_{T}: \mathbb{R} \rightarrow \mathbb{R}$ be an arbitrary function satisfying

$$
\begin{gathered}
0 \leqslant q_{T}(s) \leqslant 1, \quad q_{T}(s)=0 \text { if }|s| \leqslant T / 4 \text { or }|s| \geqslant T, \quad \text { and } \\
\int_{\mathbb{R}}\left|q_{T}^{\prime}(s)\right| d s \leqslant 10 .
\end{gathered}
$$

Particular examples are the characteristic functions $q_{T}(s)=1_{[T / 2, T]}(s)$, with the natural interpretation of the integral on $\mathbb{R}$ of $\left|q_{T}^{\prime}\right|$.

We will use the following sets corresponding to momentum and resonance level sets:

$$
\begin{aligned}
\mathcal{M} & :=\left\{(p, q, r, s) \in \mathbb{Z}^{4 d}: p-q+r-s=0\right\}, \\
\Gamma_{\omega} & :=\left\{(p, q, r, s) \in \mathcal{M}:|p|^{2}-|q|^{2}+|r|^{2}-|s|^{2}=\omega\right\} .
\end{aligned}
$$

In particular, note that $(p, q, r, s) \in \Gamma_{0}$ if and only if $\{p, q, r, s\}$ are the vertices of a rectangle.

2.2. Duhamel formula. We will prove all our statements for $t \geqslant 0$. By timereversal symmetry, one obtains the analogous claims for $t \leqslant 0$. In studying solutions to (1.1), it will be convenient to factor out the linear flow and write a solution $U$ of (1.1) as

$$
U(x, y, t)=\sum_{p \in \mathbb{Z}^{d}} e^{i\langle p, y\rangle} e^{-i t|p|^{2}}\left(e^{i t \partial_{x x}} F_{p}(t)\right)(x)=e^{i t \Delta_{\mathbb{R} \times \mathbb{T}^{d}}}(F(t)) .
$$


We then see that $U$ solves (1.1) if and only if $F$ solves

$$
i \partial_{t} F(t)=e^{-i t \Delta_{\mathbb{R} \times \mathbb{T}^{d}}}\left(e^{i t \Delta_{\mathbb{R} \times \mathbb{T}^{d}}} F(t) \cdot e^{-i t \Delta_{\mathbb{R} \times \mathbb{T}^{d}}} \overline{F(t)} \cdot e^{i t \Delta_{\mathbb{R} \times \mathbb{T}^{d}}} F(t)\right) .
$$

Note in particular that $F$ evolves purely nonlinearly and that $\partial_{t} F$ is 'smaller' than $F$ in many ways, as partly captured in our choice of norms. We will denote the nonlinearity in (2.3) by $\mathcal{N}^{t}[F(t), F(t), F(t)]$, where the trilinear form $\mathcal{N}^{t}$ is defined by

$$
\mathcal{N}^{t}[F, G, H]:=e^{-i t \Delta_{\mathbb{R} \times \mathbb{T}^{d}}}\left(e^{i t \Delta_{\mathbb{R} \times \mathbb{T}^{d}}} F \cdot e^{-i t \Delta_{\mathbb{R} \times \mathbb{T}^{d}}} \bar{G} \cdot e^{i t \Delta_{\mathbb{R} \times \mathbb{T}^{d}}} H\right) .
$$

Now, we can compute the Fourier transform of the last expression, which leads to the identity

$$
\mathcal{F} \mathcal{N}^{t}[F, G, H](\xi, p)=\sum_{(p, q, r, s) \in \mathcal{M}} e^{i t\left[|p|^{2}-|q|^{2}+|r|^{2}-|s|^{2}\right]} \mathcal{I}^{t}\left[\widehat{F_{q}, G_{r}}, H_{s}\right](\xi),
$$

where

$$
\mathcal{I}^{t}[f, g, h]:=\mathcal{U}(-t)(\mathcal{U}(t) f \overline{\mathcal{U}(t) g} \mathcal{U}(t) h), \quad \mathcal{U}(t)=\exp \left(i t \partial_{x}^{2}\right) .
$$

One verifies that

$$
\left.\mathcal{I}^{t} \widehat{[f, g}, h\right](\xi)=\int_{\mathbb{R}^{2}} e^{i t 2 \eta \kappa} \widehat{f}(\xi-\eta) \widehat{g}(\xi-\eta-\kappa) \widehat{h}(\xi-\kappa) d \kappa d \eta .
$$

Thus one may also write

$$
\begin{aligned}
\mathcal{F} \mathcal{N}^{t}[F, G, H](\xi, p)= & \sum_{(p, q, r, s) \in \mathcal{M}} e^{i t\left[|p|^{2}-|q|^{2}+|r|^{2}-|s|^{2}\right]} \\
& \times \int_{\mathbb{R}^{2}} e^{i t 2 \eta \kappa} \widehat{F}_{q}(\xi-\eta) \widehat{\widehat{G}}_{r}(\xi-\eta-\kappa) \widehat{H}_{s}(\xi-\kappa) d \kappa d \eta .
\end{aligned}
$$

According to our previous discussion, we now define the resonant part of the nonlinearity $\left((\pi / t) \mathcal{R}\right.$ corresponds to $\mathcal{N}_{\text {eff }}$ in (1.5)) as

$$
\mathcal{F} \mathcal{R}[F, G, H](\xi, p):=\sum_{(p, q, r, s) \in \Gamma_{0}} \widehat{F}_{q}(\xi){\widehat{G_{r}}}_{r}(\xi) \widehat{H}_{s}(\xi) .
$$

We have a remarkable Leibniz rule for $\mathcal{I}^{t}[f, g, h]$, namely

$$
Z \mathcal{I}^{t}[f, g, h]=\mathcal{I}^{t}[Z f, g, h]+\mathcal{I}^{t}[f, Z g, h]+\mathcal{I}^{t}[f, g, Z h], \quad Z \in\left\{i x, \partial_{x}\right\} .
$$

A similar property holds for the whole nonlinearity $\mathcal{N}^{t}[F, G, H]$, where $Z$ can also be a derivative in the transverse direction, $Z=\partial_{y_{j}}$. Property (2.7) will be of importance in order to ensure the hypothesis of the transfer principle displayed by Lemma 7.4 . 
2.3. Norms. Our norms will depend on two parameters which we fix throughout the paper. We set $\delta<10^{-3}$ and $N \geqslant 30$ (the exact value of $N$ can be significantly lowered for example by allowing more weights in the $S$-norm in (2.8)). We will often consider sequences, and we define the following norm on these:

$$
\left\|\left\{a_{p}\right\}\right\|_{h_{p}^{s}}^{2}:=\sum_{p \in \mathbb{Z}^{d}}\left[1+|p|^{2}\right]^{s}\left|a_{p}\right|^{2} .
$$

For functions, we will often omit the domain of integration from the description of the norms. However, we will indicate it by a subscript $x$ (for $\mathbb{R}$ ), $x, y$ (for $\mathbb{R} \times \mathbb{T}^{d}$ ) or $p\left(\right.$ for $\mathbb{Z}^{d}$ ). We will use mainly three different norms: a weak norm,

$$
\|F\|_{Z}^{2}:=\sup _{\xi \in \mathbb{R}}\left[1+|\xi|^{2}\right]^{2} \sum_{p \in \mathbb{Z}^{d}}[1+|p|]^{2}\left|\widehat{F}_{p}(\xi)\right|^{2}=\sup _{\xi \in \mathbb{R}}\left[1+|\xi|^{2}\right]^{2}\left\|\widehat{F}_{p}(\xi)\right\|_{h_{p}^{1}}^{2},
$$

and two strong norms,

$$
\|F\|_{S}:=\|F\|_{H_{x, y}^{N}}+\|x F\|_{L_{x, y}^{2}}, \quad\|F\|_{S^{+}}:=\|F\|_{S}+\left\|\left(1-\partial_{x x}\right)^{4} F\right\|_{S}+\|x F\|_{S} .
$$

We have the following hierarchy:

$$
\|F\|_{H_{x, y}^{1}} \lesssim\|F\|_{Z} \lesssim\|F\|_{S} \lesssim\|F\|_{S^{+}}
$$

To verify the middle inequality, using (2.1) and the elementary inequality,

$$
\|f\|_{L_{x}^{1}(\mathbb{R})} \lesssim\|f\|_{L_{x}^{2}(\mathbb{R})}^{1 / 2}\|x f\|_{L_{x}^{2}(\mathbb{R})}^{1 / 2},
$$

one might observe that

$$
\begin{aligned}
{\left[1+|\xi|^{2}\right]|\widehat{F}(\xi, p)| } & \lesssim \sum_{N} N^{2}\left|\widehat{Q_{N} F}(\xi, p)\right| \lesssim \sum_{N} N^{2}\left\|Q_{N} F_{p}\right\|_{L_{x}^{2}}^{1 / 2}\left\|x Q_{N} F_{p}\right\|_{L_{x}^{2}}^{1 / 2} \\
& \lesssim \sum_{N} N^{-1 / 2}\left\|\left(1-\partial_{x x}\right)^{5 / 2} F_{p}\right\|_{L_{x}^{2}}^{1 / 2}\left\|\langle x\rangle F_{p}\right\|_{L_{x}^{2}}^{1 / 2} \\
& \lesssim\left\|F_{p}\right\|_{H_{x}^{5}}^{1 / 2}\left\|\langle x\rangle F_{p}\right\|_{L_{x}^{2}}^{1 / 2} .
\end{aligned}
$$

Squaring and multiplying by $\langle p\rangle^{2}$, we find that (using interpolation too)

$$
\|F\|_{Z} \lesssim\|F\|_{L_{x, y}^{2}}^{1 / 4}\|F\|_{S}^{3 / 4}
$$

We also note that the operators $Q_{\leqslant N}$ and $P_{\leqslant N}$ and the multiplication by $\varphi(\cdot / N)$ are bounded in $Z, S, S^{+}$, uniformly in $N$. 
The space-time norms we will use are

$$
\begin{aligned}
\|F\|_{X_{T}} & :=\sup _{0 \leqslant t \leqslant T}\left\{\|F(t)\|_{Z}+(1+|t|)^{-\delta}\|F(t)\|_{S}+(1+|t|)^{1-3 \delta}\left\|\partial_{t} F(t)\right\|_{S}\right\}, \\
\|F\|_{X_{T}^{+}} & :=\|F\|_{X_{T}}+\sup _{0 \leqslant t \leqslant T}\left\{(1+|t|)^{-5 \delta}\|F(t)\|_{S^{+}}+(1+|t|)^{1-7 \delta}\left\|\partial_{t} F(t)\right\|_{S^{+}}\right\} .
\end{aligned}
$$

In most of the cases, in order to sum up the one-dimensional estimates, we make use of the following elementary bound:

$$
\sum_{(q, r, s):(p, q, r, s) \in \mathcal{M}} c_{q}^{1} c_{r}^{2} c_{s}^{3}\left\|_{l_{p}^{2}} \lesssim \min _{\sigma \in \mathfrak{S}_{3}}\right\| c^{\sigma(1)}\left\|_{l_{p}^{2}}\right\| c^{\sigma(2)}\left\|_{l_{p}^{1}}\right\| c^{\sigma(3)} \|_{l_{p}^{1}}
$$

As a warm up, we can prove the following simple estimates, which are sufficient for the local theory.

LEMMA 2.1. The following estimates hold:

$$
\begin{aligned}
\left\|\mathcal{N}^{t}[F, G, H]\right\|_{S} & \lesssim(1+|t|)^{-1}\|F\|_{S}\|G\|_{S}\|H\|_{S}, \\
\left\|\mathcal{N}^{t}\left[F^{a}, F^{b}, F^{c}\right]\right\|_{S^{+}} & \lesssim(1+|t|)^{-1} \max _{\sigma \in \mathfrak{S}_{3}}\left\|F^{\sigma(a)}\right\|_{S^{+}}\left\|F^{\sigma(b)}\right\|_{S}\left\|F^{\sigma(c)}\right\|_{S} .
\end{aligned}
$$

However, these estimates fall short of giving a satisfactory global theory.

Proof. Coming back to (2.5), we readily obtain

$$
\left\|\mathcal{I}^{t}\left[f^{a}, f^{b}, f^{c}\right]\right\|_{L_{x}^{2}} \lesssim \min _{\sigma \in \mathfrak{S}_{3}}\left\|f^{\sigma(a)}\right\|_{L_{x}^{2}}\left\|e^{i t \partial_{x x}} f^{\sigma(b)}\right\|_{L_{x}^{\infty}}\left\|e^{i t \partial_{x x}} f^{\sigma(c)}\right\|_{L_{x}^{\infty}}
$$

Assume that $|t| \geqslant 1$. We use the basic dispersive bound for the one-dimensional Schrödinger equation and (2.10) to get

$$
\left\|e^{i t \partial_{x x}} f\right\|_{L_{x}^{\infty}} \lesssim|t|^{-1 / 2}\|f\|_{L_{x}^{1}} \lesssim|t|^{-1 / 2}\|f\|_{L_{x}^{2}}^{1 / 2}\|x f\|_{L_{x}^{2}}^{1 / 2}
$$

Estimate (2.16) allows us to write, for any $\alpha>d$,

$$
\sum_{p \in \mathbb{Z}^{d}}\left\|e^{i t \partial_{x x}} F_{p}\right\|_{L_{x}^{\infty}} \lesssim|t|^{-1 / 2} \sum_{p \in \mathbb{Z}^{d}}\langle p\rangle^{-\alpha}\left\|\langle p\rangle^{2 \alpha} F_{p}\right\|_{L_{x}^{2}}^{1 / 2}\left\|x F_{p}\right\|_{L_{x}^{2}}^{1 / 2} \lesssim|t|^{-1 / 2}\|F\|_{S} .
$$


If $|t| \leqslant 1$, we use Sobolev estimates instead of (2.16), and get

$$
\sum_{p \in \mathbb{Z}^{d}}\left\|e^{i t \partial_{x x}} F_{p}\right\|_{L_{x}^{\infty}} \lesssim \sum_{p \in \mathbb{Z}^{d}}\left\|F_{p}\right\|_{H_{x}^{1}} \lesssim\|F\|_{S} .
$$

We now can come back to (2.15): recalling (2.4), and using (2.13), we get the bound

$$
\left\|\mathcal{N}^{t}\left[F^{a}, F^{b}, F^{c}\right]\right\|_{L_{x, y}^{2}} \lesssim(1+|t|)^{-1} \min _{\sigma \in \mathfrak{S}_{3}}\left\|F^{\sigma(a)}\right\|_{L_{x, y}^{2}}\left\|F^{\sigma(b)}\right\|_{S}\left\|F^{\sigma(c)}\right\|_{S} .
$$

Now we can use Lemma 7.4. This completes the proof of Lemma 2.1.

\section{Structure of the nonlinearity}

The purpose of this section is to extract the key effective interactions from the full nonlinearity in (1.1). We first decompose the nonlinearity as

$$
\mathcal{N}^{t}[F, G, H]=\frac{\pi}{t} \mathcal{R}[F, G, H]+\mathcal{E}^{t}[F, G, H],
$$

where $\mathcal{R}$ is given in (2.6). Our main result is the following.

Proposition 3.1. Assume that, for $T \geqslant 1, F, G, H: \mathbb{R} \rightarrow S$ satisfy

$$
\|F\|_{X_{T}}+\|G\|_{X_{T}}+\|H\|_{X_{T}} \leqslant 1 .
$$

Then, for $t \in[T / 4, T]$, we can write

$$
\mathcal{E}^{t}[F(t), G(t), H(t)]=\mathcal{E}_{1}(t)+\mathcal{E}_{2}(t),
$$

where the following bounds hold uniformly in $T \geqslant 1$ :

$$
\begin{aligned}
T^{-\delta} \| & \int_{\mathbb{R}} q_{T}(t) \mathcal{E}_{i}(t) d t \|_{S} \lesssim 1, \quad i=1,2, \\
& T^{1+\delta} \sup _{T / 4 \leqslant t \leqslant T}\left\|\mathcal{E}_{1}(t)\right\|_{Z} \lesssim 1, \\
& T^{1 / 10} \sup _{T / 4 \leqslant t \leqslant T}\left\|\mathcal{E}_{3}(t)\right\|_{S} \lesssim 1,
\end{aligned}
$$

where $\mathcal{E}_{2}(t)=\partial_{t} \mathcal{E}_{3}(t)$. Assuming in addition that

$$
\|F\|_{X_{T}^{+}}+\|G\|_{X_{T}^{+}}+\|H\|_{X_{T}^{+}} \leqslant 1
$$

we also have that

$$
T^{-5 \delta}\left\|\int_{\mathbb{R}} q_{T}(t) \mathcal{E}_{i}(t) d t\right\|_{S^{+}} \lesssim 1, \quad T^{2 \delta}\left\|\int_{\mathbb{R}} q_{T}(t) \mathcal{E}_{i}(t) d t\right\|_{S} \lesssim 1, \quad i=1,2 .
$$


We will give a proof of Proposition 3.1 at the end of this section. It depends on various lemmas that we prove first. Among these lemmas, Lemma 3.2, Lemma 3.3, and the first part of Lemma 3.7 are essentially based on $L^{2}$ arguments, while Lemma 3.6 and the second part of Lemma 3.7 are based on regularity in Fourier space.

3.1. The high-frequency estimates. We start with an estimate bounding high frequencies in $x$. It uses essentially the bilinear Strichartz estimates on $\mathbb{R}$ (see Lemma 7.2 and [28]).

Lemma 3.2. Assume that $T \geqslant 1$. The following estimates hold uniformly in $T$ :

$$
\begin{aligned}
& \left\|\sum_{\substack{A, B, C \\
\max (A, B, C) \geqslant T^{1 / 6}}} \mathcal{N}^{t}\left[Q_{A} F, Q_{B} G, Q_{C} H\right]\right\|_{Z} \\
& \lesssim T^{-7 / 6}\|F\|_{S}\|G\|_{S}\|H\|_{S} \quad \forall t \geqslant T / 4, \\
& \quad \sum_{\substack{A, B, C \\
\max (A, B, C) \geqslant T^{1 / 6}}} \int_{\mathbb{R}} q_{T}(t) \mathcal{N}^{t}\left[Q_{A} F(t), Q_{B} G(t), Q_{C} H(t)\right] d t \|_{S} \\
& \lesssim T^{-1 / 50}\|F\|_{X_{T}}\|G\|_{X_{T}}\|H\|_{X_{T}}, \\
& \sum_{\substack{A, B, C \\
\max (A, B, C) \geqslant T^{1 / 6}}}^{\lesssim T^{-1 / 50}\|F\|_{X_{T}^{+}}\|G\|_{X_{T}^{+}}\|H\|_{X_{T}^{+}} .}
\end{aligned}
$$

Proof. We start by proving the first inequality of Lemma 3.2. Fixing $t \geqslant T / 4$ and invoking the bound (2.11) and Lemma 2.1, we obtain that it suffices to prove the bound

$$
\left\|\sum_{\substack{A, B, C \\ \max (A, B, C) \geqslant T^{1 / 6}}} \mathcal{N}^{t}\left[Q_{A} F, Q_{B} G, Q_{C} H\right]\right\|_{L_{x, y}^{2}} \lesssim T^{-5 / 3}\|F\|_{S}\|G\|_{S}\|H\|_{S} .
$$

Coming back to (2.4), and using that $l_{p}^{1} \subset l_{p}^{2}$, we see that (3.6) follows from $\sum_{(p, q, r, s) \in \mathcal{M}} \sum_{\substack{A, B, C \\ \max (A, B, C) \geqslant T^{1 / 6}}}\left\|\mathcal{I}^{t}\left[Q_{A} F_{q}, Q_{B} G_{r}, Q_{C} H_{S}\right]\right\|_{L_{x}^{2}} \lesssim T^{-5 / 3}\|F\|_{S}\|G\|_{S}\|H\|_{S}$. 
Using (2.5) and the Sobolev embedding, we see that

$$
\begin{aligned}
& \left\|\mathcal{I}^{t}\left[Q_{A} F_{q}, Q_{B} G_{r}, Q_{C} H_{s}\right]\right\|_{L_{x}^{2}} \\
& \quad \lesssim(A B C)^{-11}\left\|Q_{A} F_{q}\right\|_{H_{x}^{12}}\left\|Q_{B} G_{r}\right\|_{H_{x}^{12}}\left\|Q_{C} H_{s}\right\|_{H_{x}^{12}} \\
& \quad \lesssim(A B C)^{-11}(\langle q\rangle\langle r\rangle\langle s\rangle)^{-d-1}\|F\|_{H_{x, y}^{13+d}}\|G\|_{H_{x, y}^{13+d}}\|H\|_{H_{x, y}^{13+d}}
\end{aligned}
$$

Summing, we complete the proof of the first inequality of Lemma 3.2.

Let us now turn to the proof of the two remaining estimates. We first note that, for every $t$ and every $F, G, H \in S$ (respectively $S^{+}$)

$$
\begin{aligned}
& \left\|\sum_{\substack{A, B, C \\
\operatorname{med}(A, B, C) \geqslant T^{1 / 6} / 16}} \mathcal{N}^{t}\left[Q_{A} F, Q_{B} G, Q_{C} H\right]\right\|_{S} \lesssim T^{-7 / 6}\|F\|_{S}\|G\|_{S}\|H\|_{S}, \\
& \sum_{\substack{A, B, C \\
\operatorname{med}(A, B, C) \geqslant T^{1 / 6} / 16}} \mathcal{N}^{t}\left[Q_{A} F, Q_{B} G, Q_{C} H\right]\left\|_{S^{+}} \lesssim T^{-7 / 6}\right\| F\left\|_{S^{+}}\right\| G\left\|_{S^{+}}\right\| H \|_{S^{+}},
\end{aligned}
$$

where $\operatorname{med}(A, B, C)$ means the second largest number between $A, B, C$. The proof of (3.7) is slightly more delicate than the first inequality of Lemma 3.2, because, in aiming to apply Lemma 7.4, we are not allowed to lose derivatives on at least one of the $F, G, H$. Let $K \in L_{x, p}^{2}$; then we need to bound

$$
\begin{aligned}
I_{K}= & \left\langle K, \sum_{\substack{A, B, C \\
\operatorname{med}(A, B, C) \geqslant T^{1 / 6} / 16}} \mathcal{N}^{t}\left[Q_{A} F, Q_{B} G, Q_{C} H\right]\right\rangle_{L_{x, p}^{2} \times L_{x, p}^{2}}\left|\sum_{(p, q, r, s) \in \mathcal{M}}\right| \int_{\substack{A, B, C \\
\operatorname{med}(A, B, C) \geqslant T^{1 / 6} / 16}} \mathcal{U}(t)\left(Q_{A} F_{q}\right) \cdot \overline{\mathcal{U}(t)\left(Q_{B} G_{r}\right)} \\
& \cdot \mathcal{U}(t)\left(Q_{C} H_{s}\right) \cdot \overline{\mathcal{U}(t) K_{p}} \mid .
\end{aligned}
$$

We will show that

$$
I_{K} \lesssim T^{-5 / 3}\|F\|_{L_{x, y}^{2}}\|K\|_{L_{x, p}^{2}}\|G\|_{S}\|H\|_{S} .
$$

Similar estimates hold with $F$ replaced by $G$ or $H$. By duality and Lemma 7.4, this is sufficient to prove (3.7).

By performing a Littlewood-Paley decomposition of $K_{p}$, and using Sobolev inequality, we see from (3.8) that

$$
I_{K} \lesssim \sum_{(p, q, r, s) \in \mathcal{M}} \sum_{*}(B C)^{-11}\left\|Q_{A} F_{q}\right\|_{L_{x}^{2}}\left\|Q_{B} G_{r}\right\|_{H_{x}^{12}}\left\|Q_{C} H_{s}\right\|_{H_{x}^{12}}\left\|Q_{D} K_{p}\right\|_{L_{x}^{2}}
$$


where $\sum_{*}$ denotes the sum over all dyadic integers $A, B, C, D$ such that the two highest are comparable, and, in addition, $\operatorname{med}(A, B, C) \geqslant T^{1 / 6}$. Now note that

$$
\begin{aligned}
& \sum_{*}(B C)^{-11}\left\|Q_{A} F_{q}\right\|_{L_{x}^{2}}\left\|Q_{B} G_{r}\right\|_{H_{x}^{12}}\left\|Q_{C} H_{s}\right\|_{H_{x}^{12}}\left\|Q_{D} K_{p}\right\|_{L_{x}^{2}} \\
& \quad \lesssim \sum_{*}(\operatorname{med}(A, B, C))^{-11}\left\|Q_{A} F_{q}\right\|_{L_{x}^{2}}\left\|Q_{B} G_{r}\right\|_{H_{x}^{12}}\left\|Q_{C} H_{s}\right\|_{H_{x}^{12}}\left\|Q_{D} K_{p}\right\|_{L_{x}^{2}} \\
& \quad \lesssim T^{-5 / 3}\left\|F_{q}\right\|_{L_{x}^{2}}\left\|K_{p}\right\|_{L_{x}^{2}}\left\|G_{r}\right\|_{H_{x}^{12}}\left\|H_{S}\right\|_{H_{x}^{12}},
\end{aligned}
$$

where, in the last inequality, we have crudely summed over the two smallest dyadic numbers and applied the Cauchy-Schwarz inequality on the two highest. Using Cauchy-Schwarz inequality again in $p, q$, we see from (3.10) that

$$
I_{K} \lesssim T^{-5 / 3}\|F\|_{L_{x, y}^{2}}\|K\|_{L_{x, p}^{2}}\left(\sum_{r}\left\|G_{r}\right\|_{H_{x}^{12}}\right)\left(\sum_{s}\left\|H_{s}\right\|_{H_{x}^{12}}\right),
$$

which yields (3.9) and thus (3.7).

It therefore remains to prove that

$$
\begin{aligned}
& \left\|\sum_{(A, B, C) \in \Lambda} \int_{\mathbb{R}} q_{T}(t) \mathcal{N}^{t}\left[Q_{A} F(t), Q_{B} G(t), Q_{C} H(t)\right] d t\right\|_{S} \\
& \quad \lesssim T^{-1 / 50}\|F\|_{X_{T}}\|G\|_{X_{T}}\|H\|_{X_{T}},
\end{aligned}
$$

and

$$
\begin{aligned}
& \left\|\sum_{(A, B, C) \in \Lambda} \int_{\mathbb{R}} q_{T}(t) \mathcal{N}^{t}\left[Q_{A} F(t), Q_{B} G(t), Q_{C} H(t)\right] d t\right\|_{S^{+}} \\
& \quad \lesssim T^{-1 / 50}\|F\|_{X_{T}^{+}}\|G\|_{X_{T}^{+}}\|H\|_{X_{T}^{+}},
\end{aligned}
$$

where $(A, B, C) \in \Lambda$ means that the $A, B, C$ summation ranges over $\operatorname{med}(A, B$, $C) \leqslant T^{1 / 6} / 16$ and $\max (A, B, C) \geqslant T^{1 / 6}$. We shall only give the proof of (3.11), the proof of (3.12) being similar.

We consider a decomposition

$$
[T / 4,2 T]=\bigcup_{j \in J} I_{j}, \quad I_{j}=\left[j T^{9 / 10},(j+1) T^{9 / 10}\right]=\left[t_{j}, t_{j+1}\right], \quad \# J \lesssim T^{1 / 10}
$$

and consider $\chi \in C_{c}^{\infty}(\mathbb{R}), \chi \geqslant 0$ such that $\chi(x)=0$ if $|x| \geqslant 2$ and

$$
\sum_{k \in \mathbb{Z}} \chi(x-k) \equiv 1
$$


The left-hand side of (3.11) can be estimated by $C\left(E_{1}+E_{2}\right)$, where

$$
\begin{aligned}
E_{1}= & \| \sum_{j \in J} \sum_{(A, B, C) \in \Lambda} \int_{\mathbb{R}} q_{T}(t) \chi\left(\frac{t}{T^{9 / 10}}-j\right)\left(\mathcal{N}^{t}\left[Q_{A} F(t), Q_{B} G(t), Q_{C} H(t)\right]\right. \\
& \left.-\mathcal{N}^{t}\left[Q_{A} F\left(t_{j}\right), Q_{B} G\left(t_{j}\right), Q_{C} H\left(t_{j}\right)\right]\right) d t \|_{S}
\end{aligned}
$$

and

$$
\begin{aligned}
E_{2}= & \| \sum_{j \in J} \sum_{(A, B, C) \in \Lambda} \int_{\mathbb{R}} q_{T}(t) \chi\left(\frac{t}{T^{9 / 10}}-j\right) \\
& \times \mathcal{N}^{t}\left[Q_{A} F\left(t_{j}\right), Q_{B} G\left(t_{j}\right), Q_{C} H\left(t_{j}\right)\right] d t \|_{S} .
\end{aligned}
$$

Let us now turn to the estimate for $E_{1}$. We can write

$$
E_{1} \leqslant \sum_{j \in J} \int_{\mathbb{R}} q_{T}(t) \chi\left(\frac{t}{T^{9 / 10}}-j\right) E_{1, j}(t) d t,
$$

where

$$
\begin{aligned}
E_{1, j}(t):= & \sum_{(A, B, C) \in \Lambda}\left(\mathcal{N}^{t}\left[Q_{A} F(t), Q_{B} G(t), Q_{C} H(t)\right]\right. \\
& \left.\quad-\mathcal{N}^{t}\left[Q_{A} F\left(t_{j}\right), Q_{B} G\left(t_{j}\right), Q_{C} H\left(t_{j}\right)\right]\right) \|_{S} .
\end{aligned}
$$

At this point, we note that

$$
\begin{aligned}
& \sum_{(A, B, C) \in \Lambda} \mathcal{N}^{t}\left[Q_{A} F, Q_{B} G, Q_{C} H\right]=\mathcal{N}^{t}\left[Q_{+} F, Q_{-} G, Q_{-} H\right] \\
& +\mathcal{N}^{t}\left[Q_{-} F, Q_{+} G, Q_{-} H\right]+\mathcal{N}^{t}\left[Q_{-} F, Q_{-} G, Q_{+} H\right] \\
& Q_{+}:=Q_{\geqslant T^{1 / 6}}, \quad Q_{-}:=Q_{\leqslant T^{1 / 6} / 16} \text {. }
\end{aligned}
$$

Therefore, using Lemma 2.1, and the boundedness of $Q_{ \pm}$on $S$, we see that

$$
\begin{aligned}
E_{1, j}(t) \leqslant & (1+|t|)^{-1}\left[\left\|F(t)-F\left(t_{j}\right)\right\|_{S}\|G(t)\|_{S}\|H(t)\|_{S}\right. \\
& +\left\|F\left(t_{j}\right)\right\|_{S}\left\|G(t)-G\left(t_{j}\right)\right\|_{S}\|H(t)\|_{S} \\
& \left.+\left\|F\left(t_{j}\right)\right\|_{S}\left\|G\left(t_{j}\right)\right\|_{S}\left\|H(t)-H\left(t_{j}\right)\right\|_{S}\right] .
\end{aligned}
$$


Since $\left|t-t_{j}\right| \leqslant T^{9 / 10}$, we see by definition (2.12) that

$$
\left\|F(t)-F\left(t_{j}\right)\right\|_{S} \leqslant \int_{t_{j}}^{t}\left\|\partial_{t} F(\sigma)\right\|_{S} d \sigma \lesssim T^{-(1 / 10)+3 \delta}\|F\|_{X_{T}}
$$

Similar bounds hold for $G$ and $H$. Therefore, we can bound (3.15) by

$$
E_{1, j}(t) \lesssim T^{-(11 / 10)+5 \delta}\|F\|_{X_{T}}\|G\|_{X_{T}}\|H\|_{X_{T}}
$$

which in view of (3.13) and (3.14) is more than enough to bound the contribution of $E_{1}$.

It therefore only remains to estimate $E_{2}$. In this case the $A, B, C$ summation will not cause any difficulty, since the bilinear Strichartz estimates will provide a decay in terms of $(\max (A, B, C))^{-1}$. We have that

$$
E_{2} \leqslant \sum_{j \in J} \sum_{(A, B, C) \in \Lambda} E_{2, j}^{A, B, C}
$$

where

$$
E_{2, j}^{A, B, C}=\left\|\int_{\mathbb{R}} q_{T}(t) \chi\left(\frac{t}{T^{9 / 10}}-j\right) \mathcal{N}^{t}\left[Q_{A} F\left(t_{j}\right), Q_{B} G\left(t_{j}\right), Q_{C} H\left(t_{j}\right)\right] d t\right\|_{S} .
$$

Note that the profiles $F\left(t_{j}\right), G\left(t_{j}\right), H\left(t_{j}\right)$ are fixed. Using Lemma 7.4, it suffices to show that

$$
\begin{aligned}
& \left\|\sum_{(p, q, r, s) \in \mathcal{M}} \int_{\mathbb{R}} q_{T}(t) \chi\left(\frac{t}{T^{9 / 10}}-j\right) \mathcal{I}^{t}\left[Q_{A} F_{q}^{a}, Q_{B} F_{r}^{b}, Q_{C} F_{s}^{c}\right] d t\right\|_{L_{x, p}^{2}} \\
& \lesssim(\max (A, B, C))^{-1} \min _{\sigma \in \mathfrak{S}_{3}}\left\|F^{\sigma(a)}\right\|_{L_{x, y}^{2}}\left\|F^{\sigma(b)}\right\|_{S}\left\|F^{\sigma(c)}\right\|_{S}
\end{aligned}
$$

Indeed,

$$
\begin{gathered}
\sum_{j \in J} \sum_{(A, B, C) \in \Lambda}(\max (A, B, C))^{-1} \lesssim T^{-1 / 20}, \\
\left\|F\left(t_{j}\right)\right\|_{S}\left\|G\left(t_{j}\right)\right\|_{S}\left\|H\left(t_{j}\right)\right\|_{S} \leqslant T^{3 \delta}\|F\|_{X_{T}}\|G\|_{X_{T}}\|H\|_{X_{T}} .
\end{gathered}
$$


We proceed by duality. Let $K \in L_{x, p}^{2}$. We consider

$$
\begin{aligned}
I_{K}= & \left\langle K, \sum_{(p, q, r, s) \in \mathcal{M}} \int_{\mathbb{R}} q_{T}(t) \chi\left(\frac{t}{T^{9 / 10}}-j\right) \mathcal{I}^{t}\left[Q_{A} F_{q}^{a}, Q_{B} F_{r}^{b}, Q_{C} F_{s}^{c}\right] d t\right\rangle_{L_{x, p}^{2} \times L_{x, p}^{2}} \\
= & \sum_{(p, q, r, s) \in \mathcal{M}} \int_{\mathbb{R}^{2}} q_{T}(t) \chi\left(\frac{t}{T^{9 / 10}}-j\right) \mathcal{U}(t)\left(Q_{A} F_{q}^{a}\right) \\
& \cdot \overline{\mathcal{U}(t)\left(Q_{B} F_{r}^{b}\right)} \cdot \mathcal{U}(t)\left(Q_{C} F_{s}^{c}\right) \cdot \overline{\mathcal{U}(t) K_{p}} d x d t,
\end{aligned}
$$

where we may assume that $K=Q_{D} K, D \simeq \max (A, B, C)$. Using Lemma 7.2, we can estimate

$$
I_{K} \leqslant \sum_{(p, q, r, s) \in \mathcal{M}} D^{-1}\left\|F_{q}^{a}\right\|_{L_{x}^{2}}\left\|F_{r}^{b}\right\|_{L_{x}^{2}}\left\|F_{s}^{c}\right\|_{L_{x}^{2}}\left\|K_{p}\right\|_{L_{x}^{2}} .
$$

We can now use (2.13) to evaluate the sum. By duality, this yields (3.16) and therefore (3.11). As already mentioned, the proof of (3.12) is similar. This completes the proof of Lemma 3.2.

At this point, we introduce a first decomposition,

$$
\begin{gathered}
\mathcal{N}^{t}[F, G, H]=\Pi^{t}[F, G, H]+\tilde{\mathcal{N}}^{t}[F, G, H], \\
\mathcal{F} \Pi^{t}[F, G, H](\xi, p):=\sum_{(p, q, r, s) \in \Gamma_{0}} \mathcal{I}^{t}\left[\widehat{F_{q}, G_{r}}, H_{s}\right](\xi) .
\end{gathered}
$$

The contribution of $\tilde{\mathcal{N}}$ is treated in Section 3.2, and that of $\Pi^{t}$ in Section 3.3.

3.2. The fast oscillations. The main purpose of this subsection is to prove the following.

LemmA 3.3. For $T \geqslant 1$, assume that $F, G, H: \mathbb{R} \rightarrow S$ satisfy (3.2) and

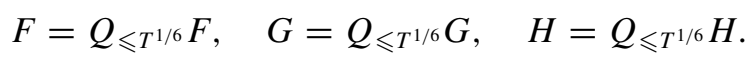

Then, for $t \in[T / 4, T]$, we can write

$$
\tilde{\mathcal{N}}^{t}[F(t), G(t), H(t)]=\widetilde{\mathcal{E}}_{1}^{t}+\mathcal{E}_{2}^{t},
$$

where it holds that, uniformly in $T \geqslant 1$,

$$
T^{1+2 \delta} \sup _{T / 4 \leqslant t \leqslant T}\left\|\widetilde{\mathcal{E}}_{1}(t)\right\|_{S} \lesssim 1, \quad T^{1 / 10} \sup _{T / 4 \leqslant t \leqslant T}\left\|\mathcal{E}_{3}(t)\right\|_{S} \lesssim 1,
$$


where $\mathcal{E}_{2}(t)=\partial_{t} \mathcal{E}_{3}(t)$. Assuming in addition that (3.4) holds, we have

$$
T^{1+2 \delta} \sup _{T / 4 \leqslant t \leqslant T}\left\|\widetilde{\mathcal{E}}_{1}(t)\right\|_{S^{+}} \lesssim 1, \quad T^{1 / 10} \sup _{T / 4 \leqslant t \leqslant T}\left\|\mathcal{E}_{3}(t)\right\|_{S^{+}} \lesssim 1 .
$$

To prove this lemma, we start by decomposing $\tilde{\mathcal{N}}^{t}$ along the nonresonant level sets as follows:

$$
\begin{aligned}
\mathcal{F} \tilde{\mathcal{N}}^{t}[F, G, H](\xi, p)= & \sum_{\omega \neq 0} \sum_{(p, q, r, s) \in \Gamma_{\omega}} e^{i t \omega}\left(\mathcal{O}_{1}^{t}\left[F_{q}, G_{r}, H_{s}\right](\xi)\right. \\
& \left.+\mathcal{O}_{2}^{t}\left[F_{q}, G_{r}, H_{s}\right](\xi)\right), \\
\mathcal{O}_{1}^{t}[f, g, h](\xi):= & \int_{\mathbb{R}^{2}} e^{2 i t \eta \kappa}\left(1-\varphi\left(t^{1 / 4} \eta \kappa\right)\right) \widehat{f}(\xi-\eta) \widehat{g}(\xi-\eta-\kappa) \\
& \times \widehat{h}(\xi-\kappa) d \eta d \kappa, \\
\mathcal{O}_{2}^{t}[f, g, h](\xi):= & \int_{\mathbb{R}^{2}} e^{2 i t \eta \kappa} \varphi\left(t^{1 / 4} \eta \kappa\right) \widehat{f}(\xi-\eta) \widehat{g}(\xi-\eta-\kappa) \\
& \times \widehat{h}(\xi-\kappa) d \eta d \kappa .
\end{aligned}
$$

Essentially, on $\mathcal{O}_{1}$, we use the fact that the interactions are noncoherent (in the terminology of Germain-Masmoudi-Shatah [42], $\mathcal{O}_{1}$ corresponds to space nonresonant interactions and $\mathcal{O}_{2}$ to time nonresonant interactions) (see Lemma 3.6), while, on $\mathcal{O}_{2}$, we exploit the fact that they are nonresonant, and we can use a normal forms transformation.

Before we go into the proof of Lemma 3.3, we make the following remarks.

REMARK 3.4. Some of our estimates below will concern functions of one real variable. To pass them on to functions on $\mathbb{R} \times \mathbb{T}^{d}$, we define

$$
\|f\|_{Y}:=\left\|\langle x\rangle^{9 / 10} f\right\|_{L_{x}^{2}}+\|f\|_{H_{x}^{3 N / 4}}
$$

and use that

$$
\sum_{p \in \mathbb{Z}^{d}}\left\|F_{p}\right\|_{Y} \lesssim\|F\|_{S} .
$$

REMARK 3.5. Assume that $T / 4 \leqslant t \leqslant T$. Under the assumptions of Lemma 3.3, the multiplier appearing in the definition of $\mathcal{O}_{2}^{t}$ in (3.18) can be taken to be

$$
\tilde{m}(\eta, \kappa):=\varphi\left(t^{1 / 4} \eta \kappa\right) \varphi\left((10 T)^{-1 / 6} \eta\right) \varphi\left((10 T)^{-1 / 6} \kappa\right) .
$$

We note that

$$
\left\|\mathcal{F}_{\eta \kappa} \tilde{m}\right\|_{L^{1}\left(\mathbb{R}^{2}\right)}=\left\|I\left(x_{1}, x_{2}\right)\right\|_{L_{x_{1}, x_{2}}^{1}},
$$


where

$$
I\left(x_{1}, x_{2}\right)=\int_{\mathbb{R}^{2}} e^{i x_{1} \eta} e^{i x_{2} \kappa} \varphi(S \eta \kappa) \varphi(\eta) \varphi(\kappa) d \eta d \kappa, \quad S \approx T^{7 / 12}
$$

Then one may show that

$$
\left|I\left(x_{1}, x_{2}\right)\right|+\left|x_{1} I\left(x_{1}, x_{2}\right)\right|+\left|x_{2} I\left(x_{1}, x_{2}\right)\right| \lesssim 1, \quad\left|x_{1} x_{2} I\left(x_{1}, x_{2}\right)\right| \lesssim \log (1+T) .
$$

One also has rough polynomial-in- $T$ bounds for $\left(x_{1}^{2}+x_{2}^{2}+x_{1}^{2} x_{2}^{2}\right)\left|I\left(x_{1}, x_{2}\right)\right|$. Therefore by interpolation one obtains that for every $\varepsilon>0$ there exists $\kappa>1$ such that

$$
\left|I\left(x_{1}, x_{2}\right)\right| \lesssim(1+T)^{\varepsilon}\left(1+x_{1}^{2}+x_{2}^{2}\right)^{-\kappa} .
$$

We hence deduce that $\left\|\mathcal{F}_{\eta \kappa} \tilde{m}\right\|_{L^{1}\left(\mathbb{R}^{2}\right)} \lesssim t^{\delta / 100}$. Applying Lemma 7.5 , we arrive at the following conclusion: if

$$
f^{a}=Q_{\leqslant T^{1 / 6}} f^{a}, \quad f^{b}=Q_{\leqslant T^{1 / 6}} f^{b}, \quad f^{c}=Q_{\leqslant T^{1 / 6}} f^{c},
$$

$t \geqslant T / 4$, then

$$
\begin{aligned}
\left\|\mathcal{O}_{2}^{t}\left[f^{a}, f^{b}, f^{c}\right]\right\|_{L_{\xi}^{2}} & =\left\|\mathcal{F} \mathcal{O}_{2}^{t}\left[f^{a}, f^{b}, f^{c}\right]\right\|_{L_{x}^{2}} \\
& \lesssim(1+|t|)^{\delta / 100} \min _{\sigma \in \mathfrak{S}_{3}}\left\|f^{\sigma(a)}\right\|_{L_{x}^{2}}\left\|e^{i t \partial_{x x}} f^{\sigma(b)}\right\|_{L_{x}^{\infty}}\left\|e^{i t \partial_{x x}} f^{\sigma(c)}\right\|_{L_{x}^{\infty}} \\
& \lesssim(1+|t|)^{-1+(\delta / 100)} \min _{\sigma \in \mathfrak{S}_{3}}\left\|f^{\sigma(a)}\right\|_{L_{x}^{2}}\left\|f^{\sigma(b)}\right\|_{Y}\left\|f^{\sigma(c)}\right\|_{Y} \cdot(3.20)
\end{aligned}
$$

A similar bound holds for $\mathcal{O}_{1}^{t}$ because $\mathcal{O}_{1}^{t}+\mathcal{O}_{2}^{t}$ enjoys a bound better than (3.20).

The contribution of $\mathcal{O}_{1}^{t}$ essentially follows from the following independent estimate. We now give the argument to bound the operator $\mathcal{O}_{1}^{t}$.

LEMMA 3.6. Assume that $t, f^{a}, f^{b}, f^{c}$ satisfy

$t \geqslant T / 4, \quad f^{a}=Q_{A} f^{a}, \quad f^{b}=Q_{B} f^{b}, \quad f^{c}=Q_{C} f^{c}, \quad \max (A, B, C) \leqslant T^{1 / 6}$.

Then

$$
\left\|\mathcal{O}_{1}^{t}\left[f^{a}, f^{b}, f^{c}\right]\right\|_{L_{\xi}^{2}} \lesssim T^{-201 / 200} \min _{\sigma \in \mathfrak{S}_{3}}\left\|f^{\sigma(a)}\right\|_{L_{x}^{2}}\left\|f^{\sigma(b)}\right\|_{Y}\left\|f^{\sigma(c)}\right\|_{Y} .
$$

Proof. We will show that

$$
\left\|\mathcal{O}_{1}^{t}[f, g, h]\right\|_{L_{\xi}^{2}} \lesssim T^{-201 / 200}\|f\|_{L_{x}^{2}}\|g\|_{Y}\|h\|_{Y}
$$

The other inequalities in (3.21) follow by symmetry and conjugation. 
We first decompose

$$
\begin{gathered}
g=g_{c}+g_{f}, \quad h=h_{c}+h_{f}, \quad g_{c}(x)=\varphi\left(\frac{x}{D}\right) g(x), \\
h_{c}(x)=\varphi\left(\frac{x}{D}\right) h(x), \quad D:=T^{(7 / 12)-(\delta / 10)} .
\end{gathered}
$$

Using the remark after (3.20), we see that

$$
\left\|\mathcal{O}_{1}^{t}[f, g, h]\right\|_{L_{\xi}^{2}} \lesssim t^{\delta / 100}\|f\|_{L_{x}^{2}}\left\|e^{i t \partial_{x x}} g\right\|_{L_{x}^{\infty}}\left\|e^{i t \partial_{x x}} h\right\|_{L_{x}^{\infty}} .
$$

In addition, for $\gamma>\operatorname{frac} 12$,

$$
\left\|e^{i t \partial_{x x}} f\right\|_{L_{x}^{\infty}} \lesssim\langle t\rangle^{-1 / 2}\|f\|_{L_{x}^{1}}=\langle t\rangle^{-1 / 2}\left\|\langle x\rangle^{\gamma}\langle x\rangle^{-\gamma} f\right\|_{L_{x}^{1}} \lesssim\langle t\rangle^{-1 / 2}\left\|\langle x\rangle^{\gamma} f\right\|_{L_{x}^{2}} .
$$

Hence, if $f(x)$ is supported in $|x|>R$,

$$
\left\|e^{i t \partial_{x x}} f\right\|_{L_{x}^{\infty}} \lesssim\langle t\rangle^{-1 / 2} R^{-\alpha}\|f\|_{Y}
$$

with $\alpha>\frac{2}{5}$. Therefore we obtain that (3.22) is a consequence of the estimate

$$
\left\|\mathcal{O}_{1}^{t}\left[f, g_{c}, h_{c}\right]\right\|_{L_{\xi}^{2}} \lesssim T^{-20}\|f\|_{L_{x}^{2}}\|g\|_{L_{x}^{2}}\|h\|_{L_{x}^{2}} .
$$

But this follows by repeated integration by parts in $\kappa$ since, on the support of integration, we necessarily have $|\eta| \gtrsim T^{-5 / 12}$. This completes the proof of Lemma 3.6.

We can now proceed to the proof of Lemma 3.3.

Proof of Lemma 3.3. In the decomposition of $\tilde{\mathcal{N}}$ in (3.18), the first sum involving $\mathcal{O}_{1}^{t}$ contributes to $\widetilde{\mathcal{E}}_{1}(t)$, and its estimate follows by combining (3.19), Lemma 3.6, and Lemma 7.4. Indeed, from (3.21), (2.13), and Remark 3.4, we get that, for $t \geqslant T / 4$,

$$
\begin{aligned}
& \left\|\sum_{\omega \neq 0} \sum_{(p, q, r, s) \in \Gamma_{\omega}} e^{i t \omega} \mathcal{O}_{1}^{t}\left[F_{q}^{a}, F_{r}^{b}, F_{s}^{c}\right]\right\|_{L_{\xi, p}^{2}} \leqslant T^{-201 / 200} \min _{\sigma \in \mathfrak{S}_{3}}\left\|F^{\sigma(a)}\right\|_{L_{x, y}^{2}}\left\|F^{\sigma(b)}\right\|_{S}\left\|F^{\sigma(c)}\right\|_{S} .
\end{aligned}
$$

Lemma 7.4 (with $\theta_{1}=1$ and $\theta_{2}=0$, and $K=T^{-1-(1 / 200)} \lesssim T^{-1-5 \delta}$ ) then gives the result.

We now consider the contribution of the second sum in (3.18). We start with a simple observation. Defining

$$
\widetilde{\mathcal{O}}_{2, \omega}^{t}[F, G, H](\xi, p)=\sum_{(p, q, r, s) \in \Gamma_{\omega}} \mathcal{O}_{2}^{t}\left[F_{q}, G_{r}, H_{s}\right](\xi),
$$


it follows from (3.20) that, for $K \in L_{\xi, p}^{2}\left(\mathbb{R} \times \mathbb{Z}^{d}\right)$,

$$
\begin{aligned}
& \left\langle K, \widetilde{\mathcal{O}}_{2, \omega}^{t}[F, G, H]\right\rangle_{L_{\xi, p}^{2} \times L_{\xi, p}^{2}} \\
& \leqslant \sum_{(p, q, r, s) \in \Gamma_{\omega}}\left|\left\langle K_{p}, \mathcal{O}_{2}^{t}\left[F_{q}, G_{r}, H_{S}\right]\right\rangle_{L_{\xi}^{2} \times L_{\xi}^{2}}\right| \lesssim(1+|t|)^{-1+\delta} \sum_{(p, q, r, s) \in \Gamma_{\omega}}\left\|K_{p}\right\|_{L_{\xi}^{2}} \\
& \quad \times \min \left\{\left\|F_{q}\right\|_{L_{x}^{2}}\left\|G_{r}\right\|_{Y}\left\|H_{s}\right\|_{Y},\left\|F_{q}\right\|_{Y}\left\|G_{r}\right\|_{L_{x}^{2}}\left\|H_{s}\right\|_{Y},\left\|F_{q}\right\|_{Y}\left\|G_{r}\right\|_{Y}\left\|H_{s}\right\|_{L_{x}^{2}}\right\},
\end{aligned}
$$

and summing over $\omega$, using (3.19) and (2.13), we get

$$
\left\|\sum_{\omega} e^{i t \omega} \widetilde{\mathcal{O}}_{2, \omega}^{t}\left[F^{a}, F^{b}, F^{c}\right]\right\|_{L_{\xi, p}^{2}} \lesssim(1+|t|)^{-1+\delta} \min _{\sigma \in \mathfrak{S}_{3}}\left\|F^{\sigma(a)}\right\|_{L_{x, y}^{2}}\left\|F^{\sigma(b)}\right\|_{S}\left\|F^{\sigma(c)}\right\|_{S} .
$$

Now observe that

$$
\begin{aligned}
e^{i t \omega} \mathcal{O}_{2}^{t}[f, g, h]= & \partial_{t}\left(\frac{e^{i t \omega}}{i \omega} \mathcal{O}_{2}^{t}[f, g, h]\right)-e^{i t \omega}\left(\partial_{t} \mathcal{O}_{2}^{t}\right)[f, g, h] \\
& -e^{i t \omega} \mathcal{O}_{2}^{t}\left[\partial_{t} f, g, h\right]-e^{i t \omega} \mathcal{O}_{2}^{t}\left[f, \partial_{t} g, h\right]-e^{i t \omega} \mathcal{O}_{2}^{t}\left[f, g, \partial_{t} h\right],
\end{aligned}
$$

where

$\left(\partial_{t} \mathcal{O}_{2}^{t}\right)[f, g, h](\xi):=\int_{\mathbb{R}} \partial_{t}\left(e^{2 i t \eta \kappa} \varphi\left(t^{1 / 4} \eta \kappa\right)\right) \widehat{f}(\xi-\eta) \widehat{\widehat{g}}(\xi-\eta-\kappa) \widehat{h}(\xi-\kappa) d \eta d \kappa$.

We use here the fact $\partial_{t} f, \partial_{t} g$, and $\partial_{t} g$ have better decay than $f, g$, and $h$, which ultimately comes from our choice of unknowns as pullbacks of nonlinear solutions by the linear flow. Using (3.24), the definition of the $X_{T}$ norm, and Lemma 7.4, we see that the contribution of the second line in (3.25) is acceptable. Similarly, since $(1+|t|)^{1 / 4}\left(\partial_{t} \mathcal{O}_{2}^{t}\right)$ satisfies similar estimates as $\mathcal{O}_{2}^{t}$, the second term in the right-hand side of (3.25) is acceptable. It remains to analyze the first one. We define $\mathcal{E}_{3}$ by

$$
\mathcal{F E}_{3}(\xi, p):=\sum_{\omega \neq 0} \sum_{(p, q, r, s) \in \Gamma_{\omega}} \frac{e^{i t \omega}}{i \omega} \mathcal{O}_{2}^{t}\left[F_{q}, G_{r}, H_{s}\right](\xi) .
$$

Using (3.23) and (2.13), we see that, for $K \in L_{x, y}^{2}\left(\mathbb{R} \times \mathbb{T}^{d}\right)$,

$$
\begin{aligned}
& \left\langle K, \mathcal{E}_{3}\right\rangle_{L_{x, y}^{2} \times L_{x, y}^{2}} \\
& \leqslant \sum_{\omega \neq 0}\left|\left\langle\mathcal{F} K, \widetilde{\mathcal{O}}_{2, \omega}^{t}[F, G, H]\right\rangle_{L^{2} \times L^{2}}\right|, \lesssim(1+|t|)^{-1+\delta} \sum_{\omega \neq 0} \sum_{(p, q, r, s) \in \Gamma_{\omega}}\left\|\widehat{K}_{p}\right\|_{L_{\xi}^{2}}
\end{aligned}
$$




$$
\begin{aligned}
& \times \min \left\{\left\|F_{q}\right\|_{L_{x}^{2}}\left\|G_{r}\right\|_{Y}\left\|H_{S}\right\|_{Y},\left\|F_{q}\right\|_{Y}\left\|G_{r}\right\|_{L_{x}^{2}}\left\|H_{S}\right\|_{Y},\left\|F_{q}\right\|_{Y}\left\|G_{r}\right\|_{Y}\left\|H_{S}\right\|_{L_{x}^{2}}\right\} \\
\lesssim & (1+|t|)^{-1+\delta}\|K\|_{L_{x, y}^{2}} \\
& \times \min \left\{\|F\|_{L_{x, y}^{2}}\|G\|_{S}\|H\|_{S},\|F\|_{S}\|G\|_{L_{x, y}^{2}}\|H\|_{S},\|F\|_{S}\|G\|_{S}\|H\|_{L_{x, y}^{2}}\right\} .
\end{aligned}
$$

Another application of Lemma 7.4 shows that this term in the right-hand side of (3.25) gives an acceptable contribution.

3.3. The resonant level set. We now turn to the contribution of the resonant set in (3.17),

$$
\mathcal{F} \Pi^{t}[F, G, H](\xi, p)=\sum_{(p, q, r, s) \in \Gamma_{0}} \mathcal{F}_{x} \mathcal{I}^{t}\left[F_{q}(t), G_{r}(t), H_{s}(t)\right](\xi) .
$$

This term yields the main contribution in Proposition 3.1, and in particular is responsible for the slowest $1 / t$ decay. We show that it gives rise to a contribution which grows slowly in $S, S^{+}$, and that it can be well approximated by the resonant system in the $Z$-norm.

In this subsection, we will bound quantities in terms of

$$
\|F\|_{\tilde{Z}_{t}}:=\|F\|_{Z}+(1+|t|)^{-\delta}\|F\|_{S},
$$

so that $F(t)$ remains uniformly bounded in $\tilde{Z}_{t}$ under the assumption of Proposition 3.1.

LEMMA 3.7. Let $t \geqslant 1$. It holds that

$$
\left\|\Pi^{t}\left[F^{a}, F^{b}, F^{c}\right]\right\|_{S} \lesssim(1+|t|)^{-1} \sum_{\sigma \in \mathfrak{S}_{3}}\left\|F^{\sigma(a)}\right\|_{\tilde{Z}_{t}} \cdot\left\|F^{\sigma(b)}\right\|_{\tilde{Z}_{t}} \cdot\left\|F^{\sigma(c)}\right\|_{S}
$$

and

$$
\begin{aligned}
\left\|\Pi^{t}\left[F^{a}, F^{b}, F^{c}\right]\right\|_{S^{+}} \lesssim & (1+|t|)^{-1} \sum_{\sigma \in \mathfrak{S}_{3}}\left\|F^{\sigma(a)}\right\|_{\tilde{Z}_{t}} \cdot\left\|F^{\sigma(b)}\right\|_{\tilde{Z}_{t}} \cdot\left\|F^{\sigma(c)}\right\|_{S^{+}} \\
& +(1+|t|)^{-1+2 \delta} \sum_{\sigma \in \mathfrak{S}_{3}}\left\|F^{\sigma(a)}\right\|_{\tilde{Z}_{t}} \cdot\left\|F^{\sigma(b)}\right\|_{S} \cdot\left\|F^{\sigma(c)}\right\|_{S} .
\end{aligned}
$$

In addition,

$$
\left\|\Pi^{t}[F, G, H]-\frac{\pi}{t} \mathcal{R}[F, G, H]\right\|_{Z} \lesssim(1+|t|)^{-1-20 \delta}\|F\|_{S}\|G\|_{S}\|H\|_{S} .
$$

and

$$
\left\|\Pi^{t}[F, G, H]-\frac{\pi}{t} \mathcal{R}[F, G, H]\right\|_{S} \lesssim(1+|t|)^{-1-20 \delta}\|F\|_{S^{+}}\|G\|_{S^{+}}\|H\|_{S^{+}} .
$$


REMARK 3.8. Using Lemmas 7.1 and 7.4, we directly see that (3.26) and (3.27) also hold if $\Pi^{t}\left[F^{a}, F^{b}, F^{c}\right]$ is replaced by $(1+t)^{-1} \mathcal{R}\left[F^{a}, F^{b}, F^{c}\right]$.

REMARK 3.9. Note that, in Lemma 3.7, the summation in $p$ is a highly nontrivial part of the estimate, as opposed to the previous lemmas, which were essentially concerned with functions of a real variable, and the summation in $p$ was treated in a crude way via (2.13).

The proof of Lemma 3.7 relies on the following key lemma, which essentially allows us to extract the resonant interaction for fixed periodic frequencies. The proof is essentially an adaptation of [66] to our context.

LEMMA 3.10. Assume that

$$
f(x)=\varphi\left(s^{-1 / 4} x\right) f(x), \quad g(x)=\varphi\left(s^{-1 / 4} x\right) g(x), \quad h(x)=\varphi\left(s^{-1 / 4} x\right) h(x)
$$

and that $s \geqslant 1$. It holds that

$$
\begin{aligned}
& \left|\int_{\mathbb{R}^{2}} e^{i 2 s \eta \kappa} \widehat{f}(\xi-\eta) \overline{\widehat{g}}(\xi-\eta-\kappa) \widehat{h}(\xi-\kappa) d \eta d \kappa-\frac{\pi}{s} \widehat{f}(\xi) \overline{\widehat{g}}(\xi) \widehat{h}(\xi)\right| \\
& \quad \lesssim s^{-11 / 10}\|f\|_{L_{x}^{2}}\|g\|_{L_{x}^{2}}\|h\|_{L_{x}^{2}} .
\end{aligned}
$$

In fact, for $\theta$ an integer,

$$
\begin{aligned}
& |\xi|^{\theta}\left|\int_{\mathbb{R}^{2}} e^{i 2 s \eta \kappa} \widehat{f^{a}}(\xi-\eta) \widehat{\widehat{f^{b}}}(\xi-\eta-\kappa) \widehat{f}^{c}(\xi-\kappa) d \eta d \kappa-\frac{\pi}{s} \widehat{f^{a}}(\xi) \widehat{\widehat{f^{b}}}(\xi) \widehat{f^{c}}(\xi)\right| \\
& \quad \lesssim s^{-11 / 10} \min _{\sigma \in \mathfrak{S}_{3}}\left\|f^{\sigma(a)}\right\|_{H_{x}^{\theta}}\left\|f^{\sigma(b)}\right\|_{L_{x}^{2}}\left\|f^{\sigma(c)}\right\|_{L_{x}^{2}} .
\end{aligned}
$$

Proof of Lemma 3.10. We may rewrite the integral in equation (3.31) as

$$
\begin{aligned}
\int_{\mathbb{R}^{2}} & e^{i 2 s \eta \kappa} \widehat{f}(\xi-\eta) \widehat{g}(\xi-\eta-\kappa) \widehat{h}(\xi-\kappa) d \eta d \kappa \\
= & \int_{\mathbb{R}^{3}} f\left(y_{1}\right) \bar{g}\left(y_{2}\right) h\left(y_{3}\right) \int_{\mathbb{R}^{2}} e^{i\left[2 s \eta \kappa-y_{1}(\xi-\eta)-y_{2}(\eta+\kappa-\xi)-y_{3}(\xi-\kappa)\right]} d \eta d \kappa d y_{1} d y_{2} d y_{3} \\
= & \frac{1}{2 s} \int_{\mathbb{R}^{3}} f\left(y_{1}\right) \bar{g}\left(y_{2}\right) h\left(y_{3}\right) e^{-i \xi\left(y_{1}-y_{2}+y_{3}\right)} e^{-i\left(\left(y_{1}-y_{2}\right) / \sqrt{2 s}\right)\left(\left(y_{3}-y_{2}\right) / \sqrt{2 s}\right)} \\
& \times\left\{\int_{\mathbb{R}^{2}} e^{i\left[\eta+\left(\left(y_{3}-y_{2}\right) / \sqrt{2 s}\right)\right] \cdot\left[\kappa+\left(\left(y_{1}-y_{2}\right) / \sqrt{2 s}\right)\right]} d \eta d \kappa\right\} d y_{1} d y_{2} d y_{3} \\
= & \frac{\pi}{s} \int_{\mathbb{R}^{3}} f\left(y_{1}\right) \bar{g}\left(y_{2}\right) h\left(y_{3}\right) e^{-i \xi\left(y_{1}-y_{2}+y_{3}\right)} e^{-i\left(\left(y_{1}-y_{2}\right) / \sqrt{2 s}\right)\left(\left(y_{3}-y_{2}\right) / \sqrt{2 s}\right)} d y_{1} d y_{2} d y_{3} .
\end{aligned}
$$


Therefore, for $\xi \in \mathbb{R}$,

$$
\begin{aligned}
& \left|\int_{\mathbb{R}^{2}} e^{i 2 s \eta \kappa} \widehat{f}(\xi-\eta) \widehat{g}(\xi-\eta-\kappa) \widehat{h}(\xi-\kappa) d \eta d \kappa-\frac{\pi}{s} \widehat{f}(\xi) \widehat{g}(\xi) \widehat{h}(\xi)\right| \\
& \leqslant \mid \frac{\pi}{s} \int_{\mathbb{R}^{3}} f\left(y_{1}\right) \bar{g}\left(y_{2}\right) h\left(y_{3}\right) e^{-i \xi\left(y_{1}-y_{2}+y_{3}\right)} \\
& \quad \times\left\{e^{-i\left(\left(y_{1}-y_{2}\right) / \sqrt{2 s}\right)\left(\left(y_{3}-y_{2}\right) / \sqrt{2 s}\right)}-1\right\} d y_{1} d y_{2} d y_{3} \mid \\
& \quad \lesssim s^{-11 / 10}\|f\|_{L_{x}^{2}}\|g\|_{L_{x}^{2}}\|h\|_{L_{x}^{2}} .
\end{aligned}
$$

This concludes the proof of (3.31).

Now, (3.32) follows from (3.31) and the fact that

$$
\begin{aligned}
& \left|\int_{\mathbb{R}^{2}} e^{i 2 s \eta \kappa} \widehat{f^{a}}(\xi-\eta) \widehat{\widehat{f^{b}}}(\xi-\eta-\kappa) \widehat{f}^{c}(\xi-\kappa)\left(\kappa^{\alpha} \eta^{\beta}\right) d \eta d \kappa\right| \\
& \quad \lesssim s^{-(3 / 4)(\alpha+\beta)} \min _{\sigma \in \mathfrak{S}_{3}}\left\|\widehat{f}^{\sigma(a)}\right\|_{L_{x}^{1}}\left\|\widehat{f}^{\sigma(b)}\right\|_{L_{x}^{2}}\left\|\widehat{f}^{\sigma(c)}\right\|_{L_{x}^{2}},
\end{aligned}
$$

which is readily verified upon integrating by parts in $\eta$ and $\kappa$. This completes the proof of Lemma 3.10.

We are now ready to provide the proof of Lemma 3.7.

Proof of Lemma 3.7. Combining (2.5) with Lemma 7.1, we see that

$$
\begin{aligned}
& \left\|\Pi^{t}\left[F^{a}, F^{b}, F^{c}\right]\right\|_{L_{x, y}^{2}} \\
& \leqslant\left\|\sum_{(p, q, r, s) \in \Gamma_{0}}\left|e^{i t \partial_{x x}} F_{q}^{a}(x)\right| \cdot\left|e^{-i t \partial_{x x}} F_{r}^{b}(x)\right| \cdot\left|e^{i t \partial_{x x}} F_{s}^{c}(x)\right|\right\|_{l_{p}^{2} L_{x}^{2}} \\
& \quad \lesssim \min _{j \in\{a, b, c\}}\|\| e^{i t \partial_{x x}} F_{p}^{j}(x)\left\|_{l_{p}^{2}} \prod_{k \neq j}\left[\sum_{p \in \mathbb{Z}^{d}}\left[1+|p|^{2}\right]\left|e^{i t \partial_{x x}} F_{p}^{k}(x)\right|^{2}\right]^{1 / 2}\right\|_{L_{x}^{2}},
\end{aligned}
$$

and therefore

$$
\left\|\Pi^{t}\left[F^{a}, F^{b}, F^{c}\right]\right\|_{L_{x, y}^{2}} \lesssim \min _{j \in\{a, b, c\}}\left\|F^{j}\right\|_{L_{x, y}^{2}} \prod_{k \neq j}\left[\sup _{x \in \mathbb{R}} \sum_{p \in \mathbb{Z}^{d}}\left[1+|p|^{2}\right]\left|e^{i t \partial_{x x}} F_{p}^{k}(x)\right|^{2}\right]^{1 / 2} .
$$

Using Lemma 7.3, we can conclude that

$$
\left\|\Pi^{t}\left[F^{a}, F^{b}, F^{c}\right]\right\|_{L_{x, y}^{2}} \lesssim(1+|t|)^{-1} \min _{j \in\{a, b, c\}}\left\|F^{j}\right\|_{L_{x, y}^{2}} \prod_{k \neq j}\left\|F^{k}\right\|_{\tilde{Z}_{t}} .
$$


Using Lemma 7.4, we obtain (3.26). In order to show (3.27), we will apply the second part of Lemma 7.4. For this, it suffices to prove that

$$
\|x F\|_{Z} \lesssim T^{-\delta}\|F\|_{S^{+}}+T^{2 \delta}\|F\|_{S} .
$$

Indeed, one first observes that it suffices to prove (3.34) for functions independent of $y$. Then, we notice that

$$
\sup _{\xi}\left[\left(1+|\xi|^{2}\right)|\mathcal{F}(x f)|\right] \sim \sup _{M}\left(1+M^{2}\right)\left\|\mathcal{F} Q_{M}(x f)\right\|_{L_{\xi}^{\infty}} .
$$

Next, for every $M, R$ we get

$$
\begin{aligned}
\left\|\mathcal{F} Q_{M}[x(1-\varphi(x / R)) f]\right\|_{L_{\xi}^{\infty}} & \lesssim\|[x(1-\varphi(x / R)) f]\|_{L_{x}^{1}} \lesssim R^{-1 / 2}\left\|x^{2} f\right\|_{L^{2}} \\
& \leqslant C R^{-1 / 2}\|f\|_{S^{+}} .
\end{aligned}
$$

On the other hand, by invoking (2.10), we get

$$
\left\|\mathcal{F} Q_{M}[(x \varphi(x / R)) f]\right\|_{L_{\xi}^{\infty}} \lesssim\left\|Q_{M}(x(\varphi(x / R)) f)\right\|_{L_{x}^{2}}^{1 / 2}\left\|x Q_{M}[x(\varphi(x / R)) f]\right\|_{L_{x}^{2}}^{1 / 2} .
$$

We now estimate each factor at the right-hand side of the last inequality. By setting $\tilde{\varphi}(x)=x \varphi(x)$, we may write for $M$ a dyadic integer

$$
\left\|Q_{M}(x(\varphi(x / R)) f)\right\|_{L_{x}^{2}}=R\left\|Q_{M}((\tilde{\varphi}(x / R)) f)\right\|_{L_{x}^{2}} \lesssim R M^{-N}\|f\|_{H_{x}^{N}} .
$$

We next estimate the second factor in the right-hand side of (3.35) as follows:

$$
\left.\left\|x Q_{M}[x(\varphi(x / R)) f]\right\|_{L_{x}^{2}} \lesssim \|\langle x\rangle^{2} \varphi(x / R)\right) f\left\|_{L_{x}^{2}} \lesssim R\right\| f \|_{S} .
$$

We conclude the proof of (3.34) by choosing $R=T^{2 \delta}\left(1+M^{2}\right)^{2}$.

We now turn to the proof of (3.28) and (3.29). First decompose

$$
F=F_{c}+F_{f}, \quad G=G_{c}+G_{f}, \quad H=H_{c}+H_{f},
$$

where

$$
\begin{gathered}
F_{c}(x, y)=\varphi\left(t^{-1 / 4} x\right) F(x, y), \quad G_{c}(x, y)=\varphi\left(t^{-1 / 4} x\right) G(x, y), \\
H_{c}(x, y)=\varphi\left(t^{-1 / 4} x\right) H(x, y) .
\end{gathered}
$$

We claim that

$$
\begin{aligned}
& \left\|\Pi^{t}[F, G, H]-\Pi^{t}\left[F_{c}, G_{c}, H_{c}\right]\right\|_{Z}+\frac{1}{t}\left\|\mathcal{R}[F, G, H]-\mathcal{R}\left[F_{c}, G_{c}, H_{c}\right]\right\|_{Z} \\
& \quad \lesssim(1+|t|)^{-21 / 20}\|F\|_{S}\|G\|_{S}\|H\|_{S}
\end{aligned}
$$


and

$$
\begin{aligned}
& \left\|\Pi^{t}[F, G, H]-\Pi^{t}\left[F_{c}, G_{c}, H_{c}\right]\right\|_{S}+\frac{1}{t}\left\|\mathcal{R}[F, G, H]-\mathcal{R}\left[F_{c}, G_{c}, H_{c}\right]\right\|_{S} \\
& \quad \lesssim(1+|t|)^{-21 / 20}\|F\|_{S^{+}}\|G\|_{S^{+}}\|H\|_{S^{+}} .
\end{aligned}
$$

Indeed, with $\tilde{G}$ denoting either $G_{c}$ or $G_{f}$ (and similarly for $\tilde{H}$ ) and using (3.26) and (3.33), we obtain that

$$
\begin{aligned}
\left\|\frac{\pi}{t} \mathcal{R}\left[F_{f}, \tilde{G}, \tilde{H}\right]\right\|_{S}+\left\|\Pi^{t}\left[F_{f}, \tilde{G}, \tilde{H}\right]\right\|_{S} & \lesssim(1+|t|)^{-1}\|F\|_{S}\|G\|_{S}\|H\|_{S} \\
\left\|\frac{\pi}{t} \mathcal{R}\left[F_{f}, \tilde{G}, \tilde{H}\right]\right\|_{L_{x, y}^{2}}+\left\|\Pi^{t}\left[F_{f}, \tilde{G}, \tilde{H}\right]\right\|_{L_{x, y}^{2}} & \lesssim(1+|t|)^{-1}\left\|F_{f}\right\|_{L^{2}}\|\tilde{G}\|_{S}\|\tilde{H}\|_{S} \\
& \lesssim(1+|t|)^{-5 / 4}\|F\|_{S}\|G\|_{S}\|H\|_{S} .
\end{aligned}
$$

Using (2.11) allows us to bound the contribution of this term to (3.36). The terms involving $G_{f}$ and $H_{f}$ can be treated similarly.

Similarly, using (3.26), we see that

$$
\begin{aligned}
\left\|\frac{\pi}{t} \mathcal{R}\left[F_{f}, \tilde{G}, \tilde{H}\right]\right\|_{S}+\left\|\Pi^{t}\left[F_{f}, \tilde{G}, \tilde{H}\right]\right\|_{S} & \lesssim(1+|t|)^{-1}\left\|F_{f}\right\|_{S}\|G\|_{S}\|H\|_{S} \\
& \lesssim(1+|t|)^{-5 / 4}\|F\|_{S^{+}}\|G\|_{S^{+}}\|H\|_{S^{+}} .
\end{aligned}
$$

This bounds the contribution of terms involving $F_{f}$ to the right-hand side of (3.36). The contribution of terms involving $H_{f}$ or $G_{f}$ follows similarly.

Therefore, to show (3.28) and (3.29), it suffices to show that

$$
\left\|\Pi^{t}\left[F_{c}, G_{c}, H_{c}\right]-\frac{\pi}{t} \mathcal{R}\left[F_{c}, G_{c}, H_{c}\right]\right\|_{Z} \lesssim(1+|t|)^{-15 / 14}\|F\|_{S}\|G\|_{S}\|H\|_{S}
$$

and

$$
\left\|\Pi^{t}\left[F_{c}, G_{c}, H_{c}\right]-\frac{\pi}{t} \mathcal{R}\left[F_{c}, G_{c}, H_{c}\right]\right\|_{S} \lesssim(1+|t|)^{-15 / 14}\|F\|_{S^{+}}\|G\|_{S^{+}}\|H\|_{S^{+}} .
$$

The proof of (3.38) follows from Lemma 3.10 and (2.13). Using once again Lemma 3.10 and (2.13), one directly estimates the $L_{x, y}^{2}$ contribution to the $S$ norm in the left-hand side of (3.39). Using in addition a Leibniz rule, one estimates the $\|x F\|_{L_{x, y}^{2}}$ and the $\left\|\partial_{y}^{N} F\right\|_{L_{x, y}^{2}}$ contributions to the $S$-norm in the lefthand side of (3.39) by a use of Lemma 3.10 and (2.13). Finally, the $\left\|\partial_{x}^{N} F\right\|_{L_{x, y}^{2}}$ contribution to the $S$-norm in the left-hand side of (3.39) can be evaluated as follows. Let us first explain how we evaluate the first derivative. To simplify the notation, let us set

$$
T\left[F_{c}, G_{c}, H_{c}\right]=\Pi^{t}\left[F_{c}, G_{c}, H_{c}\right]-\frac{\pi}{t} \mathcal{R}\left[F_{c}, G_{c}, H_{c}\right] .
$$


Then

$$
\begin{aligned}
\partial_{x} T\left[F_{c}, G_{c}, H_{c}\right]= & T\left[\left(\partial_{x} F\right)_{c}, G_{c}, H_{c}\right]+T\left[F_{c},\left(\partial_{x} G\right)_{c}, H_{c}\right]+T\left[F_{c}, G_{c},\left(\partial_{x} H\right)_{c}\right] \\
& +t^{-1 / 4}\left(T\left[\tilde{F}_{c}, G_{c}, H_{c}\right]+T\left[F_{c}, \tilde{G}_{c}, H_{c}\right]+T\left[F_{c}, G_{c}, \tilde{H}_{c}\right]\right),
\end{aligned}
$$

where $\tilde{F}_{c}=\varphi^{\prime}\left(t^{-1 / 4} x\right) F$, and similarly for $\tilde{G}_{c}$ and $\tilde{H}_{c}$. We are now in a position to apply Lemma 3.10 and (2.13) to estimate the first $x$-derivative contribution to the $S$-norm in the left-hand side of (3.39). The estimates for higher-order derivatives can be performed inductively. This completes the proof of Lemma 3.7.

Finally, we can give the proof of Proposition 3.1.

Proof of Proposition 3.1. For $t \in[T / 4, T]$, we may decompose

$$
\begin{aligned}
\mathcal{N}^{t}= & \sum_{\substack{A, B, C \\
\max (A, B, C) \geqslant T^{1 / 6}}} \mathcal{N}^{t}\left[Q_{A} F(t), Q_{B} G(t), Q_{C} H(t)\right] \\
& +\tilde{\mathcal{N}}^{t}\left[Q_{\leqslant T^{1 / 6}} F(t), Q_{\leqslant T^{1 / 6}} G(t), Q_{\leqslant T^{1 / 6}} H(t)\right] \\
& +\Pi^{t}\left[Q_{\leqslant T^{1 / 6}} F(t), Q_{\leqslant T^{1 / 6}} G(t), Q_{\leqslant T^{1 / 6}} H(t)\right] .
\end{aligned}
$$

The first term above contributes to $\mathcal{E}_{1}$ by Lemma 3.2. The second term contains $\mathcal{E}_{2}$, as it can be written by Lemma 3.3 as $\widetilde{\mathcal{E}}_{1}+\mathcal{E}_{2}$ with $\widetilde{\mathcal{E}}_{1}$ giving an acceptable contribution to $\mathcal{E}_{1}$. The third term can be written as

$$
\begin{aligned}
\Pi^{t} & {\left[Q_{\leqslant T^{1 / 6}} F(t), Q_{\leqslant T^{1 / 6}} G(t), Q_{\leqslant T^{1 / 6}} H(t)\right]=\frac{\pi}{t} \mathcal{R}[F(t), G(t), H(t)] } \\
+ & \left(\Pi^{t}\left[Q_{\leqslant T^{1 / 6}} F(t), Q_{\leqslant T^{1 / 6}} G(t), Q_{\leqslant T^{1 / 6}} H(t)\right]\right. \\
- & \left.\frac{\pi}{t} \mathcal{R}\left[Q_{\leqslant T^{1 / 6}} F(t), Q_{\leqslant T^{1 / 6}} G(t), Q_{\leqslant T^{1 / 6}} H(t)\right]\right) \\
& -\left(\frac{\pi}{t} \mathcal{R}\left[Q_{\geqslant T^{1 / 6}} F(t), G(t), H(t)\right]+\frac{\pi}{t} \mathcal{R}\left[Q_{<T^{1 / 6}} F(t), Q_{\geqslant T^{1 / 6}} G(t), H(t)\right]\right. \\
& \left.+\frac{\pi}{t} \mathcal{R}\left[Q_{<T^{1 / 6}} F(t), Q_{<T^{1 / 6}} G(t), Q_{\geqslant T^{1 / 6}} H(t)\right]\right) .
\end{aligned}
$$

The second term on the right-hand side contributes to $\mathcal{E}_{1}$ as per Lemma 3.7 (applied to functions with additional projection $Q_{\leqslant T}$ which does not increase their norm). Note also that, for the $S^{+}$-norm, we estimate the norm of the difference as the sum of the norms. The third term on the right-hand side also contributes to $\mathcal{E}_{1}$, as seen by invoking Remark 3.8 and using the estimate

$$
\left\|Q_{\geqslant T^{1 / 6}} F\right\|_{S} \lesssim T^{-2 / 3}\|F\|_{S^{+}} .
$$

This finishes the proof of Proposition 3.1. 


\section{The resonant system}

Here, we review some useful facts about the resonant system which will be at the heart of the asymptotic analysis of (1.1). The resonant system is defined for a vector $a=\left\{a_{p}\right\}_{p \in \mathbb{Z}^{d}}$ as (of course, $R$ is very much related to $\mathcal{R}$ defined in (1.3), and properties of $R$ will directly imply similar properties for $\mathcal{R}$ )

$$
i \partial_{t} a_{p}(t)=\sum_{(p, q, r, s) \in \Gamma_{0}} a_{q}(t) \overline{a_{r}(t)} a_{s}(t)=: R[a(t), a(t), a(t)]_{p} .
$$

This is a Hamiltonian system for the symplectic form

$$
\Omega\left(\left\{a_{p}\right\},\left\{b_{q}\right\}\right)=\operatorname{Im}\left[\sum_{p \in \mathbb{Z}^{d}} \overline{a_{p}} b_{p}\right]=\operatorname{Re}\left\langle-i\left\{a_{p}\right\},\left\{b_{p}\right\}\right\rangle_{l_{p}^{2} \times l_{p}^{2}}
$$

and Hamiltonian

$$
\begin{aligned}
\mathcal{H}(a) & :=\langle R(a, a, a), a\rangle_{l_{p}^{2} \times l_{p}^{2}}=\sum_{(p, q, r, s) \in \Gamma_{0}} a_{p} \overline{a_{q}} a_{r} \overline{a_{s}}=\sum_{\lambda \in \mathbb{Z}} \sum_{\mu \in \mathbb{Z}^{d}}\left|\sum_{\substack{p-q=\mu,|p|^{2}-|q|^{2^{2}}=\lambda}} a_{p} \bar{a}_{q}\right|^{2} \\
& =\left\|e^{i s \Delta_{\mathbb{T}^{d}}} \mathcal{F}_{y}^{-1} a\right\|_{L_{y, s}^{4}\left(\mathbb{T}^{d} \times[0,2 \pi]\right)}^{4} .
\end{aligned}
$$

In addition, for any function $g$, we write

$$
\begin{aligned}
\frac{d}{d t} \sum_{p \in \mathbb{Z}^{d}} g(p) a_{p} \overline{a_{p}} & =2 \sum_{p \in \mathbb{Z}^{d}} g(p) \operatorname{Re}\left\{\overline{a_{p}} \partial_{t} a_{p}\right\} \\
& =-i \sum_{(p, q, r, s) \in \Gamma_{0}}\left\{g(p) \overline{a_{p}} a_{q} \overline{a_{r}} a_{s}-g(p) a_{p} \overline{a_{q}} a_{r} \overline{a_{s}}\right\}
\end{aligned}
$$

and using symmetry, this becomes

$$
\frac{d}{d t} \sum_{p \in \mathbb{Z}^{d}} g(p) a_{p} \overline{a_{p}}=-\frac{i}{2} \sum_{\substack{p+r=q+s \\|p|^{2}+|r|^{2}=|q|^{2}+|s|^{2}}}[g(p)+g(r)-g(q)-g(s)] \overline{a_{p}} a_{q} \overline{a_{r}} a_{s} .
$$

Hence, upon taking $g(p) \equiv 1, g(p)=p, g(p)=|p|^{2}$, we see that we have conservation of the mass, momentum, and energy:

$$
\operatorname{mass}(a)=\sum_{p \in \mathbb{Z}^{d}}\left|a_{p}\right|^{2}, \quad \operatorname{mom}(a)=\sum_{p \in \mathbb{Z}^{d}} p\left|a_{p}\right|^{2}, \quad \operatorname{energy}(a)=\sum_{p \in \mathbb{Z}^{d}}|p|^{2}\left|a_{p}\right|^{2} .
$$


Another way to recover the first and last of these formulas is to see that $R[a, a, \cdot]$ is a self-adjoint operator on $l_{p}^{2}$ and that

$$
\langle i R[a, a, a], a\rangle_{h_{p}^{1} \times h_{p}^{1}}=0
$$

for all $a \in h_{p}^{1}$.

A first simple remark is that the resonant system is well defined for initial data in $h_{p}^{1}$.

LEMmA 4.1. Let $1 \leqslant d \leqslant 4$. For any $a(0) \in h_{p}^{1}$, there exists a unique global solution $u \in C^{1}\left(\mathbb{R}: h_{p}^{1}\right)$ of (4.1). In addition, higher regularity is preserved in the sense that, if $a(0) \in h_{p}^{s}$, then the solution belongs to $C^{1}\left(\mathbb{R}: h_{p}^{s}\right)$.

Note that this is the reason for our restriction to $d \leqslant 4$ in Theorems 1.1 and 1.2. When $d \geqslant 5$, the flow map of (4.1) cannot even be $C^{3}$ in $h_{p}^{1}$ in any neighborhood of 0 .

Proof. From Lemma 7.1, we see that the mapping $a \mapsto R[a, a, a]$ is locally Lipschitz in $h_{p}^{1}$, uniformly on bounded subsets. A contraction mapping argument gives local well-posedness in $h_{p}^{s}$ for any $s \geqslant 1$ which is extended to a global statement in $h_{p}^{1}$ by (4.3). The preservation of higher regularity is classical.

REMARK 4.2. Small data do not make a difference: using to the symmetry $\left(a_{n}(t)\right) \rightarrow\left(\lambda a_{n}\left(\lambda^{2} t\right)\right)$ enjoyed by (4.1), we can normalize the initial data to any preassigned size $\delta$ in $h_{p}^{s}$. In addition, by a complex conjugation one can pass from the 'focusing' to the 'defocusing' resonant system.

\subsection{Estimation of solutions to the resonant system.}

LEMmA 4.3. (i) Assume that $G_{0} \in S^{(+)}$(here, $S^{(+)}$denotes either $S$ or $S^{+}$) and that $G$ evolves according to (1.3). Then, it holds that, for $t \geqslant 1$,

$$
\begin{gathered}
\|G(\ln t)\|_{Z}=\left\|G_{0}\right\|_{Z} \\
\|G(\ln t)\|_{S^{(+)}} \lesssim(1+|t|)^{\delta^{\prime}}\left\|G_{0}\right\|_{S^{(+)}} .
\end{gathered}
$$

Besides, we may choose $\delta^{\prime} \lesssim\left\|G_{0}\right\|_{Z}^{2}$.

(ii) In addition, we have the following uniform continuity result: if $A$ and $B$ solve (1.3) and satisfy

$$
\sup _{0 \leqslant t \leqslant T}\left\{\|A(t)\|_{Z}+\|B(t)\|_{Z}\right\} \leqslant \theta
$$


and

$$
\|A(0)-B(0)\|_{S^{(+)}} \leqslant \delta,
$$

then, it holds that, for $0 \leqslant t \leqslant T$,

$$
\|A(t)-B(t)\|_{S^{(+)}} \leqslant \delta e^{C \theta^{2} t} .
$$

Proof of Lemma 4.3. The first equality in (4.5) follows from (4.3). For the second, we simply use (7.2) and (7.1) to show that, for $\sigma \geqslant 0$ and fixed $\xi$,

$$
\begin{aligned}
\|\mathcal{F} \mathcal{R}[G, G, G](\xi)\|_{h_{p}^{\sigma}} & \lesssim\|G\|_{Z}^{2}\|\widehat{G}(\xi)\|_{h_{p}^{\sigma}} \\
\left\|\partial_{\xi} \mathcal{F} \mathcal{R}[G, G, G](\xi)\right\|_{l_{p}^{2}} & \lesssim\|G\|_{Z}^{2}\left\|\partial_{\xi} \widehat{G}(\xi)\right\|_{l_{p}^{2}} .
\end{aligned}
$$

An application of Gronwall inequality yields the statement about the $S$-norm in (4.5). For the $S^{+}$-norm, we use again (7.2) and (7.1) to get

$$
\begin{gathered}
\left\|\partial_{\xi} \mathcal{F} \mathcal{R}[G, G, G](\xi)\right\|_{h_{p}^{\sigma}} \lesssim\|G\|_{Z}^{2}\left\|\partial_{\xi} \widehat{G}(\xi)\right\|_{h_{p}^{\sigma}}+\|G\|_{Z}\left\|\partial_{\xi} \widehat{G}\right\|_{h_{p}^{1}}\|\widehat{G}\|_{h_{p}^{\sigma}}, \\
\left\|\partial_{\xi}^{2} \mathcal{F} \mathcal{R}[G, G, G](\xi)\right\|_{l_{p}^{2}} \lesssim\|G\|_{Z}^{2}\left\|\partial_{\xi}^{2} \widehat{G}(\xi)\right\|_{l_{p}^{2}}+\|G\|_{Z}\left\|\partial_{\xi} \widehat{G}\right\|_{h_{p}^{1}}\left\|\partial_{\xi} \widehat{G}\right\|_{h_{p}^{1}} .
\end{gathered}
$$

Bounding first the case $\sigma=1$ and applying inhomogeneous Gronwall estimates, we obtain the bound on the $S^{+}$-norm in (4.5).

The proof of (4.6) is similar, based on the fact that

$$
\begin{aligned}
\partial_{\tau}\left\{\widehat{A}_{p}(\xi)-\widehat{B}_{p}(\xi)\right\}= & i\left\{\mathcal{R}[\widehat{A}(\xi), \widehat{A}(\xi), \widehat{A}(\xi)]_{p}-\mathcal{R}[\widehat{B}(\xi), \widehat{B}(\xi), \widehat{B}(\xi)]_{p}\right\} \\
= & i \mathcal{R}[\widehat{A}(\xi)-\widehat{B}(\xi), \widehat{A}(\xi), \widehat{A}(\xi)]_{p} \\
& +i \mathcal{R}[\widehat{B}(\xi), \widehat{A}(\xi)-\widehat{B}(\xi), \widehat{A}(\xi)]_{p} \\
& +i \mathcal{R}[\widehat{B}(\xi), \widehat{B}(\xi), \widehat{A}(\xi)-\widehat{B}(\xi)]_{p} .
\end{aligned}
$$

4.2. Special dynamics of the resonant system. In view of Theorems 1.1 and 1.2, it seems interesting to elaborate on some asymptotic dynamics for (4.1). From (4.2) and (4.3), we have $d+3$ conserved scalar quantities, and it is not hard to check that they are in involution. Below, we illustrate some simple dynamics related to Remark 1.5 and Corollary 1.7, and finally recall the theorem from [50] leading to the infinite cascade in Corollary 1.4.

REMARK 4.4. To transfer information from a global solution $a(t)$ of (4.1) to a solution of (1.3), all one needs to do is take an initial data of the form

$$
G_{0}(x, y)=\varepsilon_{0} \check{\varphi}(x) g(y),
$$


where $g_{p}=a_{p}(0)$. The solution $G(t)$ to (1.3) with initial data $G_{0}$ as above is given in Fourier space by

$$
\widehat{G}_{p}(t, \xi)=\varphi(\xi) a_{p}\left(\varphi(\xi)^{2} t\right) .
$$

In particular, if $\varphi=1$ on an open interval $I$, then $\widehat{G}_{p}(t, \xi)=a_{p}(t)$ for all $t \in \mathbb{R}$ and $\xi \in I$.

We start with a simple observation that prevents linear scattering.

LEMMA 4.5. Assume that a solves (4.1) and that

$$
\left\|\partial_{t} a\right\|_{l_{p}^{2}} \rightarrow 0 \text { as } t \rightarrow+\infty
$$

Then $a \equiv 0$.

Proof. This follows from the conservation and coercivity of the mass and Hamiltonian:

$$
\mathcal{H}(a)=\left\langle i \partial_{t} a, a\right\rangle_{l_{p}^{2} \times l_{p}^{2}}, \quad\|a(t)\|_{l^{2}}=\operatorname{mass}(a) ;
$$

hence we see that $\mathcal{H}(a)=0$, and (4.2) now implies that $a \equiv 0$.

4.2.1. The case when $d=1$. This case can be integrated explicitly:

$$
i \partial_{t} a_{p}=2 \sum_{q \in \mathbb{Z}}\left|a_{q}\right|^{2} a_{p}-\left|a_{p}\right|^{2} a_{p}
$$

Thus, we see that

$$
a_{p}(t)=e^{i b_{p} t} a_{p}(0), \quad b_{p}=2 \operatorname{mass}(a)-\left|a_{p}(0)\right|^{2} .
$$

In particular, $\left|a_{p}(t)\right|^{2} \equiv\left|a_{p}(0)\right|^{2}$ remains constant in time, and there can be no cascade.

4.2.2. Solutions supported on a rectangle. The simplest genuinely multidimensional solution is supported on a rectangle $\left(p_{0}, p_{1}, p_{2}, p_{3}\right)$. We refer to $[30,44]$ for related (and more elaborate) computations. Letting

$$
a_{j}=a_{p_{j}}, \quad j \in\{0,1,2,3\}=\mathbb{Z} / 4 \mathbb{Z},
$$

we see that (4.1) becomes

$$
i \partial_{t} a_{j}=2 a_{j+1} \overline{a_{j+2}} a_{j-1}+2\left(\left|a_{j+1}\right|^{2}+\left|a_{j+2}\right|^{2}+\left|a_{j-1}\right|^{2}\right) a_{j}+\left|a_{j}\right|^{2} a_{j} .
$$


An application of Gronwall's inequality shows that a solution initially supported in a rectangle will remain supported on this rectangle. Besides, we can see that mass, Hamiltonian, and momentum in two different directions in the span of the rectangle are generically independent and thus the Liouville-Arnold-Jost theorem provides many 4-torii of solutions.

There is a simple subsystem corresponding to the case when

$$
b_{0}(t):=a_{0}(t)=a_{2}(t), \quad b_{1}(t):=a_{1}(t)=a_{3}(t),
$$

which, by an application of Gronwall's inequality can be seen to be invariant by the flow. Besides, (4.1) becomes

$$
i \partial_{t} b_{j}=-\left|b_{j}\right|^{2} b_{j}+4 b_{j}\left(\left|b_{j}\right|^{2}+\left|b_{j+1}\right|^{2}\right)+2 b_{j+1}^{2} \overline{b_{j}}, \quad j \in\{0,1\}=\mathbb{Z} / 2 \mathbb{Z} .
$$

Without any loss of generality, we can normalize the initial data so that $\left|b_{0}\right|^{2}+$ $\left|b_{1}\right|^{2}=1$ (see Remark 4.2). We now move to polar coordinates, and define

$$
I_{j}=\left|b_{j}\right|^{2} \quad \text { and } \quad \theta_{j}=\arg b_{j}-4 m t, \quad m=\operatorname{mass}(b)=\left|b_{0}\right|^{2}+\left|b_{1}\right|^{2} .
$$

A direct calculation shows that the system satisfied by $\left(I_{j}, \theta_{j}\right)$ is given by

$$
\dot{\theta}_{j}=I_{j}-2 I_{j+1} \cos \left(2\left(\theta_{j+1}-\theta_{j}\right)\right), \quad \dot{I}_{j}=4 I_{j} I_{j+1} \sin \left(2\left(\theta_{j+1}-\theta_{j}\right)\right) .
$$

The conservation of mass and Hamiltonian translates in the above variables into

$$
I_{0}+I_{1}=1 ; \quad \tilde{h}\left(I_{0}, I_{1}, \theta_{0}, \theta_{1}\right)=\frac{1}{2}\left(I_{0}^{2}+I_{1}^{2}\right)-2 I_{0} I_{1} \cos \left(2\left(\theta_{0}-\theta_{1}\right)\right)=c s t .
$$

It is easy to see either by direct verification or by noticing that all the above variable changes are symplectic that the above system (4.9) is Hamiltonian. Let $r=I_{0}$, and define $\varphi=\theta_{1}-\theta_{0}$. The system satisfied by $(r, \varphi)$ is the following:

$$
\dot{\varphi}=(1-2 r)(1+2 \cos 2 \varphi), \quad \dot{r}=4 r(1-r) \sin 2 \varphi,
$$

which is also Hamiltonian, with energy

$$
h(\varphi, r)=r(1-r)[1+2 \cos (2 \varphi)] .
$$

Due to our mass normalization, we have that $r \in[0,1]$ for all time. Notice (also notice that the energy curve $h=0$ supports only two types of orbit, namely given by $\cos 2 \varphi=-\frac{1}{2}$ and $\dot{r}= \pm 2 \sqrt{3} r(1-r)$, which leads to the heteroclinic orbit at the basis of the construction in [30]) that $\left(I_{0}, I_{1}, \theta_{0}, \theta_{1}\right)$ can all be derived from the knowledge of $(\varphi, r)$ and (4.9).

Looking at the phase diagram inside the rectangle defined by the invariant lines $\{r=0\},\{r=1\},\{\varphi=-\pi / 3\}$ and $\{\varphi=\pi / 3\}$, we notice that $(\varphi=0$, $\left.r=\frac{1}{2}\right)$ is the only stationary point, and therefore the level sets $\{h(\varphi, r)=a\}$ 


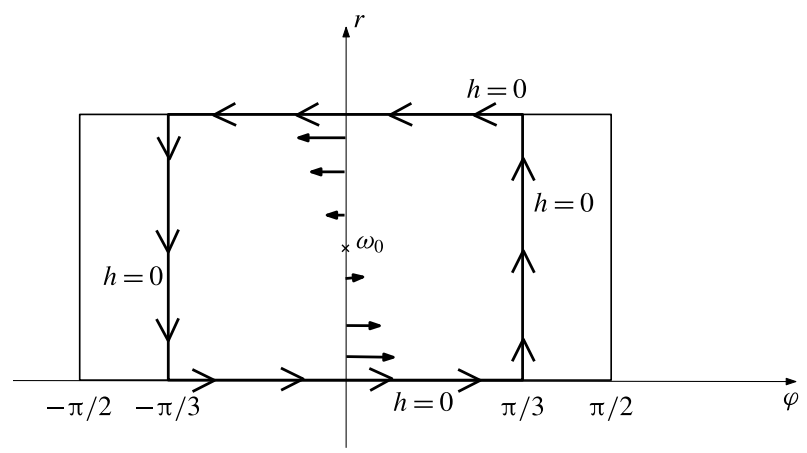

Figure 1. Phase diagram for $h(\varphi, r)$.

foliate this rectangle as $a$ ranges between the two extreme values: 0 attained at the boundary and 3/4 attained at the center (see Figure 1).

An application of the Liouville-Arnold-Jost theorem shows that, if we start with initial data $(\varphi=0, r=1-\delta)$, then the solution to (4.11) will be periodic in time, with energy level given by $h(0,1-\delta)=3 \delta(1-\delta)$. If $2 T$ is the period, then the value of $r(t)$ will oscillate between the two extreme values of $\delta$ and $1-\delta$ attained at respectively even and odd multiples of $T$.

All in all, we have the following.

Proposition 4.6. Let $\Lambda$ be a rectangle with vertices $p_{1}, p_{2}, p_{3}, p_{4}$ in $\mathbb{Z}^{d}$. Let $\Lambda_{1}=\left\{p_{1}, p_{3}\right\}$ and $\Lambda_{2}=\left\{p_{2}, p_{4}\right\}$ denote the diagonally opposite pairs.

(1) There exist solutions to (4.1) supported on $R$ that are quasiperiodic with up to four periods (four angle variables).

(2) For any $\delta>0$, there exists a periodic-in-time solution $\left(a_{n}(t)\right)$ of $(4.1)$ supported on $R$, with period $2 T$, and satisfying the following:

$$
\begin{aligned}
& \operatorname{mass}\left[\left(a_{n}(0)\right), \Lambda_{1}\right]=\delta \quad \text { and } \quad \operatorname{mass}\left[a_{n}(0), \Lambda_{2}\right]=1-\delta, \\
& \operatorname{mass}\left[\left(a_{n}(T)\right), \Lambda_{1}\right]=1-\delta \quad \text { and } \operatorname{mass}\left[a_{n}(T), \Lambda_{2}\right]=\delta,
\end{aligned}
$$

where we denote mass $\left[\left(a_{n}\right), \Lambda_{j}\right]=\sum_{n \in \Lambda_{j}}\left|a_{n}\right|^{2}$, and $T$ is half the period of motion.

REMARK 4.7. While the above solutions were supported on one rectangle in $\mathbb{Z}^{d}$, one can actually construct the same solutions on any (possibly infinite) family of rectangles $\left\{\Lambda_{l}\right\}$ as long as the system (4.1) decouples to each rectangle. This can be achieved by making sure that the rectangles $\Lambda_{l}$ do not form resonant 
interactions between them. We refer to [50] for the precise definitions. In particular, the set $\Lambda_{1}, \Lambda_{2}$, and $\Lambda=\Lambda_{1} \cup \Lambda_{2}$ in Proposition 4.6 can be made infinite.

The solutions constructed in the above proposition directly yield time-periodic and quasiperiodic solutions of (1.3) by setting $\widehat{G}_{p}(t, \xi)=\frac{1}{2} \mathbf{1}_{[-1,1]}(\xi) a_{p}(t)$. However, such solutions are in $H^{N}\left(\mathbb{R} \times \mathbb{T}^{d}\right)$ but not in $S$ or $S^{+}$. To fix this caveat, one can use, instead of $\mathbf{1}_{[-1,1]}(\xi)$, a smooth even function $\psi_{\epsilon}(\xi)$ satisfying

$$
\begin{cases}\psi_{\epsilon}(\xi)=\frac{1}{2} & |\xi| \leqslant 1-\epsilon, \\ \psi_{\epsilon}(\xi)=0 \quad & |\xi| \geqslant 1,\end{cases}
$$

and a smooth nonnegative nonincreasing interpolant on the interval $[1,1+\epsilon]$. One can also arrange things so that $\left\|\psi_{\epsilon}\right\|_{S^{+}} \leqslant 3 \epsilon^{-2}$. If the initial data for $G$ is taken to be $\widehat{G}_{p}(0, \xi)=\epsilon^{3} \psi_{\epsilon}(\xi) a_{p}(0)$, then the obtained solution $G(t)$ is given by $\widehat{G}_{p}(t, \xi)=\epsilon^{3} \psi_{\epsilon}(\xi) a_{p}\left(\epsilon^{3} \psi_{\epsilon}(\xi)^{2} t\right)$ (see Remark 4.2). Notice that the $S$-norm and the $S^{+}$-norm of $G(0)$ are then $O(\epsilon)$.

4.2.3. Infinite cascade. An important result for us is the existence of infinitely growing solutions to (4.1), as proved in [50, Theorem 1.6 with $R=0$ ]. We give a self-contained constructive proof of this result that follows from simple adaptations of the more recent work [45] in order to obtain an explicit global solution with a lower bound on the growth rate of its Sobolev norms. Our main result here is the following.

Proposition 4.8. Let $d \geqslant 2$ and $s>1$. There exist global solutions to (4.1) in $C\left(\mathbb{R}: h_{p}^{s}\right)$ such that

$$
\sup _{t>0}\|a(t)\|_{h_{p}^{s}}=\infty .
$$

More precisely, for any $\varepsilon>0$, there exists a solution $a(t) \in C\left(\mathbb{R}: h_{p}^{s}\right)$ such that for some sequence of times $t_{k} \rightarrow \infty$ we have that

$$
\|a(0)\|_{h_{p}^{s}} \leqslant \varepsilon, \quad\left\|a\left(t_{k}\right)\right\|_{h_{p}^{s}} \gtrsim\left(\log t_{k}\right)^{C}
$$

for some $C>0$.

By Remark 4.4, this yields a global solution of (1.3) in $C\left(\mathbb{R}: H^{s}\left(\mathbb{R} \times \mathbb{T}^{d}\right)\right)$ whose $H^{s}$ norm grows at the rate (4.13). For the NLS equation (1.1) (by Theorem 1.2), this yields a growth of $\left(\log \log t_{k}\right)^{C}$. We have no reason to believe that the rate of growth in (4.13), or the implied rate for (1.1), is optimal. In addition, it is tempting to believe that, for any $s>1$, there exists a solution in $H^{\infty}\left(\mathbb{R} \times \mathbb{T}^{2}\right)$ whose $H^{s}$ norm blows up in infinite time. 
We now move to the proof of Proposition 4.8. We start by noticing that it is enough to prove the result on $\mathbb{Z}^{2}$, as this gives a solution of (4.1) on $\mathbb{Z}^{d}$ satisfying the same properties. In addition, we note as in [30] that, by an easy change of unknown,

$$
a_{p}(t) \rightarrow a_{p}(t) e^{i G t}, \quad G=2\left\|a_{p}\right\|_{l_{p}^{2}}^{2},
$$

we may reduce (4.1) to the system

$$
i \partial_{t} a_{p}=-\left|a_{p}\right|^{2} a_{p}+\sum_{(p, q, r, s) \in \Gamma_{0}^{\prime}} a_{q} \bar{a}_{r} a_{s},
$$

where $\Gamma_{0}^{\prime}$ corresponds to the nondegenerate rectangles $(p, q, r, s)$, that is, rectangles with positive area. Of course, the transformation (4.14) does not change the $h_{p}^{s}$-norms, and it may be easily inverted.

Next, we recall the following result, which is essentially contained in [45, Theorem 3-bis and Appendix C].

TheOREM $4.9[30,45,46]$. Fix $\gamma \gg 1$. There exist $C, v, K>0$ (independent of $\gamma$ ) such that. for any $N$ sufficiently large, there exist a finite set $S_{N} \subset \mathbb{Z}^{2}$ and a solution $a^{(N)}(t)=\left(a_{k}^{(N)}(t)\right)_{k \in \mathbb{Z}^{2}}$ of (4.15) such that

- $\left(0_{\Lambda}\right)$ if $\left(p_{0}, q_{0}, r_{0}\right)$ form a right-angled triangle $\left(\right.$ at $\left.q_{0}\right)$ in $S_{N}$, then $r_{0}+p_{0}-q_{0} \in$ $S_{N}$, that is, a rectangle has either four or (strictly) fewer than three of its vertices inside $S_{N}$;

- $\left(I_{\Lambda}\right) S_{N}=\Lambda_{1} \cup \Lambda_{2} \cup \cdots \cup \Lambda_{N} \subset B\left(0,\left[\left(K^{N}\right) !\right]^{N}\right)$;

- $\left(I I_{\Lambda}\right) \Lambda_{j}$ contains $2^{N-1}$ points, $1 \leqslant j \leqslant N$;

- $\left(I I I_{\Lambda}\right)$ if $\Lambda_{j} \subset B(0, r)$, then $\Lambda_{j+1} \subset B(0, \sqrt{2} r)$;

- $\left(I V_{\Lambda}\right)$ there exists $R>0$ such that $\Lambda_{1}$ is contained in a disc of radius $R \leqslant$ $\left[\left(K^{N}\right) !\right]^{N}$ and $\Lambda_{N-1}$ contains at least two points at distance $R 2^{(N-10) / 2}$ from the origin;

and the solution $a^{(N)}(t)=\left(a_{k}^{(N)}(t)\right)_{k \in \mathbb{Z}^{2}}$ satisfies

- $\left(I_{a}\right)$ for all times, $a^{(N)}(t)$ is supported on $S_{N}$, and, for any $j=1, \ldots, N$, $a^{(N)}(t)$ is constant on $\Lambda_{j}$, that is, $a_{k}^{(N)}(t)=b_{j}^{(N)}(t)$ for $k \in \Lambda_{j}$;

- $\left(I I_{a}\right) a^{(N)}(t)$ cascades energy in the sense that there exists $T_{N}$ such that

$$
\begin{gathered}
1 \geqslant\left|b_{3}^{(N)}(0)\right|>1-\delta^{\nu}, \quad\left|b_{N-1}^{(N)}\left(T_{N}\right)\right|>1-\delta^{\nu}, \\
\left|b_{j}^{(N)}(0)\right|<\delta^{\nu} \quad \text { for } j \neq 3 \quad\left|b_{j}^{(N)}\left(T_{N}\right)\right|<\delta^{\nu} \quad \text { for } j \neq N-1,
\end{gathered}
$$

where $\delta=e^{-\gamma N}$; 
- $\left(\right.$ I I I $\left.I_{a}\right)$ it holds that $0<T_{N}<C \gamma N^{2}$.

REMARK 4.10. The growth in Proposition 4.8 can be specified to be

$$
\left\|a\left(t_{k}\right)\right\|_{h_{p}^{s}} \gtrsim \exp \left(c F^{-1}\left(t_{k}^{c}\right)\right)
$$

where $F(N)$ is an increasing function lower bounding the radius of a ball containing the first generation appearing in $I V_{\Lambda}$ above (here, $F^{-1}$ denotes the inverse function of $F$ ) and $c>0$ is a small constant.

Proof of Proposition 4.8. The needed solution is constructed using the observation (see [50]) that compactly supported solutions of (4.15) of disjoint support can be easily superposed by appropriately positioning them in the lattice $\mathbb{Z}^{2}$.

Fix $s>1$ and $\gamma>2 s / \nu$. We start by applying Theorem 4.9 for every $N=$ $j \in \mathbb{N}, j \geqslant N_{0}(\gamma)$. This gives a family of sets $S_{j}=\Lambda_{1}^{j} \cup \Lambda_{2}^{j} \cup \cdots \cup \Lambda_{j}^{j}$ satisfying $\left(I_{\Lambda}\right)-\left(I V_{\Lambda}\right)$ and solutions $a^{(j)}(t)$ of (4.15) satisfying $\left(I_{a}\right)-\left(I I I_{a}\right)$.

In addition, considering $\left(I I I_{\Lambda}-I V_{\Lambda}\right)$, we see that we may assume that there exists $\frac{1}{2}\left[\left(K^{N}\right) !\right]^{N} \leqslant R_{j} \leqslant\left[\left(K^{N}\right) !\right]^{N}$ such that

$$
\Lambda_{p}^{j} \subset B\left(0, \sqrt{2}^{p} R_{j}\right) \quad \text { for } 1 \leqslant p \leqslant j ; \quad \Lambda_{j-1}^{j} \cap B\left(0,2^{(j-20) / 2} R_{j}\right)^{c} \neq \emptyset .
$$

Next, we claim that we can construct by induction a sequence of vectors $\left\{v_{j}\right\}_{j \geqslant N_{0}} \subset \mathbb{Z}^{2}$ such that

$$
v_{N_{0}}=0, \quad\left|v_{j}\right| \leqslant 2^{10 j}
$$

and for any nondegenerate rectangle $\left(p_{0}, q_{0}, r_{0}, s_{0}\right)$ with three vertices included in

$$
\Xi=\bigcup_{j \geqslant N_{0}}\left(v_{j}+S_{j}\right) .
$$

Then $\left\{p_{0}, q_{0}, r_{0}, s_{0}\right\} \subset \Xi$, and we have the following property:

$$
\begin{gathered}
\text { if }\left\{p_{0}, q_{0}, r_{0}, s_{0}\right\} \cap\left(v_{j}+S_{j}\right) \neq \emptyset \quad \text { and } \\
\left\{p_{0}, q_{0}, r_{0}, s_{0}\right\} \cap\left(v_{k}+S_{k}\right) \neq \varnothing \quad \text { then } j=k .
\end{gathered}
$$

The existence of this sequence of vectors is proved inductively using Lemma 4.11 below (at the $n$th step, take $\Xi_{n}=\bigcup_{1 \leqslant j \leqslant n-1}\left(S_{j}+v_{j}\right)$ which has $O\left(n 2^{n}\right)$ elements). We then easily see that any nondegenerate right-angled triangle in $\Xi$ must belong to exactly one $v_{j}+S_{j}$. The fact that the fourth corner of a rectangle necessarily belongs to $\Xi$ follows from the fact that each 
component $v_{j}+S_{j}$ satisfies this property thanks to $\left(0_{\Lambda}\right)$ above. Choosing any such sequence $\left\{v_{j}\right\}_{j \geqslant N_{0}}$, we define the following sequence of initial data $A^{(p)}(0)$ for (4.1) to be given by

$$
A^{(p)}(0)=\sum_{N_{0} \leqslant j \leqslant p} \lambda_{j} a_{k-v_{j}}^{(j)}(0),
$$

where $\lambda_{j}=\left(\varepsilon / j^{10}\right) 2^{-j / 2} R_{j}^{-s}$ is a normalization factor. Note that, for any $v \in \mathbb{Z}^{2}$, $a_{k-v}^{(j)}(t)$ is also a solution of (4.15). Using $\left(I_{a}-I I_{a}\right)$, (4.16), and (4.17), we therefore see that

$$
\left\|A^{(p+1)}(0)-A^{(p)}(0)\right\|_{h_{p}^{s}}^{2} \lesssim p^{-20} \varepsilon^{2},
$$

so that $A^{(p)}(0)$ is a Cauchy sequence of initial data in $h_{p}^{s}$, and therefore it converges to some $A(0) \in h_{p}^{s}\left(\mathbb{Z}^{2}\right)$. Moreover, $A(0)$ satisfies the first property in (4.13).

What remains to show is that the solution $A(t)$ of (4.1) with initial data $A(0)$ satisfies the second property in (4.13). We start by noticing that, by (4.18) (recall that $\left(p_{0}, q_{0}, r_{0}, s_{0}\right) \in \Gamma_{0}^{\prime}$ if and only if $\left(p_{0}, q_{0}, r_{0}, s_{0}\right)$ are the vertices of a nondegenerate rectangle), the solution $A^{p}(t)$ with initial data $A^{p}(0)$ is given by

$$
A^{p}(t)=\sum_{N_{0} \leqslant j \leqslant p} \lambda_{j} a_{k-v_{j}}^{(j)}\left(\lambda_{j}^{2} t\right)
$$

As a result, we see that, if $m \geqslant n$ and $k \in v_{n}+S_{n}$, then

$$
A_{k}^{(m)}(t)=A_{k}^{(n)}(t)=\lambda_{n} a_{k-v_{n}}^{(n)}\left(\lambda_{n}^{2} t\right)=\lambda_{n} \sum_{1 \leqslant \ell \leqslant n} b_{\ell}^{(n)}\left(\lambda_{n}^{2} t\right) \mathbf{1}_{\Lambda_{\ell}^{n}}\left(k-v_{n}\right) .
$$

By continuity of the flow, this also holds for $A^{(m)}(t)$ replaced by $A(t)$. In particular, using $\left(I V_{\Lambda}, I_{a}-I I_{a}\right)$ and (4.17), we see that

$$
\left\|A\left(\lambda_{n}^{-2} T_{n}\right)\right\|_{h^{s}}^{2} \geqslant \lambda_{n}^{2} \sum_{k \in \Lambda_{n-1}^{n}}\left|b_{n-1}^{(n)}\left(T_{n}\right)\right|^{2} \cdot\left|k+v_{n}\right|^{2 s} \gtrsim n^{-20} \varepsilon^{2} 2^{n(s-1)} .
$$

Setting $s_{n}=\lambda_{n}^{-2} T_{n}$, and noticing that $s_{n} \lesssim\left[\left((10 K)^{n}\right) !\right]^{n}$, which implies that $\log \log s_{n} \lesssim n$, finishes the proof.

We now present the lemma justifying the existence of the sequence $\left\{v_{j}\right\}$ above.

LEMmA 4.11. Let $\Xi \subset \mathbb{Z}^{2}$ have cardinality $O\left(j 2^{j}\right)$, and let $S_{j}$ be the set obtained from Theorem 4.9 with $N=j$. Then there exists $v \in \mathbb{Z}^{2}$ with $|v| \leqslant 2^{10 j}$ 
such that for any nondegenerate right-angled triangle $\left(p_{0}, q_{0}, r_{0}\right)$ we have the following property:

$$
\begin{aligned}
& \text { if }\left|\left\{p_{0}, q_{0}, r_{0}\right\} \cap \Xi\right| \geqslant 2, \quad \text { then }\left\{p_{0}, q_{0}, r_{0}\right\} \cap\left(v+S_{j}\right)=\emptyset, \\
& \text { if }\left|\left\{p_{0}, q_{0}, r_{0}\right\} \cap\left(v+S_{j}\right)\right| \geqslant 2, \quad \text { then }\left\{p_{0}, q_{0}, r_{0}\right\} \cap \Xi=\emptyset .
\end{aligned}
$$

Proof. Let $\mathcal{L}$ denote the set of directions of lines joining two points of $\Xi$ or two points of $S_{j}$, or directions which are orthogonal to such lines. $\mathcal{L}$ has cardinality at most $2^{3 j}$, and there exists a vector $v^{\prime}$ of length at most $2^{4 j}$ which is not contained in $\mathcal{L}$.

We now define

$$
\mathcal{A}=\left\{(p, q, r), p, q \in \Xi, r \in S_{j}\right\}, \quad \mathcal{B}=\left\{(p, q, r), p \in \Xi, q, r \in S_{j}\right\} .
$$

We claim that, for any $(p, q, r) \in \mathcal{A}$, the condition ' $\left(C_{p q r}^{1}\right):\left(p, q, r+\lambda v^{\prime}\right)$ form a right-angled triangle' has at most two solutions $\lambda \in \mathbb{R}$ and that similarly, for any $(p, q, r) \in \mathcal{B}$, the condition ' $\left(C_{p, q, r}^{2}\right):\left(p, q+\lambda v^{\prime}, r+\lambda v^{\prime}\right)$ form a rightangled triangle' has at most two solutions.

By translation invariance, it suffices to prove the first claim. If the right angle is at $p$ or $q$, then the proof is direct, since $v^{\prime}$ is neither orthogonal nor parallel to $p-q$. If the right angle happens at $r+\lambda v^{\prime}$, then $r+\lambda v^{\prime}$ belongs to the circle of diameter $(p, q)$, and a line directed by $v^{\prime}$ will intersect this circle in at most two points.

We now observe that $|\mathcal{A}|+|\mathcal{B}| \lesssim 2^{4 j}$, and therefore we may choose $\lambda \in \mathbb{Z} \cap$ $\left[0,2^{5 j}\right]$ such that $\left(C_{p q r}^{1}\right)$ and $\left(C_{p q r}^{2}\right)$ are never satisfied. We now set $v=\lambda v^{\prime}$.

\section{Modified wave operators}

We start the proof of our main results with the slightly easier task of constructing (modified) wave operators for (1.1). The following implies Theorem 1.2.

THEOREM 5.1. There exists $\varepsilon>0$ such that, if $U_{0} \in S^{+}$satisfies

$$
\left\|U_{0}\right\|_{S^{+}} \leqslant \varepsilon
$$

and if $\widetilde{G}$ is the solution of (1.3) with initial data $U_{0}$, then there exists $U$, a solution of (1.1), such that $e^{-i t \Delta_{\mathbb{R} \times \mathbb{T}^{d}}} U(t) \in C((0, \infty): S)$ and

$$
\left\|e^{-i t \Delta_{\mathbb{R} \times \mathbb{T}^{d}}} U(t)-\widetilde{G}(\pi \ln t)\right\|_{S} \rightarrow 0 \quad \text { as } t \rightarrow+\infty .
$$


Proof. This follows by a fixed point argument. We let $G(t)=\widetilde{G}(\pi \ln t)$, and define the mapping

$$
\begin{aligned}
& \Phi(F)(t) \\
& =-i \int_{t}^{\infty}\left\{\mathcal{N}^{\sigma}[F+G, F+G, F+G]-\frac{\pi}{\sigma} \mathcal{R}[G(\sigma), G(\sigma), G(\sigma)]\right\} d \sigma
\end{aligned}
$$

and the space (of course continuing a solution $U$ of $(1.1)$ on the interval $(0,1)$ is direct)

$$
\begin{aligned}
\mathfrak{A} & :=\left\{F \in C^{1}((1, \infty): S):\|F\|_{\mathfrak{A}} \leqslant \varepsilon_{1}\right\} \\
\|F\|_{\mathfrak{A}} & :=\sup _{t>1}\left\{(1+|t|)^{\delta}\|F(t)\|_{S}+(1+|t|)^{2 \delta}\|F(t)\|_{Z}+(1+|t|)^{1-\delta}\left\|\partial_{t} F(t)\right\|_{S}\right\},
\end{aligned}
$$

and we claim that, if $\varepsilon$ is sufficiently small, there exists $\varepsilon_{1}$ such that $\Phi$ defines a contraction on the complete metric space $\mathfrak{A}$ endowed with the metric $\|\cdot\|_{\mathfrak{A}}$.

We now decompose

$$
\begin{aligned}
\mathcal{N}^{t} & {[F+G, F+G, F+G]-\frac{\pi}{t} \mathcal{R}[G, G, G] } \\
& =\mathcal{E}^{t}[G, G, G]+\mathcal{L}^{t}[F, G]+\mathcal{Q}^{t}[F, G],
\end{aligned}
$$

where $\mathcal{E}^{t}[G, G, G]$ is defined as in (3.1) and

$$
\begin{aligned}
\mathcal{L}^{t}[F, G] & :=2 \mathcal{N}^{t}[G, G, F]+\mathcal{N}^{t}[G, F, G], \\
\mathcal{Q}^{t}[F, G] & :=2 \mathcal{N}^{t}[F, F, G]+\mathcal{N}^{t}[F, G, F]+\mathcal{N}^{t}[F, F, F] .
\end{aligned}
$$

We will show that, whenever $F, F_{1}, F_{2} \in \mathfrak{A}$,

$$
\begin{aligned}
& \left\|\int_{t}^{\infty} \mathcal{E}^{\sigma}[G, G, G] d \sigma\right\|_{\mathfrak{A}} \lesssim \varepsilon^{3}, \\
& \left\|\int_{t}^{\infty} \mathcal{L}^{\sigma}[F, G] d \sigma\right\|_{\mathfrak{A}} \lesssim \varepsilon^{2}\|F\|_{\mathfrak{A}}, \\
& \left\|\int_{t}^{\infty} \mathcal{Q}^{\sigma}[F, G] d \sigma\right\|_{\mathfrak{A}} \lesssim \varepsilon\|F\|_{\mathfrak{A}}^{2}, \\
& \left\|\int_{t}^{\infty}\left\{\mathcal{Q}^{\sigma}\left[F_{1}, G\right]-\mathcal{Q}^{\sigma}\left[F_{2}, G\right]\right\} d \sigma\right\|_{\mathfrak{A}} \lesssim \varepsilon \varepsilon_{1}\left\|F_{1}-F_{2}\right\|_{\mathfrak{A}} .
\end{aligned}
$$

Once (5.3) is shown, the proof is complete.

Recall that, if $\varepsilon \lesssim \delta^{1 / 2}$ and $F \in \mathfrak{A}$ (see Lemma 4.3 for the estimates on $G$ ),

$$
\begin{gathered}
(1+|t|)^{2 \delta}\|F(t)\|_{Z}+(1+|t|)^{\delta}\|F(t)\|_{S}+(1+|t|)^{1-\delta}\left\|\partial_{t} F(t)\right\|_{S} \lesssim \varepsilon_{1}, \\
\|G(t)\|_{S^{+}}+(1+|t|)\left\|\partial_{t} G(t)\right\|_{S^{+}} \lesssim \varepsilon(1+|t|)^{\delta / 100} \\
\|G(t)\|_{Z} \lesssim \varepsilon .
\end{gathered}
$$

Using (2.14), the two last inequalities of (5.3) follow. 
We now turn to the first inequality in (5.3). Using (2.14) again (see also (4.7)), we easily see that

$$
\left\|\mathcal{E}^{t}[G, G, G]\right\|_{S} \leqslant\left\|\mathcal{N}^{t}[G, G, G]\right\|_{S}+\frac{1}{t}\|\mathcal{R}[G, G, G]\|_{S} \lesssim(1+|t|)^{-1+\delta} \varepsilon^{3} .
$$

This controls the time derivative in the $\mathfrak{A}$-norm. Independently, using (5.4) with (3.5) in Proposition 3.1, we obtain that

$$
\begin{aligned}
& \left\|\int_{t}^{\infty} \mathcal{E}^{\sigma}(G, G, G) d \sigma\right\|_{S} \lesssim \varepsilon^{3}(1+|t|)^{-\delta}, \\
& \left\|\int_{t}^{\infty} \mathcal{E}^{\sigma}(G, G, G) d \sigma\right\|_{Z} \lesssim \varepsilon^{3}(1+|t|)^{-2 \delta} .
\end{aligned}
$$

This gives the first inequality in (5.3).

Now we turn to the second inequality in (5.3). First, using (2.14) and (5.4), we see that

$$
\left\|\mathcal{N}^{t}[G, G, F]\right\|_{S}+\left\|\mathcal{N}^{t}[G, F, G]\right\|_{S} \lesssim \varepsilon^{2} \varepsilon_{1}(1+|t|)^{-1+\delta},
$$

which is sufficient for the time-derivative component of the $\mathfrak{A}$-norm. Using (5.4) with Lemma 3.2 and Lemma 3.3, it only remains to show that

$$
\begin{aligned}
& \|\mathcal{R}[G, G, F]\|_{Z}+\|\mathcal{R}[G, F, G]\|_{Z} \lesssim(1+|t|)^{-2 \delta} \varepsilon^{2} \varepsilon_{1} \\
& \left\|\Pi^{t}[G, G, F]-\frac{\pi}{t} \mathcal{R}[G, G, F]\right\|_{Z}+\left\|\Pi^{t}[G, F, G]-\frac{\pi}{t} \mathcal{R}[G, F, G]\right\|_{Z} \\
& \quad \lesssim(1+|t|)^{-1-2 \delta} \varepsilon^{2} \varepsilon_{1}, \\
& \left\|\Pi^{t}[G, G, F]\right\|_{S}+\left\|\Pi^{t}[G, F, G]\right\|_{S} \lesssim(1+|t|)^{-1-\delta} \varepsilon^{2} \varepsilon_{1} .
\end{aligned}
$$

Using Lemma 7.1, we see that, for any $A, B, C \in Z$,

$$
\|\mathcal{R}[A, B, C]\|_{Z} \lesssim\|A\|_{Z}\|B\|_{Z}\|C\|_{Z}
$$

and the first estimate follows from (5.4). The second estimate follows directly from (3.28). For the third estimate, we use (3.26) to get

$$
\begin{aligned}
& (1+|t|)\left\{\left\|\Pi^{t}[G, G, F]\right\|_{S}+\left\|\Pi^{t}[G, F, G]\right\|_{S}\right\} \\
& \quad \lesssim\|G\|_{\tilde{Z}_{t}}^{2}\|F\|_{S}+\|G\|_{\tilde{Z}_{t}}\|F\|_{\tilde{Z}_{t}}\|G\|_{S} \lesssim \varepsilon^{2} \varepsilon_{1}(1+|t|)^{-\delta} .
\end{aligned}
$$

The proof is complete. 
REMARK 5.2. Observe that a key point in the proof of the existence of a modified wave operator is the fact that the term

$$
\int_{t}^{\infty} \mathcal{E}^{\sigma}[G, G, G] d \sigma
$$

behaves better in the $Z$-norm than $G$ itself. This allows us to get decay in the $S$-norm by assuming the stronger (however, and this is a key point, this norm is only stronger in $x$ and controls the same amount of periodic derivatives as $S$ ) $S^{+}$ control on the solution of (1.3). We also observe that, in the proof of the existence of the modified wave operator, the argument is completely perturbative. We shall see in the next section that, in the proof of modified scattering, the argument is not completely perturbative and relies on the conservation laws of the resonant system.

\section{Small-data scattering}

The goal of this section is to prove a more precise version of Theorem 1.1, which is the main result of this paper.

THEOREM 6.1. There exists $\varepsilon>0$ such that, if $U_{0} \in S^{+}$satisfies

$$
\left\|U_{0}\right\|_{S^{+}} \leqslant \varepsilon
$$

and if $U$ is the solution of (1.1) with initial data $U_{0}$, then $U$ exhibits modified scattering to the resonant dynamics given by (1.3) in the following sense: there exists $G_{0} \in S$ such that, letting $\widetilde{G}$ be the solution of $(1.3)$ with initial data $\widetilde{G}(0)=$ $G_{0}$, it holds that

$$
\|F(t)-\widetilde{G}(\pi \ln t)\|_{S} \rightarrow 0 \quad \text { as } t \rightarrow+\infty,
$$

where $F(t)=e^{-i t \Delta_{\mathbb{R} \times \mathbb{T}^{d}}} U(t)$.

6.1. Global bounds. Before we turn to the asymptotic behavior of solutions, we need to obtain good global bounds. This is the purpose of the following.

Proposition 6.2. There exists $\varepsilon>0$ such that any initial data $u_{0} \in S^{+}$ satisfying (6.1) generates a global solution of (1.1). Moreover, for any $T>0$, it holds that

$$
\|F(t)\|_{X_{T}^{+}} \leqslant 2 \varepsilon
$$

where $F$ is defined in Theorem 6.1. 
In the case when $d \leqslant 3$, global existence can be established in a much more general setting (namely, $U_{0} \in H^{1}\left(\mathbb{R} \times \mathbb{T}^{d}\right.$ ) is sufficient; see [59]). However, for $d=4$, due to the supercritical nature of the nonlinearity, even global existence seems to require the decay analysis we perform here. In what follows, we emphasize that estimate (6.3) relies on the key nonperturbative identity (4.4).

Proof. Let $F(t)$ be as in the statement of the theorem. Local existence theory and the fact that $t \mapsto\|F(t)\|_{S^{+}}$is $C^{1}$ are classical (see (2.14)); therefore it suffices to show the a priori estimate

$$
\|F\|_{X_{T}^{+}} \leqslant\left\|U_{0}\right\|_{S^{+}}+C\|F\|_{X_{T}^{+}}^{3}
$$

for all $T>0$ and all $U$ solving (1.1) such that $\|F\|_{X_{T}^{+}} \leqslant \sqrt{\varepsilon}$.

We pick $0 \leqslant t \leqslant T$. Clearly, when $0 \leqslant t \leqslant 1$, by (2.14),

$$
\|F(t)-F(0)\|_{S^{+}} \lesssim \sup _{[0, t]}\left\|\partial_{t} F\right\|_{S^{+}} \lesssim\|F\|_{X_{T}^{+}}^{3}
$$

Thus, in the following, we may replace $t=0$ by $t=1$.

We start by noting that, thanks to $(2.14)$, we have that

$$
\begin{gathered}
\left\|\partial_{t} F\right\|_{S}=\left\|\mathcal{N}^{t}[F, F, F]\right\|_{S} \lesssim(1+|t|)^{-1}\|F(t)\|_{S}^{3} \\
\left\|\partial_{t} F\right\|_{S^{+}}=\left\|\mathcal{N}^{t}[F, F, F]\right\|_{S^{+}} \lesssim(1+|t|)^{-1}\|F(t)\|_{S}^{2}\|F(t)\|_{S^{+}},
\end{gathered}
$$

which gives the needed bound for $\partial_{t} F$.

Recall the decomposition in Proposition 3.1. For each fixed $\xi$, multiplying by $\left[1+|p|^{2}\right]$ and taking the inner product with $\widehat{F}(\xi)$, we obtain, after using (4.4), that (a key cancellation appears here in that the resonant term $\mathcal{R}$ disappears, leaving only terms that decay faster)

$$
\frac{d}{d s} \frac{1}{2}\left\|\widehat{F}_{p}(\xi, s)\right\|_{h_{p}^{1}}^{2}=\left\langle\widehat{\mathcal{E}}_{1}(\xi, p, s), \widehat{F}_{p}(\xi, s)\right\rangle_{h_{p}^{1} \times h_{p}^{1}}+\left\langle\partial_{s} \widehat{\mathcal{E}}_{3}(\xi, p, s), \widehat{F}_{p}(\xi, s)\right\rangle_{h_{p}^{1} \times h_{p}^{1}} .
$$

Using (3.3), we have that, for any $\xi$,

$$
\begin{array}{r}
{\left[1+|\xi|^{2}\right] \cdot\left|\int_{0}^{t}\left\langle\widehat{\mathcal{E}}_{1}(\xi, p, s), \widehat{F}_{p}(\xi, s)\right\rangle_{h_{p}^{1} \times h_{p}^{1}} d s\right|} \\
\lesssim\|F\|_{X_{T}^{+}}^{3} \int_{0}^{t}(1+|s|)^{-1-\delta} d s \cdot \sup _{[0, t]}\|F(s)\|_{Z}
\end{array}
$$


and, using (2.14) and (3.3),

$$
\begin{aligned}
{[1+} & \left.|\xi|^{2}\right]\left|\int_{0}^{t}\left\langle\partial_{t} \widehat{\mathcal{E}}_{3}(\xi, p, s), \widehat{F}_{p}(\xi, s)\right\rangle_{h_{p}^{1} \times h_{p}^{1}} d s\right| \\
\leqslant & {\left[1+|\xi|^{2}\right]\left|\left\langle\widehat{\mathcal{E}}_{3}(\xi, p, t), \widehat{F}_{p}(\xi, t)\right\rangle_{h_{p}^{1} \times h_{p}^{1}}\right| } \\
& +\left[1+|\xi|^{2}\right]\left|\left\langle\widehat{\mathcal{E}}_{3}(\xi, p, 0), \widehat{F}_{p}(\xi, 0)\right\rangle_{h_{p}^{1} \times h_{p}^{1}}\right| \\
& +\left[1+|\xi|^{2}\right]\left|\int_{0}^{t}\left\langle\widehat{\mathcal{E}}_{3}(\xi, p, s), \partial_{t} \widehat{F}_{p}(\xi, s)\right\rangle_{h_{p}^{1} \times h_{p}^{1}}\right| \\
& \lesssim\|F\|_{X_{T}^{+}}^{3} \cdot \sup _{t \in[0, T]}\|F(t)\|_{Z}+\|F\|_{X_{T}^{+}}^{6} .
\end{aligned}
$$

Combining the above estimates and integrating in time, we arrive at

$$
\|F(t)\|_{Z} \leqslant\|F(0)\|_{Z}+C\|F\|_{X_{T}^{+}}^{3} .
$$

Independently, using Remark 3.8 and Proposition 3.1, we also see that, so long as $1 \leqslant t \leqslant T$,

$$
\begin{aligned}
\|F(t)-F(1)\|_{S} \lesssim & \left\|\int_{1}^{t} \mathcal{R}[F(s), F(s), F(s)] \frac{d s}{s}\right\|_{S} \\
& +\left\|\int_{1}^{t}\left[\mathcal{E}_{1}(s)+\mathcal{E}_{2}(s)\right] d s\right\|_{S}, \\
\lesssim & (1+|t|)^{\delta}\|F\|_{X_{T}^{+}}^{3},
\end{aligned}
$$

and we may proceed similarly to control the $S^{+}$-norm. This gives the a priori estimate and finishes the proof.

6.2. Asymptotic behavior. We can now give the proof of the main theorem.

Proof of Theorem 6.1. Define $T_{n}=e^{n / \pi}$ and $G_{n}(t)=\widetilde{G}_{n}(\pi \ln t)$, where $\widetilde{G}_{n}$ solves (1.3) with Cauchy data such that $\widetilde{G}_{n}(n)=G_{n}\left(T_{n}\right)=F\left(T_{n}\right)$. We claim that, for all $t \geqslant T_{n}$,

$$
\begin{aligned}
& \left\|G_{n}(t)\right\|_{Z}+(1+|t|)^{-\delta}\left\|G_{n}(t)\right\|_{S}+(1+|t|)^{-5 \delta}\left\|G_{n}(t)\right\|_{S^{+}} \\
& \quad+(1+|t|)^{1-\delta}\left\|\partial_{t} G_{n}(t)\right\|_{S} \lesssim \varepsilon
\end{aligned}
$$

uniformly in $n \geqslant 0$. Indeed, first, using (4.4) and (6.3), we get that

$$
\left\|G_{n}(t)\right\|_{Z}=\left\|\widetilde{G}_{n}(\pi \ln t)\right\|_{Z}=\left\|\widetilde{G}_{n}(n)\right\|_{Z}=\left\|F\left(T_{n}\right)\right\|_{Z} \lesssim \varepsilon
$$


uniformly in $n$. In addition, using also Lemmas 7.1 and 7.4, we see that, uniformly in $n$,

$$
\left\|\partial_{t} G_{n}(s)\right\|_{S} \lesssim s^{-1}\left\|G_{n}\right\|_{Z}^{2}\left\|G_{n}(s)\right\|_{S} \lesssim \varepsilon^{2} s^{-1}\left\|G_{n}(s)\right\|_{S},
$$

and since, by (6.3), $\left\|G_{n}\left(T_{n}\right)\right\|_{S} \lesssim \varepsilon T_{n}^{\delta}$, an application of Gronwall's lemma gives, for $\varepsilon$ small enough,

$$
\left\|G_{n}(s)\right\|_{S} \lesssim \varepsilon s^{\delta}, \quad s \geqslant T_{n}
$$

which, combined with (6.7), provides control of the second and last term in (6.6). We can estimate the $S^{+}$-norm similarly, using Remark 3.8 and the above control, to get

$$
\left\|\partial_{t} G_{n}(s)\right\|_{S^{+}} \lesssim s^{-1} \varepsilon^{2}\left\|G_{n}(s)\right\|_{S^{+}}+\varepsilon^{3} s^{-1+4 \delta}, \quad\left\|G_{n}\left(T_{n}\right)\right\|_{S^{+}} \lesssim \varepsilon T_{n}^{5 \delta} .
$$

This concludes the proof of (6.6).

Now we claim that, for $T_{n} \leqslant t \leqslant T_{n+4}$,

$$
\left\|F(t)-G_{n}(t)\right\|_{S} \lesssim \varepsilon^{3} T_{n}^{-\delta}
$$

Indeed, using (3.1), we see that

$$
\begin{aligned}
F(t)-G_{n}(t)= & i \int_{T_{n}}^{t} \mathcal{E}^{\sigma}[F, F, F] d \sigma \\
& +i \int_{T_{n}}^{t}\left\{\mathcal{R}[F(\sigma), F(\sigma), F(\sigma)]-\mathcal{R}\left[G_{n}(\sigma), G_{n}(\sigma), G_{n}(\sigma)\right]\right\} \frac{d \sigma}{\sigma} .
\end{aligned}
$$

On the one hand, using (6.3) and Proposition 3.1, we obtain that

$$
\left\|\int_{T_{n}}^{t} \mathcal{E}^{\sigma}[F, F, F] d \sigma\right\|_{S} \lesssim \varepsilon^{3} T_{n}^{-2 \delta} .
$$

On the other hand, letting $X(t)=\left\|F(t)-G_{n}(t)\right\|_{Z}$, we see using (7.1) and Lemma 7.4 that

$$
\begin{gathered}
\left\|\int_{T_{n}}^{t}\left\{\mathcal{R}[F(\sigma), F(\sigma), F(\sigma)]-\mathcal{R}\left[G_{n}(\sigma), G_{n}(\sigma), G_{n}(\sigma)\right]\right\} \frac{d \sigma}{\sigma}\right\|_{Z} \\
\lesssim \int_{T_{n}}^{t}\left\{\|F(\sigma)\|_{Z}^{2}+\left\|G_{n}(\sigma)\right\|_{Z}^{2}\right\} X(\sigma) \frac{d \sigma}{\sigma} \lesssim \varepsilon^{2} \int_{T_{n}}^{t} X(\sigma) \frac{d \sigma}{\sigma},
\end{gathered}
$$

so that $X(t)$ is continuous and satisfies

$$
X\left(T_{n}\right)=0, \quad X(t) \lesssim \varepsilon^{3} T_{n}^{-2 \delta}+\varepsilon^{2} \int_{T_{n}}^{t} X(\sigma) \frac{d \sigma}{\sigma} .
$$


An application of Gronwall's lemma gives that $X(t) \lesssim \varepsilon^{3} T_{n}^{-2 \delta}$ for $T_{n} \leqslant t \leqslant T_{n+4}$. We now define $Y(t)=\left\|F(t)-G_{n}(t)\right\|_{S}$. Proceeding as above, we find that $Y\left(T_{n}\right)=0$ and

$$
\begin{aligned}
Y(t) \lesssim & \varepsilon^{3} T_{n}^{-2 \delta}+\varepsilon^{2} \int_{T_{n}}^{t} Y(\sigma) \frac{d \sigma}{\sigma}+\int_{T_{n}}^{t}\left(\|F(\sigma)\|_{Z}\right. \\
& \left.+\left\|G_{n}(\sigma)\right\|_{Z}\right)\left(\|F(\sigma)\|_{S}+\left\|G_{n}(\sigma)\right\|_{S}\right) X(\sigma) \frac{d \sigma}{\sigma} \\
\lesssim & \varepsilon^{3} T_{n}^{-\delta}+\varepsilon^{2} \int_{T_{n}}^{t} Y(\sigma) \frac{d \sigma}{\sigma} .
\end{aligned}
$$

An application of Gronwall's lemma yields (6.8).

We now deduce from this that

$$
\left\|\widetilde{G}_{n}(0)-\widetilde{G}_{n+1}(0)\right\|_{S} \lesssim \varepsilon^{3} e^{-n \delta / 2} .
$$

Indeed, from (6.8), we have that

$$
\left\|\widetilde{G}_{n}(n+1)-\widetilde{G}_{n+1}(n+1)\right\|_{S} \lesssim \varepsilon^{3} e^{-n \delta}, \quad\left\|\widetilde{G}_{n}\right\|_{Z}+\left\|\widetilde{G}_{n+1}\right\|_{Z} \lesssim \varepsilon .
$$

Using Lemma 4.3 (ii), we deduce (6.9) if $\varepsilon$ is small enough. As a consequence, we see that $\left\{\widetilde{G}_{n}(0)\right\}_{n}$ is a Cauchy sequence in $S$ and therefore converges to an element $G_{0, \infty} \in S$ which satisfies that

$$
\left\|G_{0, \infty}\right\|_{Z} \lesssim \varepsilon, \quad\left\|\widetilde{G}_{n}(0)-G_{0, \infty}\right\|_{S} \lesssim \varepsilon^{3} e^{-n \delta / 2} .
$$

Another application of Lemma 4.3 gives

$$
\sup _{\left[0, T_{n+2}\right]}\left\|G_{\infty}(t)-G_{n}(t)\right\|_{S} \lesssim \varepsilon^{3} e^{-n \delta / 4},
$$

where $G_{\infty}(t)=\widetilde{G}_{\infty}(\pi \ln t)$, with $\widetilde{G}_{\infty}$ the solution of (1.3) with initial data $\widetilde{G}_{\infty}(0)=G_{0, \infty}$. We deduce from this and (6.8) that

$$
\begin{aligned}
& \sup _{T_{n} \leqslant t \leqslant T_{n+1}}\left\|G_{\infty}(t)-F(t)\right\|_{S} \\
& \quad \leqslant \sup _{T_{n} \leqslant t \leqslant T_{n+1}}\left\|G_{\infty}(t)-G_{n}(t)\right\|_{S}+\sup _{T_{n} \leqslant t \leqslant T_{n+1}}\left\|G_{n}(t)-F(t)\right\|_{S} \\
& \quad \lesssim \varepsilon^{3} e^{-n \delta / 4} .
\end{aligned}
$$

This finishes the proof. 


\section{Additional estimates}

LEMMA 7.1. Let $R$ be defined as in (4.1). For every sequence $\left(a^{1}\right)_{p},\left(a^{2}\right)_{p},\left(a^{3}\right)_{p}$ indexed by $\mathbb{Z}^{d}, d \leqslant 4$,

$$
\left\|R\left[a^{1}, a^{2}, a^{3}\right]\right\|_{l_{p}^{2}} \leqslant C_{d} \min _{\tau \in \mathfrak{S}_{3}}\left\|a^{\tau(1)}\right\|_{l_{p}^{2}}\left\|a^{\tau(2)}\right\|_{h_{p}^{1}}\left\|a^{\tau(3)}\right\|_{h_{p}^{1}},
$$

and consequently, for any $\sigma \geqslant 0$,

$$
\left\|R\left[a^{1}, a^{2}, a^{3}\right]\right\|_{h_{p}^{\sigma}} \leqslant C_{\sigma, d} \sum_{\tau \in \mathfrak{S}_{3}}\left\|a^{\tau(1)}\right\|_{h_{p}^{\sigma}}\left\|a^{\tau(2)}\right\|_{h_{p}^{1}}\left\|a^{\tau(3)}\right\|_{h_{p}^{1}} .
$$

Proof of Lemma 7.1. One can deduce (7.2) from (7.1) by noting that, pointwise,

$$
\left|p^{\sigma} R\left[a^{1}, a^{2}, a^{3}\right]\right| \lesssim \sum_{\tau \in \mathfrak{S}_{3}} R\left[\left|p^{\sigma} a^{\tau(1)}\right|,\left|a^{\tau(2)}\right|,\left|a^{\tau(3)}\right|\right]
$$

where $p^{\sigma} a$ denotes the sequence $\left(|p|^{\sigma} a_{p}\right)_{p}$ and $|a|$ denotes the sequence $\left(\left|a_{p}\right|\right)_{p}$.

By duality, we need to prove that

$$
\left|\sum_{\substack{p_{0}+p_{2}=p_{1}+p_{3} \\\left|p_{0}\right|^{2}+\left|p_{2}\right|^{2}=\left|p_{1}\right|^{2}+\left|p_{3}\right|^{2}}} a_{p_{0}}^{0} a_{p_{1}}^{1} a_{p_{2}}^{2} a_{p_{3}}^{3}\right| \lesssim\left\|a^{0}\right\|_{l_{p}^{2}} \min _{\tau \in \mathfrak{S}_{3}}\left\|a^{\tau(1)}\right\|_{l_{p}^{2}}\left\|a^{\tau(2)}\right\|_{h_{p}^{1}}\left\|a^{\tau(3)}\right\|_{h_{p}^{1}} .
$$

We will reduce (7.3) to a bound on free solutions on the torus $\mathbb{T}^{d}$. Indeed, if we set

$$
\phi_{j}(y)=\sum_{p \in \mathbb{Z}^{d}} \widetilde{a}_{p}^{j} e^{i p \cdot y}: \mathbb{T}^{d} \rightarrow \mathbb{C}, \quad j=0,1,2,3,
$$

with $\widetilde{a}^{j}=a^{j}$ if $j=1,3$ and $\widetilde{a}_{j}=\overline{a^{j}}$ for $j=0,2$, then we have the identity

$$
\sum_{\substack{p_{0}+p_{2}=p_{1}+p_{3} \\\left|p_{0}\right|^{2}+\left|p_{2}\right|^{2}=\left|p_{1}\right|^{2}+\left|p_{3}\right|^{2}}} a_{p_{0}}^{0} a_{p_{1}}^{1} a_{p_{2}}^{2} a_{p_{3}}^{3}=\int_{\mathbb{T}_{y}^{d} \times \mathbb{T}_{t}} u_{1}(y, t) \overline{u_{2}(y, t)} u_{3}(y, t) \overline{u_{0}(y, t)} d y d t
$$

where $u_{j}(y, t)=e^{i t \Delta_{\mathbb{T}^{d}}}\left(\phi_{j}(y)\right), j=0,1,2,3$. Therefore (7.3) follows from

$$
\left|\int_{\mathbb{T}_{y}^{d} \times \mathbb{T}_{t}} \prod_{j=0}^{3} \tilde{u}_{j}(y, t) d y d t\right| \lesssim\left\|\phi_{0}\right\|_{L_{y}^{2}} \min _{\tau \in \mathfrak{S}_{3}}\left\|\phi_{\tau(1)}\right\|_{L_{y}^{2}}\left\|\phi_{\tau(2)}\right\|_{H_{y}^{1}}\left\|\phi_{\tau(3)}\right\|_{H_{y}^{1}},
$$

where $L_{y}^{2}$ and $H_{y}^{1}$ denote the corresponding Sobolev norms on $\mathbb{T}^{d}$ and $\widetilde{u}_{j} \in$ $\left\{u_{j}, \overline{u_{j}}\right\}$. Estimate (7.4) follows from the analysis in $[4,18,58]$, as we explain 
below. By a slight abuse of notation, inside this proof, we denote again by $P_{N}$ the Littlewood-Paley projector on dyadic scales for functions on the torus $\mathbb{T}^{d}$. By simple renormalization and symmetry arguments (see for example [23, 24]), the estimate (7.4) can be reduced to

$$
\sum_{\substack{N_{0} \leqslant N_{1} \\ N_{3} \leqslant N_{2} \leqslant N_{1}}}\left(N_{2} N_{3}\right)^{-1}\left|\int_{\mathbb{T}^{d+1}} P_{N_{0}} \tilde{u_{0}} P_{N_{1}} \tilde{u_{1}} P_{N_{2}} \tilde{u_{2}} P_{N_{3}} \tilde{u_{3}}\right| \lesssim \prod_{j=0}^{3}\left\|\phi_{j}\right\|_{L_{y}^{2}} .
$$

At this stage, we invoke the classical $L^{4}$ Strichartz estimates by Bourgain [4],

$$
\left\|P_{N} e^{i t \Delta_{\mathbb{T}^{d}}} \phi\right\|_{L_{y, t}^{4}\left(\mathbb{T}^{d+1}\right)} \lesssim N^{s(d)}\|\phi\|_{L_{y}^{2}},
$$

where $s(1)=0, s(d)=((d-2) / 4)+\varepsilon$ for every $\varepsilon>0$ when $d=2,3$, and $s(4)=(d-2) / 4=\frac{1}{2}$ when $d=4$. Using the Galilean invariance of the Schrödinger equation (see for example [57, page 338]), one deduces from (7.6) the bound

$$
\left\|P_{C} e^{i t \Delta_{\mathbb{T}^{d}}} \phi\right\|_{L_{y, t}^{4}\left(\mathbb{T}^{d+1}\right)} \lesssim N^{s(d)}\|\phi\|_{L_{y}^{2}},
$$

where $C$ is a cube of $\mathbb{Z}^{d}$ with side length $N \geqslant 1$ and $P_{C}$ is the corresponding Fourier projector operator. Using (7.7), one gets a bilinear refinement of (7.6),

$$
\left\|\left(P_{N_{1}} e^{i t \Delta_{\mathbb{T}^{d}}} \phi_{1}\right)\left(P_{N_{2}} e^{i t \Delta_{\mathbb{T}^{d}}} \phi_{2}\right)\right\|_{L_{y, t}^{2}\left(\mathbb{T}^{d+1}\right)} \lesssim N_{2}^{2 s(d)}\left\|\phi_{1}\right\|_{L_{y}^{2}}\left\|\phi_{2}\right\|_{L_{y}^{2}},
$$

where $N_{2} \leqslant N_{1}$. Indeed, to get (7.8), it suffices to decompose the dyadic ring of size $N_{1}$ into cubes of size $N_{2}$, to use an orthogonality argument in the spatial variable, and to invoke (7.7). Now, we estimate the left-hand side of (7.5), by using the Cauchy-Schwarz inequality (pairing $P_{N_{0}} u_{0} P_{N_{2}} u_{2}$ and $P_{N_{1}} u_{1} P_{N_{3}} u_{3}$ ) in two ways depending on whether $N_{2} \leqslant N_{0}$ or not and by invoking (7.8), as follows:

$$
\sum_{\substack{N_{0} \sim N_{1} \\ N_{3} \leqslant N_{2} \leqslant N_{0}}}\left(N_{2} N_{3}\right)^{-1}\left(N_{2} N_{3}\right)^{2 s(d)} \prod_{j=0}^{3}\left\|P_{N_{j}} \phi_{j}\right\|_{L_{y}^{2}} .
$$

Since, for $d=1,2,3$, we have $2 s(d)<1$, the expression (7.9) sums properly. This ends the proof for $d=1,2,3$.

For $d=4$, the above argument does not suffice to conclude, because of a lack of summability in $N_{2}$ and $N_{3}$. This causes a significant difficulty, which may be resolved by using the more recent works $[\mathbf{1 8}, \mathbf{5 8}]$, as we now explain. In [18] the four-dimensional estimate (7.6) is improved to

$$
\left\|P_{N} e^{i t \Delta_{\mathbb{T}^{4}}} \phi\right\|_{L_{y, t}^{q}\left(\mathbb{T}^{4+1}\right)} \lesssim N^{2-(6 / q)}\|\phi\|_{L_{y}^{2}}, \quad q>\frac{7}{2} .
$$


Observe that, for $d=4$, the bound (7.6) follows from (7.10) via an interpolation with the elementary $L^{\infty}$ bound

$$
\left\|P_{N} e^{i t \Delta_{\mathbb{T}^{4}}} \phi\right\|_{L_{y, t}^{\infty}\left(\mathbb{T}^{4+1}\right)} \lesssim N^{2}\|\phi\|_{L_{y}^{2}} .
$$

With $(7.10)$ in hand, we can substitute (7.7) by the more refined bound

$$
\left\|P_{C} e^{i t \Delta_{\mathbb{T}^{d}}} \phi\right\|_{L_{y, t}^{4}\left(\mathbb{T}^{4+1}\right)} \lesssim N^{1 / 2}\left(\frac{M}{N}\right)^{\delta}\|\phi\|_{L_{y}^{2}}
$$

for a suitable $\delta>0$, where now $C$ is a 'rectangle' of the form

$$
C=\left\{n \in \mathbb{Z}^{4}:\left|n-n_{0}\right| \leqslant N,\left|a \cdot n-c_{0}\right| \leqslant M\right\}
$$

for some $n_{0}, c_{0} \in \mathbb{R}^{4}$ and $a \in \mathbb{R}^{4},|a|=1$. The proof of (7.12) follows by an interpolation between (7.10) and an $L^{\infty}$ bound of type (7.11) (even though elementary, the $L^{\infty}$ bound is sensitive to the size of $C$, which is crucial for getting the improvement (7.12)). Using (7.12), we may invoke [58, Proposition 2.8], to get the following improvement of (7.8) for $d=4$ :

$$
\left\|\left(P_{N_{1}} e^{i t \Delta_{\mathbb{T}^{4}}} \phi_{1}\right)\left(P_{N_{2}} e^{i t \Delta_{\mathbb{T}^{4}}} \phi_{2}\right)\right\|_{L_{y, t}^{2}\left(\mathbb{T}^{d+1}\right)} \lesssim N_{2}\left(\frac{N_{2}}{N_{1}}+\frac{1}{N_{2}}\right)^{\delta}\left\|\phi_{1}\right\|_{L_{y}^{2}}\left\|\phi_{2}\right\|_{L_{y}^{2}},
$$

for some $\delta>0$, where again $N_{2} \leqslant N_{1}$. Compared to the proof of (7.8), the proof of (7.13) uses an additional almost orthogonality argument in the time variable via an application of (7.12) with $M=\max \left(1, N_{1}^{2} / N_{2}\right)$ (and $N=N_{1}$ ). Using (7.13), we replace (7.9) (for $d=4$ ) by

$$
\sum_{\substack{N_{1} \sim N_{0} \\ N_{3} \leqslant N_{2} \leqslant N_{0}}}\left(\frac{N_{2}}{N_{0}}+\frac{1}{N_{2}}\right)^{\delta}\left(\frac{N_{3}}{N_{1}}+\frac{1}{N_{3}}\right)^{\delta} \prod_{j=0}^{3}\left\|P_{N_{j}} \phi_{j}\right\|_{L_{y}^{2}} .
$$

This expression now sums properly. This completes the proof of Lemma 7.1.

Next, we recall the one-dimensional bilinear Strichartz estimates.

LeMmA 7.2. Assume that $\lambda \geqslant 10 \mu \geqslant 1$ and that $u(t)=e^{i t \partial_{x x}} u_{0}, v(t)=e^{i t \partial_{x x}} v_{0}$. Then, we have the bound

$$
\left\|Q_{\lambda} u \overline{Q_{\mu} v}\right\|_{L_{x, t}^{2}(\mathbb{R} \times \mathbb{R})} \lesssim \lambda^{-1 / 2}\left\|u_{0}\right\|_{L_{x}^{2}(\mathbb{R})}\left\|v_{0}\right\|_{L_{x}^{2}(\mathbb{R})} .
$$

We refer to [28] for the proof of Lemma 7.2 (see also [12] for the earlier higher-dimensional analog of (7.14) and [47] for recent closely related estimates). 
LeMma 7.3. Assume that $N \geqslant 7$. Then we have the bound

$$
\sup _{x \in \mathbb{R}} \sum_{p \in \mathbb{Z}^{d}}\left[1+|p|^{2}\right]\left|e^{i t \partial_{x x}} F_{p}(x)\right|^{2} \lesssim\langle t\rangle^{-1}\left(\|F\|_{Z}^{2}+\langle t\rangle^{-1 / 4}\left(\|x F\|_{L^{2}}^{2}+\|F\|_{H^{N}}^{2}\right)\right) \text {. }
$$

Proof. It suffices to prove the statement for $t \geqslant 1$; for $|t| \leqslant 1$ it simply follows from the Sobolev embedding, and for $t \leqslant-1$ it follows by symmetry. We first claim that there exists a constant $c$ such that

$$
\left|e^{i t \partial_{x x}} f(x)-c \frac{e^{-i\left(x^{2} / 4 t\right)}}{\sqrt{t}} \widehat{f}\left(-\frac{x}{2 t}\right)\right| \lesssim t^{-3 / 4}\|x f\|_{L^{2}} .
$$

Indeed, one can write

$$
\begin{aligned}
e^{i t \partial_{x x}} f(x) & =e^{-i\left(x^{2} / 4 t\right)} \int_{\mathbb{R}} e^{i t \eta^{2}} \widehat{f}\left(\eta-\frac{x}{2 t}\right) d \eta \\
& =e^{-i\left(x^{2} / 4 t\right)}\left(\sum_{l=-1}^{-\infty} I_{l}(x, t)+I(x, t)\right),
\end{aligned}
$$

where

$$
\begin{gathered}
I_{l}(x, t):=\int_{\mathbb{R}} e^{i t \eta^{2}} \phi\left(2^{-l} \eta\right) \widehat{f}\left(\eta-\frac{x}{2 t}\right) d \eta, \\
I(x, t):=\int_{\mathbb{R}} e^{i t \eta^{2}} \tilde{\phi}(\eta) \widehat{f}\left(\eta-\frac{x}{2 t}\right) d \eta,
\end{gathered}
$$

for suitable bump functions $\phi$ and $\tilde{\phi}$ such that the support of $\phi$ does not meet zero. By a crude estimate, we first get that

$$
\left|I_{l}(x, t)-\widehat{f}\left(-\frac{x}{2 t}\right) \int_{\mathbb{R}} e^{i t \eta^{2}} \phi\left(2^{-l} \eta\right) d \eta\right| \lesssim 2^{3 l / 2}\left\|\partial_{\xi} \widehat{f}\right\|_{L^{2}}
$$

On the other hand, an integration by parts gives that

$$
\begin{aligned}
& I_{l}(x, t)-\widehat{f}\left(-\frac{x}{2 t}\right) \int_{\mathbb{R}} e^{i t \eta^{2}} \phi\left(2^{-l} \eta\right) d \eta \\
& \quad=\frac{1}{2 i t} \int_{\mathbb{R}} e^{i t \eta^{2}} \partial_{\eta}\left[\frac{1}{\eta} \phi\left(2^{-l} \eta\right)\left(\widehat{f}\left(\eta-\frac{x}{2 t}\right)-\widehat{f}\left(-\frac{x}{2 t}\right)\right)\right] d \eta .
\end{aligned}
$$

Therefore

$$
\left|I_{l}(x, t)-\widehat{f}\left(-\frac{x}{2 t}\right) \int_{\mathbb{R}} e^{i t \eta^{2}} \phi\left(2^{-l} \eta\right) d \eta\right| \lesssim t^{-1} 2^{-(l / 2)}\left\|\partial_{\xi} \widehat{f}\right\|_{L^{2}}
$$


One also gets a similar bound for $I(x, t)$ (with $l=0)$. Since $\int_{\mathbb{R}} e^{i t \eta^{2}} d \eta=c t^{-1 / 2}$, using (7.17) for $l \leqslant-\frac{1}{2} \log _{2} t$ and (7.18) otherwise, summing over $l$, we recover (7.16).

Now, we deduce that

$$
\begin{aligned}
t \sum_{\substack{p \in \mathbb{Z}^{d} \\
|p| \leqslant t^{1 / 8}}}\left[1+|p|^{2}\right]\left|e^{i t \partial_{x x}} F_{p}(x)\right|^{2} \lesssim & \sum_{p \in \mathbb{Z}^{d}}\left[1+|p|^{2}\right]\left|\widehat{F}_{p}\left(-\frac{x}{2 t}\right)\right|^{2} \\
& +t^{-1 / 2} \sum_{\substack{p \in \mathbb{Z}^{d} \\
|p| \leqslant t^{1 / 8}}}\left[1+|p|^{2}\right]\left\|x F_{p}\right\|_{L^{2}}^{2} .
\end{aligned}
$$

On the other hand, we also have that

$$
\begin{aligned}
t \sum_{|p| \geqslant t^{1 / 8}}\left[1+|p|^{2}\right]\left|e^{i t \partial_{x x}} F_{p}(x)\right|^{2} & \lesssim t^{1-((N-2) / 4)} \sum_{p \in \mathbb{Z}^{d}}\left(1+|p|^{2}\right)^{N-1}\left\|F_{p}\right\|_{H^{1}}^{2} \\
& \lesssim t^{-1 / 4}\|F\|_{H^{N}}^{2}
\end{aligned}
$$

provided that $N \geqslant 7$. This finishes the proof of Lemma 7.3.

We now turn to our basic lemma allowing one to transform suitable $L_{x, y}^{2}$ bounds to bounds in terms of the $L_{x, y}^{2}$-based spaces $S$ and $S^{+}$. We define an $L P$ family $\widetilde{Q}=\left\{\widetilde{Q}_{A}\right\}_{A}$ to be a family of operators (indexed by the dyadic integers) of the form

$$
\widetilde{Q_{1} f}(\xi)=\widetilde{\varphi}(\xi) \widehat{f}(\xi), \quad \widehat{\widetilde{Q}_{A} f}(\xi)=\widetilde{\phi}\left(\frac{\xi}{A}\right) \widehat{f}(\xi), \quad A \geqslant 2
$$

for two smooth functions $\widetilde{\varphi}, \widetilde{\phi} \in C_{c}^{\infty}(\mathbb{R})$, with $\widetilde{\phi} \equiv 0$ in a neighborhood of zero.

We define the set of admissible transformations to be the family of operators $\left\{T_{B}\right\}$ where, for any $B$,

$$
T_{B}=\lambda_{B} \widetilde{Q}_{B}, \quad\left|\lambda_{B}\right| \leqslant 1
$$

for some LP-family $\widetilde{Q}$. Given an trilinear operator $\mathfrak{T}$ and a set $\Lambda$ of 4-tuples of dyadic integers, we define an admissible realization of $\mathfrak{T}$ at $\Lambda$ to be an operator of the form

$$
\mathfrak{T}_{\Lambda}[F, G, H]=\sum_{(A, B, C, D) \in \Lambda} T_{D} \mathfrak{T}\left[T_{A}^{\prime} F, T_{B}^{\prime \prime} G, T_{C}^{\prime \prime \prime} H\right]
$$

for admissible transformations $T, T^{\prime}, T^{\prime \prime}, T^{\prime \prime \prime}$. 
A norm $\mathcal{B}$ is called admissible if, for any admissible transformation $T=$ $\left\{T_{A}\right\}_{A}$, it holds that

$$
\left\|\sum_{A} T_{A} F\right\|_{\mathcal{B}} \lesssim\|F\|_{\mathcal{B}} .
$$

We note that all norms that we consider are admissible.

LEMMA 7.4. Assume that a trilinear operator $\mathfrak{T}$ satisfies

$$
Z \mathfrak{T}[F, G, H]=\mathfrak{T}[Z F, G, H]+\mathfrak{T}[F, Z G, H]+\mathfrak{T}[F, G, Z H],
$$

for $Z \in\left\{x, \partial_{x}, \partial_{y_{1}}, \ldots, \partial_{y_{d}}\right\}$, and let $\Lambda$ be a set of 4-tuples of dyadic integers. With the notation introduced above, assume also that, for all admissible realizations of $\mathfrak{T}$ at $\Lambda$,

$$
\left\|\mathfrak{T}_{\Lambda}\left[F^{a}, F^{b}, F^{c}\right]\right\|_{L^{2}} \leqslant K \min _{\sigma \in \mathfrak{S}_{3}}\left\|F^{\sigma(a)}\right\|_{L^{2}}\left\|F^{\sigma(b)}\right\|_{\mathcal{B}}\left\|F^{\sigma(c)}\right\|_{\mathcal{B}}
$$

for some admissible norm $\mathcal{B}$ such that the Littlewood-Paley projectors $P_{\leqslant M}$ (both in $x$ and in $y$ ) are uniformly bounded on $\mathcal{B}$. Then, for all admissible realizations of $\mathfrak{T}$ at $\Lambda$,

$$
\left\|\mathfrak{T}_{\Lambda}\left[F^{a}, F^{b}, F^{c}\right]\right\|_{S} \lesssim K \max _{\sigma \in \mathfrak{S}_{3}}\left\|F^{\sigma(a)}\right\|_{S}\left\|F^{\sigma(b)}\right\|_{\mathcal{B}}\left\|F^{\sigma(c)}\right\|_{\mathcal{B}}
$$

Assume in addition that, for $Y \in\left\{x,\left(1-\partial_{x x}\right)^{4}\right\}$,

$$
\|Y F\|_{\mathcal{B}} \lesssim \theta_{1}\|F\|_{S^{+}}+\theta_{2}\|F\|_{S} .
$$

Then, for all admissible realizations of $\mathfrak{T}$ at $\Lambda$,

$$
\begin{aligned}
\left\|\mathfrak{T}_{\Lambda}\left[F^{a}, F^{b}, F^{c}\right]\right\|_{S^{+}} \lesssim & K \max _{\sigma \in \mathfrak{S}_{3}}\left\|F^{\sigma(a)}\right\|_{S^{+}}\left(\left\|F^{\sigma(b)}\right\|_{\mathcal{B}}+\theta_{1}\left\|F^{\sigma(b)}\right\|_{S}\right)\left\|F^{\sigma(c)}\right\|_{\mathcal{B}} \\
& +\theta_{2} K \max _{\sigma \in \mathfrak{S}_{3}}\left\|F^{\sigma(a)}\right\|_{S}\left\|F^{\sigma(b)}\right\|_{S}\left\|F^{\sigma(c)}\right\|_{\mathcal{B} .}
\end{aligned}
$$

Proof. The main information we need comes from the computations of the simple commutators

$$
\left[x, \widetilde{Q}_{A}\right]=A^{-1} \widetilde{Q}_{A}^{\prime},
$$

where, if $\widetilde{Q}$ corresponds to the family $(\widetilde{\varphi}, \widetilde{\phi}), \widetilde{Q}^{\prime}$ corresponds to $\left(\widetilde{\varphi}^{\prime}, \widetilde{\phi}^{\prime}\right)$. Clearly (7.25) defines admissible transformations. We may assume that

$$
\left\|F^{a}\right\|_{\mathcal{B}}=\left\|F^{b}\right\|_{\mathcal{B}}=\left\|F^{c}\right\|_{\mathcal{B}}=1, \quad K=1 .
$$

We let $\mathfrak{T}_{\Lambda}$ be an arbitrary admissible realization of $\mathfrak{T}$ at $\Lambda$ (this realization may change from line to line, or even in the same line). For $Z \in\left\{\partial_{x}, \partial_{y_{1}}, \ldots, \partial_{y_{d}}\right\}$, let 
$\mathcal{P}_{v}$ be the projector associated to $|Z|$ (for example $\mathcal{P}_{v}=\phi(|Z| / \nu)$ ). Then we can decompose

$$
\mathcal{P}_{\nu} \mathfrak{T}_{\Lambda}\left[F^{a}, F^{b}, F^{c}\right]=\mathcal{P}_{\nu} \Sigma_{\nu, l o w}+\mathcal{P}_{\nu} \Sigma_{\nu, h i g h},
$$

where

$$
\Sigma_{\nu, \text { low }}:=\mathfrak{T}_{\Lambda}\left[\mathcal{P}_{\leqslant v} F^{a}, \mathcal{P}_{\leqslant v} F^{b}, \mathcal{P}_{\leqslant v} F^{c}\right]
$$

and

$$
\begin{aligned}
\Sigma_{v, \text { high }}:= & \mathfrak{T}_{\Lambda}\left[\mathcal{P}_{\geqslant 2 v} F^{a}, F^{b}, F^{c}\right]+\mathfrak{T}_{\Lambda}\left[\mathcal{P}_{\leqslant v} F^{a}, \mathcal{P}_{\geqslant 2 v} F^{b}, F^{c}\right] \\
& +\mathfrak{T}_{\Lambda}\left[\mathcal{P}_{\leqslant v} F^{a}, \mathcal{P}_{\leqslant v} F^{b}, \mathcal{P}_{\geqslant 2 v} F^{c}\right] .
\end{aligned}
$$

Using the boundedness of $\mathcal{P}_{v}$ on $L^{2}$, we note that, using the Leibniz rule (7.20), for $s$ a positive integer,

$$
\begin{aligned}
\left\|Z^{s} \mathcal{P}_{\nu} \Sigma_{v, \text { low }}\right\|_{L^{2}} & \lesssim v^{-s}\left\|Z^{2 s} \mathcal{P}_{\nu} \Sigma_{\nu, \text { low }}\right\|_{L^{2}} \\
& \lesssim v^{-s} \sum_{\alpha, \beta, \gamma \leqslant v} \sum_{t+u+v \leqslant 2 s} \| \mathfrak{T}_{\Lambda}\left[Z^{t} \mathcal{P}_{\alpha} F^{a}, Z^{u} \mathcal{P}_{\beta} F^{b}, Z^{v} \mathcal{P}_{\gamma} F^{c} \|_{L^{2}}\right.
\end{aligned}
$$

Assume first that $\alpha \geqslant \beta, \gamma$. Using (7.21), and summing over $\beta, \gamma$,

$$
\begin{aligned}
& v^{-s} \sum_{\beta, \gamma \leqslant \alpha \leqslant v} \sum_{t+u+v \leqslant 2 s} \| \mathfrak{T}_{\Lambda}\left[Z^{t} \mathcal{P}_{\alpha} F^{a}, Z^{u} \mathcal{P}_{\beta} F^{b}, Z^{v} \mathcal{P}_{\gamma} F^{c} \|_{L^{2}}\right. \\
& \quad \lesssim \sum_{\alpha \leqslant v}\left(\frac{\alpha}{v}\right)^{s}\left\|\mathcal{P}_{\alpha} Z^{s} F^{a}\right\|_{L^{2}} .
\end{aligned}
$$

The above sum is in $l_{v}^{2}$. We may proceed similarly for the case when $\beta \geqslant \alpha, \gamma$ and the case when $\gamma \geqslant \alpha, \beta$.

To treat $\Sigma_{v, \text { high }}$, we simply use (7.21) to get $\left\|Z^{s} \mathcal{P}_{\nu} \mathfrak{T}_{\Lambda}\left[\mathcal{P}_{\geqslant 2 v} F^{a}, F^{b}, F^{c}\right]\right\|_{L^{2}} \lesssim v^{s}\left\|\mathfrak{T}_{\Lambda}\left[\mathcal{P}_{\geqslant 2 v} F^{a}, F^{b}, F^{c}\right]\right\|_{L^{2}} \lesssim v^{s}\left\|\mathcal{P}_{\geqslant 2 v} F^{a}\right\|_{L^{2}}$, which is in $l_{v}^{2}$, thanks to a standard argument.

This already accounts for most of the components of the $S$-norm, except for the term involving $x$. We first note that

$$
\begin{aligned}
x \mathfrak{T}_{\Lambda}[F, G, H]= & \mathfrak{T}_{\Lambda}[x F, G, H]+\mathfrak{T}_{\Lambda}[F, x G, H]+\mathfrak{T}_{\Lambda}[F, G, x H] \\
& +\sum_{(A, B, C, D) \in \Lambda}\left[x, T_{D}\right] \mathfrak{T}\left[T_{A}^{\prime} F, T_{B}^{\prime \prime} G, T_{C}^{\prime \prime \prime} H\right] \\
& +\sum_{(A, B, C, D) \in \Lambda} T_{D} \mathfrak{T}\left[\left[x, T_{A}^{\prime}\right] F, T_{B}^{\prime \prime} G, T_{C}^{\prime \prime \prime} H\right]
\end{aligned}
$$




$$
\begin{aligned}
& +\sum_{(A, B, C, D) \in \Lambda} T_{D} \mathfrak{T}\left[T_{A}^{\prime} F,\left[x, T_{B}^{\prime \prime}\right] G, T_{C}^{\prime \prime \prime} H\right] \\
& +\sum_{(A, B, C, D) \in \Lambda} T_{D} \mathfrak{T}\left[T_{A}^{\prime} F, T_{B}^{\prime \prime} G,\left[x, T_{C}^{\prime \prime \prime}\right] H\right] .
\end{aligned}
$$

In view of (7.25), we thus see that

$$
\begin{aligned}
x \mathfrak{T}_{\Lambda}[F, G, H]= & \mathfrak{T}_{\Lambda}[x F, G, H]+\mathfrak{T}_{\Lambda}[F, x G, H]+\mathfrak{T}_{\Lambda}[F, G, x H] \\
& +\mathfrak{T}_{\Lambda}[F, G, H] .
\end{aligned}
$$

At this point, we see that all terms in (7.26) are of the form already controlled before. This finishes the proof of (7.22).

Now, from (7.26) and (7.22), we see directly that

$$
\begin{aligned}
\left\|x \mathfrak{T}_{\Lambda}\left[F^{a}, F^{b}, F^{c}\right]\right\|_{S} \lesssim & \sup _{\sigma \in \mathfrak{S}_{3}}\left\|F^{\sigma(a)}\right\|_{S^{+}}\left\|F^{\sigma(b)}\right\|_{B}\left\|F^{\sigma(c)}\right\|_{B} \\
& +\sup _{\sigma \in \mathfrak{S}_{3}}\left\|F^{\sigma(a)}\right\|_{S}\left\|x F^{\sigma(b)}\right\|_{B}\left\|F^{\sigma(c)}\right\|_{B},
\end{aligned}
$$

and, assuming (7.23), we can bound this by the right-hand side of (7.24). The term of the $S^{+}$-norm where $x$ is replaced by $\left(1-\partial_{x x}\right)^{4}$ can be treated similarly to the above analysis. This completes the proof of Lemma 7.4.

We shall also need the following multilinear estimate.

LEMMA 7.5. Let

$$
\frac{1}{p}=\frac{1}{q}+\frac{1}{r}+\frac{1}{s}, \quad 1 \leqslant p, q, r, s \leqslant \infty .
$$

Then

$$
\begin{aligned}
& \left\|\int_{\mathbb{R}^{3}} e^{i x \xi} m(\eta, \kappa) \widehat{f}(\xi-\eta) \widehat{g}(\xi-\eta-\kappa) \widehat{h}(\xi-\kappa) d \eta d \kappa d \xi\right\|_{L^{p}} \\
& \quad \lesssim\left\|\mathcal{F}^{-1} m\right\|_{L^{1}\left(\mathbb{R}^{2}\right)}\|f\|_{L^{q}}\|g\|_{L^{r}}\|h\|_{L^{s}} .
\end{aligned}
$$

The proof of Lemma 7.5 follows from an application of the Parseval identity, the Hölder inequality, and an approximation argument.

\section{Acknowledgements}

The authors want to thank the anonymous referee for carefully reading the manuscript and for the useful suggestions that helped improve the presentation. 
We would also like to thank Michela Procesi and Emanuele Haus for pointing out reference [46].

Z.H. is supported by a Simons Postdoctoral Fellowship and NSF grant DMS1301647, B.P. is partially supported by NSF grant DMS-1142293 and ANR project Scheq ANR-12-JS-0005-01, N.T. is supported by the ERC grant Dispeq., and N.V. is partially supported by project FIRB Dinamiche Dispersive.

\section{References}

[1] T. Alazard and J. M. Delort, 'Global solutions and asymptotic behavior for two dimensional gravity water waves', Preprint, 2013, arXiv:1305.4090.

[2] P. Antonelli, R. Carles and J. D. Silva, 'Scattering for nonlinear Schrödinger equation under partial harmonic confinement', Preprint, 2013, arXiv:1310.1352.

[3] V. Banica, R. Carles and T. Duyckaerts, 'On scattering for NLS: from Euclidean to hyperbolic space', Discrete Contin. Dyn. Syst. 24(4) (2009), 1113-1127.

[4] J. Bourgain, 'Fourier transform restriction phenomena for certain lattice subsets and applications to nonlinear evolution equations. I. Schrödinger equations', Geom. Funct. Anal. 3 (1993), 107-156.

[5] J. Bourgain, 'Exponential sums and nonlinear Schrödinger equations', Geom. Funct. Anal. 3 (1993), 157-178.

[6] J. Bourgain, 'Periodic nonlinear Schrödinger equation and invariant measures', Comm. Math. Phys. 166 (1994), 1-26.

[7] J. Bourgain, 'Aspects of long time behaviour of solutions of nonlinear Hamiltonian evolution equations', Geom. Funct. Anal. 5(2) (1995), 105-140.

[8] J. Bourgain, 'On the growth in time of higher Sobolev norms of smooth solutions of Hamiltonian PDE', Int. Math. Res. Not. IMRN 6 (1996), 277-304.

[9] J. Bourgain, 'Invariant measures for the 2d-defocusing nonlinear Schrödinger equation', Comm. Math. Phys. 176 (1996), 421-445.

[10] J. Bourgain, 'On growth in time of Sobolev norms of smooth solutions of nonlinear Schrödinger equations in $\mathbb{R}^{D}$, J. Anal. Math. 72 (1997), 299-310.

[11] J. Bourgain, 'Quasi-periodic solutions of Hamiltonian perturbations of 2D linear Schrödinger equations', Ann. of Math. (2) 148(2) (1998), 363-439.

[12] J. Bourgain, 'Refinements of Strichartz inequality and applications to 2D-NLS with critical nonlinearity', Int. Math. Res. Not. IMRN (1998), 253-283.

[13] J. Bourgain, 'Global wellposedness of defocusing critical nonlinear Schrödinger equation in the radial case', J. Amer. Math. Soc. 12 (1999), 145-171.

[14] J. Bourgain, 'Growth of Sobolev norms in linear Schrödinger equations with quasi-periodic potential', Comm. Math. Phys. 204(1) (1999), 207-247.

[15] J. Bourgain, 'On growth of Sobolev norms in linear Schrödinger equations with smooth, time-dependent potential', J. Anal. Math. 77 (1999), 315-348.

[16] J. Bourgain, 'Problems in Hamiltonian PDE's', Geom. Funct. Anal. 2000 (2000), 32-56 (special volume, Part I).

[17] J. Bourgain, 'Remarks on stability and diffusion in high-dimensional Hamiltonian systems and partial differential equations', Ergod. Th. \& Dynam. Syst. 24(5) (2004), 1331-1357.

[18] J. Bourgain, 'Moment inequalities for trigonometric polynomials with spectrum in curved hypersurfaces', Israel J. Math. 193(1) (2013), 441-458. 
[19] J. Bourgain and A. Bulut, 'Almost sure global well posedness for the radial nonlinear Schrödinger equation on the unit ball II: the 3D case', J. Eur. Math. Soc. 16 (2014), 1289-1325.

[20] H. Brézis and T. Gallouët, 'Nonlinear Schrödinger evolution equations', Nonlinear Anal. Theory Methods Appl. 4 (1980), 677-681.

[21] N. Burq, P. Gérard and N. Tzvetkov, 'An instability property of the nonlinear Schrödinger equation on $\mathbb{S}^{d}$ ', Math. Res. Lett. 9 (2002), 323-335.

[22] N. Burq, P. Gérard and N. Tzvetkov, 'Strichartz inequalities and the nonlinear Schrödinger equation on compact manifolds', Amer. J. Math. 126 (2004), 569-605.

[23] N. Burq, P. Gérard and N. Tzvetkov, 'Bilinear eigenfunction estimates and the nonlinear Schrödinger equation on surfaces', Invent. Math. 159 (2005), 187-223.

[24] N. Burq, P. Gérard and N. Tzvetkov, 'Multilinear eigenfunction estimates and global existence for the three dimensional nonlinear Schrödinger equations', Ann. Sci. Éc. Norm. Supér. (4) 38 (2005), 255-301.

[25] R. Carles, 'Geometric optics and long range scattering for one-dimensional nonlinear Schrödinger equations', Commun. Math. Phys. 220(1) (2001), 41-67.

[26] R. Carles and E. Faou, 'Energy cascade for NLS on the torus', Discrete Contin. Dyn. Syst. 32(6) (2012), 2063-2077.

[27] T. Cazenave, Semilinear Schrödinger Equations, Courant Lecture Notes in Mathematics, 10 (New York University, Courant Institute of Mathematical Sciences, New York; American Mathematical Society, Providence, RI, 2003).

[28] J. Colliander, M. Keel, G. Staffilani, H. Takaoka and T. Tao, 'Global well-posedness for Schrödinger equations with derivative', SIAM J. Math. Anal. 33 (2001), 649-669.

[29] J. Colliander, M. Keel, G. Staffilani, H. Takaoka and T. Tao, 'Global well-posedness and scattering for the energy-critical nonlinear Schrödinger equation in $\mathbb{R}^{3}$, Ann. of Math. (2) 167 (2008), 767-865.

[30] J. Colliander, M. Keel, G. Staffilani, H. Takaoka and T. Tao, 'Transfer of energy to high frequencies in the cubic defocusing nonlinear Schrödinger equation', Invent. Math. 181(1) (2010), 39-113.

[31] J. Colliander, S. Kwon and T. Oh, 'A remark on normal forms and the 'upside-down' I-method for periodic NLS: growth of higher Sobolev norms', J. Anal. Math 118(1) (2012), 55-82.

[32] P. Deift and X. Zhou, 'Long-time asymptotics for solutions of the NLS equation with initial data in a weighted Sobolev space, Dedicated to the memory of Jürgen K. Moser', Comm. Pure Appl. Math. 56(8) (2003), 1029-1077.

[33] J.-M. Delort, 'Existence globale et comportement asymptotique pour l'équation de KleinGordon quasi linéaire à données petites en dimension 1', Ann. Sci. Éc. Norm. Supér. (4) 34(1) (2001), 1-61.

[34] B. Dodson, 'Global well-posedness and scattering for the defocusing, $L^{2}$-critical, nonlinear Schrdinger equation when $d=1$ ', Preprint, 2010, arXiv:1010.0040.

[35] B. Dodson, 'Global well-posedness and scattering for the defocusing, $L^{2}$-critical, nonlinear Schrödinger equation when $d=2$ ', Preprint, 2010, arXiv:1006.1375.

[36] B. Dodson, 'Global well-posedness and scattering for the defocusing, energy-critical, nonlinear Schrödinger equation in the exterior of a convex obstacle when $d=4$ ', Preprint, 2011, arXiv:1112.0710.

[37] L. H. Eliasson and S. Kuksin, 'KAM for the nonlinear Schrödinger equation', Ann. of Math. (2) 172(1) (2010), 371-435. 
[38] E. Faou, P. Germain and Z. Hani, 'The weakly nonlinear large box limit of the 2D cubic nonlinear Schrödinger equation', J. Amer. Math. Soc. (JAMS), to appear, Preprint, 2013, arXiv:1308.6267.

[39] P. Gérard and S. Grellier, 'The Szegö cubic equation', Ann. Sci. Éc. Norm. Supér. (4) 43 (2010), 761-809.

[40] P. Gérard and S. Grellier, 'An explicit formula for the cubic Szegö equation', Trans. Amer. Math. Soc. 367 (2015), 2979-2995.

[41] P. Gérard and S. Grellier, 'Effective integrable dynamics for some nonlinear wave equation', Anal. PDE 5 (2012), 1139-1155.

[42] P. Germain, N. Masmoudi and J. Shatah, 'Global solutions for 3D quadratic Schrödinger equations', Int. Math. Res. Not. IMRN (2009), 414-432.

[43] P. Germain, N. Masmoudi and J. Shatah, 'Global solutions for the gravity water waves equation in dimension 3', Ann. of Math. (2) 175 (2012), 691-754.

[44] B. Grebert, E. Paturel and L. Thomann, 'Beating effects in cubic Schrödinger systems and growth of Sobolev norms', Nonlinearity 26 (2013), 1361-1376.

[45] M. Guardia and V. Kaloshin, 'Growth of Sobolev norms in the cubic defocusing nonlinear Schrödinger equation', J. Eur. Math. Soc. 17(1) (2015), 71-149.

[46] M. Guardia and V. Kaloshin, 'Erratum to "Growth of Sobolev norms in the cubic defocusing nonlinear Schrödinger equation", personal communication.

[47] S. Guo, 'On the 1D cubic NLS in an almost critical space', Master Thesis, University of Bonn (2012).

[48] Y. Guo, A. Ionescu and B. Pausader, 'The Euler-Maxwell 2 fluid in 3D', Preprint, 2013, arXiv:1303.1060.

[49] Z. Hani, 'Global well-posedness of the cubic nonlinear Schrödinger equation on compact manifolds without boundary', Comm. Partial Differential Equations 37(7) (2012), 1186-1236.

[50] Z. Hani, 'Long-time strong instability and unbounded orbits for some periodic nonlinear Schödinger equations', Arch. Ration. Mech. Anal. 211(3) (2014), 929-964.

[51] Z. Hani and B. Pausader, 'On scattering for the quintic defocusing nonlinear Schrödinger equation on $\mathbb{R} \times \mathbb{T}^{2}$, Comm. Pure Appl. Math. 67(9) (2014), 1466-1542.

[52] Z. Hani and L. Thomann, 'Asymptotic behavior of the nonlinear Schrödinger equation with harmonic trapping', Commun. Pure Appl. Math. (CPAM) Preprint, 2014, arXiv:1408.6213, published online: 15 July 2015, doi:10.1002/cpa.21594.

[53] N. Hayashi, C. Li and P. Naumkin, 'Modified wave operator for a system of nonlinear Schrödinger equations in 2d', Comm. Partial Differential Equations 37(6) (2012), 947-968.

[54] N. Hayashi and P. Naumkin, 'Asymptotics for large time of solutions to the nonlinear Schrödinger and Hartree equations', Amer. J. Math. 120(2) (1998), 369-389.

[55] N. Hayashi, P. Naumkin, A. Shimomura and S. Tonegawa, 'Modified wave operators for nonlinear Schrödinger equations in one and two dimensions', Electron. J. Differential Equations 62 (2004), 16 pp.

[56] S. Herr, 'The quintic nonlinear Schrödinger equation on three-dimensional Zoll manifolds', Amer. J. Math. 135(5) (2013), 1271-1290.

[57] S. Herr, D. Tataru and N. Tzvetkov, 'Global well-posedness of the energy critical nonlinear Schrödinger equation with small initial data in $H^{1}\left(\mathbb{T}^{3}\right)$ ', Duke Math. J. 159 (2011), 329-349.

[58] S. Herr, D. Tataru and N. Tzvetkov, 'Strichartz estimates for partially periodic solutions to Schrödinger equations in $4 d$ and applications', J. Ang. Math. 2014(690) (2012), 65-78. 
[59] A. D. Ionescu and B. Pausader, 'Global wellposedness of the energy-critical defocusing NLS on $\mathbb{R} \times \mathbb{T}^{3}$, Commun. Math. Phys. 312(3) (2012), 781-831.

[60] A. D. Ionescu and B. Pausader, 'The energy-critical defocusing NLS on $\mathbb{T}^{3}$, Duke Math. J. 161(8) (2012), 1581-1612.

[61] A. D. Ionescu, B. Pausader and G. Staffilani, 'On the global well-posedness of energycritical Schrödinger equations in curved spaces', Anal. PDE 5(4) (2012), 705-746.

[62] A. Ionescu and B. Pausader, 'Global solutions of quasilinear systems of Klein-Gordon equations in 3D', J. Eur. Math. Soc. 16(11) (2014), 2355-2431.

[63] A. D. Ionescu and F. Pusateri, 'Nonlinear fractional Schrödinger equations in one dimensions', J. Funct. Anal. 266(1) (2014), 139-176.

[64] A. D. Ionescu and F. Pusateri, 'Global solutions for the gravity water waves system in 2d', Invent. Math. 199(3) (2015), 653-804.

[65] A. D. Ionescu and G. Staffilani, 'Semilinear Schrödinger flows on hyperbolic spaces: scattering in $H^{1}$, Math. Ann. 345 (2009), 133-158.

[66] J. Kato and F. Pusateri, 'A new proof of long range scattering for critical nonlinear Schrödinger equations', J. Diff. Int. Equ. 24(9-10) (2011).

[67] C. E. Kenig and F. Merle, 'Global well-posedness, scattering and blow-up for the energycritical, focusing, nonlinear Schrödinger equation in the radial case', Invent. Math. 166 (2006), 645-675.

[68] R. Killip, T. Tao and M. Visan, 'The cubic nonlinear Schrödinger equation in two dimensions with radial data', J. Eur. Math. Soc. 11 (2009), 1203-1258.

[69] R. Killip and M. Visan, 'Global well-posedness and scattering for the defocusing quintic NLS in three dimensions', Anal. PDE 5 (2012), 855-885.

[70] R. Killip, M. Visan and X. Zhang, 'Quintic NLS in the exterior of a strictly convex obstacle', Preprint, 2012, arXiv:1208.4904.

[71] S. Kuksin, 'Oscillations in space-periodic nonlinear Schrödinger equations', Geom. Funct. Anal. 7(2) (1997), 338-363.

[72] S. Kuksin and J. Pöschel, 'Invariant Cantor manifolds of quasi periodic oscillations for a nonlinear Schrödinger equation', Ann. of Math. (2) 143 (1996), 149-179.

[73] A. Majda, D. McLaughlin and E. Tabak, 'A one-dimensional model for dispersive wave turbulence', J. Nonlinear Sci. 7(1) (1997), 9-44.

[74] T. Ozawa, 'Long range scattering for nonlinear Schrödinger equations in one space dimension', Commun. Math. Phys. 139 (1991), 479-493.

[75] B. Pausader, N. Tzvetkov and X. Wang, 'Global regularity for the energy-critical NLS on $\mathbb{S}^{3}$, Ann. Inst. H. Poincaré Anal. Non Linéaire 31(2) (2014), 315-338.

[76] O. Pocovnicu, 'Explicit formula for the solution of the Szegö equation on the real line and applications', Discrete Contin. Dyn. Syst. 31 (2011), 607-649.

[77] O. Pocovnicu, 'First and second order approximations for a nonlinear wave equation', J. Dynam. Differential Equations 25(2) (2013), 305-333. 29.

[78] M. Procesi and C. Procesi, 'A KAM algorithm for the resonant nonlinear Schrödinger equation', Preprint arXiv:1211.4242.

[79] E. Ryckman and M. Visan, 'Global well-posedness and scattering for the defocusing energycritical nonlinear Schrödinger equation in $\mathbb{R}^{1+4}$, Amer. J. Math. 129 (2007), 1-60.

[80] V. Sohinger, 'Bounds on the growth of high Sobolev norms of solutions to nonlinear Schrödinger equations on $S^{1}$, Differential Integral Equations 24(7-8) (2011), 653-718.

[81] G. Staffilani, 'On the growth of high Sobolev norms of solutions for KdV and Schrödinger equations', Duke Math. J. 86(1) (1997), 109-142. 
[82] H. Takaoka and N. Tzvetkov, 'On $2 D$ Nonlinear Schrödinger equations with data on $\mathbb{R} \times \mathbb{T}$ ', J. Funct. Anal. 182 (2001), 427-442.

[83] S. Terracini, N. Tzvetkov and N. Visciglia, 'The NLS ground states on product spaces', Anal. PDE 7(1) (2014), 73-96.

[84] N. Tzvetkov, 'Invariant measures for the defocusing NLS', Ann. Inst. Fourier 58 (2008), 2543-2604.

[85] N. Tzvetkov and N. Visciglia, 'Small data scattering for the nonlinear Schrödinger equation on product spaces', Comm. Partial Differential Equations 37(1) (2012), 125-135.

[86] N. Tzvetkov and N. Visciglia, 'Well-posedness and scattering for NLS on $\mathbb{R}^{d} \times \mathbb{T}$ in the energy space', Preprint, 2014, arXiv:1409.3938.

[87] M. Visan, 'Global well-posedness and scattering for the defocusing cubic NLS in four dimensions', Int. Math. Res. Not. IMRN 2011 (2011), doi:10.1093/imrn/rnr051.

[88] H. Xu, 'Large time blow up for a perturbation of the cubic Szegö equation', Preprint, 2013, arXiv:1307.5284.

[89] V. E. Zakharov and A. B. Shabat, 'Exact theory of two-dimensional self-focusing and onedimensional self-modulation of waves in nonlinear media', Sov. Phys. JEPT 34 (1972), 62-69.

[90] V. E. Zakharov, V. L'vov and G. Falkovich, Kolmogorov Spectra of Turbulence 1. Wave Turbulence, Springer Series in Nonlinear Dynamics, (Springer, Berlin, Heidelberg, 1992). 